\title{
A comparative review of peridynamics and phase-field models for engineering fracture mechanics
}

\author{
Patrick Diehl $^{1}$ (D) $\cdot$ Robert Lipton $^{2}$ (D) $\cdot$ Thomas Wick $^{3,4}$ (D) Mayank Tyagi $^{5}$ (D)
}

Received: 13 July 2021 / Accepted: 20 January 2022 / Published online: 18 February 2022

(c) The Author(s) 2022

\begin{abstract}
Computational modeling of the initiation and propagation of complex fracture is central to the discipline of engineering fracture mechanics. This review focuses on two promising approaches: phase-field (PF) and peridynamic (PD) models applied to this class of problems. The basic concepts consisting of constitutive models, failure criteria, discretization schemes, and numerical analysis are briefly summarized for both models. Validation against experimental data is essential for all computational methods to demonstrate predictive accuracy. To that end, the Sandia Fracture Challenge and similar experimental data sets where both models could be benchmarked against are showcased. Emphasis is made to converge on common metrics for the evaluation of these two fracture modeling approaches. Both PD and PF models are assessed in terms of their computational effort and predictive capabilities, with their relative advantages and challenges are summarized.
\end{abstract}

Keywords Peridynamics · Phase-field · Validation studies

Thomas Wick

thomas.wick@ifam.uni-hannover.de

Patrick Diehl

pdiehl@cct.lsu.edu

Robert Lipton

lipton@1su.edu

Mayank Tyagi

mtyagi@1su.edu

1 Center of Computation \& Technology, Louisiana State University, Baton Rouge, LA 70803, USA

2 Department of Mathematics, Center of Computation \& Technology, Louisiana State University, Baton Rouge, LA 70808, USA

3 Institut für Angewandte Mathematik, Leibniz Universität Hannover, Welfengarten 1, 30167 Hannover, Germany

4 Université Paris-Saclay, ENS Paris-Saclay, LMT Laboratoire de Mécanique et Technologie, 91190 Gif-sur-Yvette, France

5 Craft \& Hawkins Department of Petroleum Engineering, Center for Computation \& Technology, Louisiana State University, Baton Rouge, LA 70803, USA

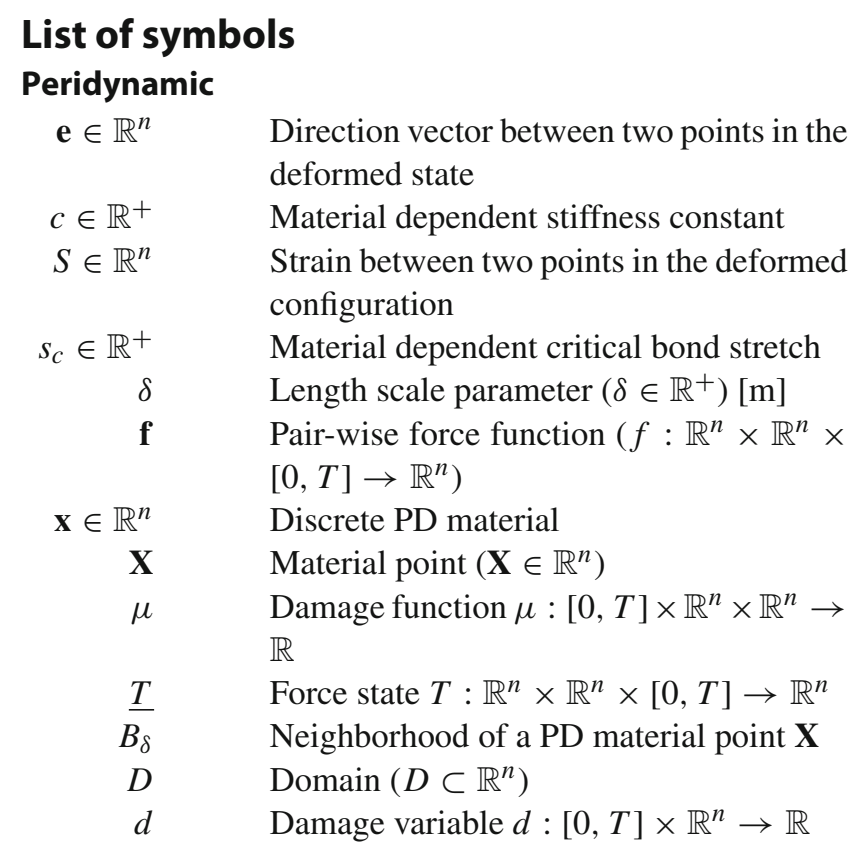

Phase-field

$\Gamma \quad$ Crack set $\left(\Gamma \subset \mathbb{R}^{n-1}\right)$

$\gamma \quad$ Crack surface density function

$\kappa \quad$ Regularization parameter $\left(\kappa \in \mathbb{R}^{+}\right)$

b $^{*} \quad$ Body force $\left[\mathrm{N} / \mathrm{m}^{3}\right]$

$\mathbf{t}^{*} \quad$ Boundary traction $[\mathrm{Pa} / \mathrm{m}]$ 


$\begin{aligned} \Omega & \text { Domain }\left(\Omega \subset \mathbb{R}^{n}\right) \\ \partial \Omega & \text { Boundary }\left(\partial \Omega \subset \mathbb{R}^{n-1}\right) \\ \partial \Omega_{t} & \text { Traction boundary }\left(\partial \Omega_{t} \subset \mathbb{R}^{n-1}\right) \\ \partial \Omega_{u} & \text { Displacement boundary }\left(\partial \Omega_{u} \subset \mathbb{R}^{n-1}\right) \\ \partial_{t} \varphi \leq 0 & \text { Crack irreversibility constraint } \\ \varphi & \text { Phase-field crack function }(\varphi \in[0,1]) \\ \psi_{c} & \text { Surface energy } \\ \psi_{s} & \text { Strain energy } \\ E & \text { Energy [J] } \\ F & \text { External energy potential }[\mathrm{J}] \\ g(\varphi) & \text { Degradation function } \\ l_{0} & \text { Length scale parameter }\left(l_{0} \in \mathbb{R}^{+}\right)[\mathrm{m}] \\ P & \text { Potential energy }[\mathrm{J}]\end{aligned}$

Other symbols

$\begin{aligned} \ddot{\mathbf{u}} & \text { Acceleration }\left(\ddot{\mathbf{u}} \in \mathbb{R}^{n}\right)\left[\mathrm{m} / \mathrm{s}^{2}\right] \\ \mathbf{u} & \text { Displacement }\left(\mathbf{u} \in \mathbb{R}^{n}\right)[\mathrm{m}] \\ \varepsilon(\mathbf{u}) & \text { Linearized strain tensor } \\ \varrho & \text { Material's density }\left[\mathrm{kg} / \mathrm{m}^{3}\right] \\ G_{c} & \text { Critical energy release rate }\left[\mathrm{J} \mathrm{m}^{-2}\right] \\ n & \text { Dimension } n=\{1,2,3\} \\ T & \text { Final Time }[\mathrm{s}] \\ t & \text { Current time }[\mathrm{s}]\end{aligned}$

\section{Introduction}

Fracturing phenomena in natural and engineered systems is studied extensively experimentally, theoretically, and computationally. Here we focus on two promising approaches: phase-field (PF) and peridynamics (PD) for the computational modeling of fractures in materials. Both approaches are distinct from earlier ones as they seek to predict crack path as functions of specimen loading and geometry. Ideally, these approaches attempt the computational predictions after an initial calibration using engineering constants such as Young's modulus and fracture toughness specific to the material. These ambitious approaches are called free fracture models. This review is intended as a snapshot capturing in broad strokes the modeling details, assumptions, experimental data sets, and numerical simulations necessary for validation. These methods have the potential to address fundamental issues in complex fracturing with minimal introduction of phenomenological modeling assumptions and numerical tuning parameters. However, systematic comparative analysis for these models, together with validation studies on the set of experiments, are rare. In this review, we attempt to initiate such a comparative analysis and, when possible, invoke validation studies from the experimental literature.

As an example of an engineering fracture mechanics application, Hattori et al. (2017) [1] presented a comprehensive comparison of various numerical approaches for the hydraulic fracturing of shale and showed the advantages as well as limitations of many numerical approaches including peridynamics (PD) and phase-field (PF). However, this comparative analysis for various models lacked validation studies on the same set of hydraulic fracturing experiments in order to evaluate predictive capabilities of numerical models. Our review is motivated by the recent workshops on phasefield, peridynamics, and experimental fracture mechanics held at The Banff International Research Station: Hydraulic Fracturing: Modeling, Simulation, and Experiment ${ }^{1}$, and the Workshop on Experimental and Computational Fracture Mechanics $^{2}[2]$.

\subsection{Other review papers}

One of the first overview papers of peridynamic and nonlocal modeling was written by Du and Lipton ${ }^{3}$ in the SIAM news, volume 47 (2014). However, this article was quite brief. In 2019, Javili et al. [3] published a review emphasizing the applications of PD. In the same year Diehl et al. [4] published a review with the focus on benchmarking PD against experimental data. In 2021, Isiet et al. [5] published a review on the usage of PD for impact damage. In the same year, Hattori et al. [6] published a review on the usage of PD in reinforced concrete structures. Zhou and Wang [7] published a review on the usage of PD in geomaterials in 2021. To summarize, except for the first PD review in 2019, all reviews focused on some specific topic. Hidayat et al. [8] published a review on the connection of meshfree PD between other meshfree methods in 2021. In addition, the following three books about PD are available [9-11].

In what follows, we now list PF review papers and monographs. The first summary on variational modeling of fracture was by the founding authors Bourdin, Francfort and Marigo [12]. Although they explicitly state on page 7 that they are not attempting to review and access existing literature, their work describes on 148 pages the current state-of-the-art in the year 2008. The first review papers (that have explicitly this purpose) with regard to computational/engineering aspects of phase-field (variational) fracture were published by Rabczuk [13] and Ambati, Gerasimov, and De Lorenzis in 2015 [14]. In the latter study, despite review aspects, a new formulation for stress splitting is proposed therein. Due to the ongoing increasing popularity, shortly after, various other reviews, monographs, and news articles appeared. A short SIAM news article about the latest developments and future perspectives was published by Bourdin and

\footnotetext{
${ }^{1}$ https://www.birs.ca/events/2018/5-day-workshops/18w5085.

2 http://wfm2020.usacm.org/.

3 https://sinews.siam.org/Details-Page/peridynamics-fracture-andnonlocal-continuum-models.
} 
Francfort in 2019 [15]. An extensive review paper on theoretical and computational aspects was done by $\mathrm{Wu}$ et al. in the year 2020 [16]. The authors also discuss the success or failures of several benchmark problems for quasi-static, dynamic brittle/cohesive fracture. Only considering phase-field (variational) fracture, this review paper certainly exceeds in topics, modeling details and numerical aspects and examples of our current work. In the same year, a monograph on multiphysics phase-field fracture was published by Wick [17]. Therein, the focus is briefly on modeling of classical variational phasefield fracture, error estimation and adaptivity, and including other physics phenomena via volume or interface coupling techniques. Most recently in 2021, one of the original authors, namely, Francfort, published an article entitled with 'Variational fracture: Twenty years after' [18].

\subsection{Purpose and value of this review paper}

In view of existing (recent) reviews on both approaches, we shall explain the purpose and value of the current review paper. First and most importantly, both PD and $\mathrm{PF}$ are appearing to be the most prominent approaches for free fracture modeling. Other notable approaches are the displacement-discontinuity method [19], cohesive-zone models [20], boundary elements [21], XFEM/GFEM [2224], and the eigen-erosion framework [25-28]. A comparative review between XFEM (extended finite elements), mixed FEM, and phase-field models appeared in [29].

The present paper provides for the first time a comparison between both approaches (PD and PF). For this reason, the mathematical descriptions remain rather short while focusing on the key ingredients that allow for comparison of PD and PF. It is through this lens we reference the existing models and numerical methods from the literature. The Sandia Fracture Challenge is chosen for validation against experimental data. The first part of the challenge is to calibrate a free fracture model to simple prototypical problems, e. g. a tensile test, and use this calibration to simulate the crack and fracture phenomena. Thus, the model parameters can not be fitted to the complex scenario and have been instead calibrated using the simple scenario. This additional step of calibration makes the Sandia Fracture Challenge an excellent problem to benchmark phase-field models and peridynamic models to assess their performance on different kinds of crack and fracture phenomena. These numerical, computational, and experimental side-by-side comparisons allow us to identify similarities, common challenges, and specific aspects to each method.

\subsection{Outline}

The paper is structured as follows: Sect. 2 introduces the two models and provides a basis on which the models can be compared and contrasted. This summary is an adaptation and extension of the review papers and monograph $[3,4,16,17]$. Section 3 addresses the fracture physics perspective from the macroscale view. Section 4 attempts to compare the predictive accuracy of the two models for validation against the experimental data. To that end, the Sandia Fracture Challenges data sets were analyzed and computed, the $R^{2}$ correlation of relative errors between the simulations and the experiment are presented. Section 5 compares computational aspects of the two models as well as pointing out challenges and opportunities for development. Finally, Sect. 6 summarizes the modeling capabilities of PD and PF.

\section{Overview of models and numerical methodology}

This section briefly introduces the two methods, peridynamics (PD) and phase field (PF), respectively. A brief overview of the governing equations, material models, discretizations, numerical analysis, and advanced visualization methods is given. We introduce the ingredients for the comparison of these two models and provide references to the extended literature for the interested reader.

\subsection{The governing equation of peridynamics}

Peridynamics (PD), is a non-local adaptation of classical continuum mechanics (CCM). In PD, each material point $\mathbf{X}$ interacts with its neighbors inside a finite interaction zone $B_{\delta}(\mathbf{X})$ with the length $\delta$, see Fig. 1. This type of non-local interaction principle is also seen in molecular dynamics (MD) [30] and smoothed particle hydrodynamics (SPH) [31,32] simulations. The important feature of PD fracture modeling is that the interaction between the intact

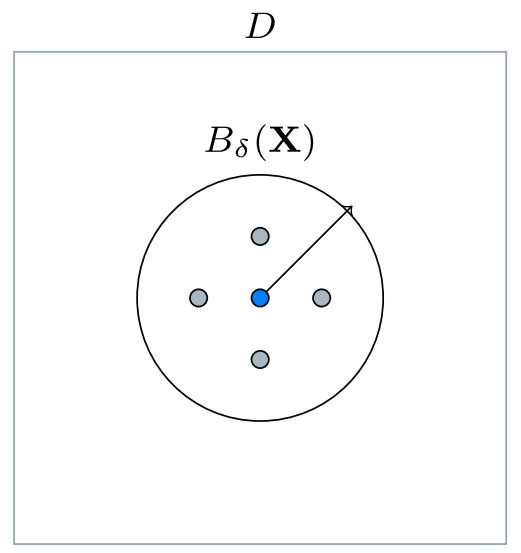

Fig. 1 Sketch for the principle of peridynamics where a material point $X$ interacts with its neighbors inside a finite interaction zone $B_{\delta}(\mathbf{X})$ with the length $\delta$ 
material and fractured material is modeled implicitly through a non-local field equation that remains the same everywhere in the computational domain. Material damage and fracture phenomena are captured through a force versus strain constitutive model. Because of this, PD is able to capture fracture as an emergent phenomena arising from the non-local equation of motion. This contrasts with classic fracture theory where, off the crack, the elastic interaction is modeled by the equation of elastodynamics and the fracture set is a free boundary with motion coupled to elastodynamics through a physically motivated kinetic relation. Other recently developed non-local models exhibiting emergent behavior include the Cucker Smail equation, where swarming behavior emerges from leaderless flocks of birds [33-36].

The equation of motion for bond-based peridynamics proposed by Silling $[37,38]$ reads as

$$
\varrho(\mathbf{X}) \ddot{\mathbf{u}}(t, \mathbf{X})=\int_{B_{\delta}(\mathbf{X})} \mathbf{f}\left(\mathbf{u}\left(t, \mathbf{X}^{\prime}\right)-\mathbf{u}(t, \mathbf{X}), \mathbf{X}^{\prime}-\mathbf{X}\right) d \mathbf{X}^{\prime}+\mathbf{b}(t, \mathbf{X}),
$$

where $\varrho \in \mathbb{R}$ is the material density $\ddot{\mathbf{u}} \in \mathbb{R}^{n}$ is the acceleration at time $t \in \mathbb{R}$ of the material point $\mathbf{X} \in \mathbb{R}^{n}$, f : $\mathbb{R}^{n} \times \mathbb{R}^{n} \times[0, T] \rightarrow \mathbb{R}^{n}$ is the pair-wise force function, $\mathbf{b} \in \mathbb{R}^{n}$ is an external force density, and $\mathbf{u} \in \mathbb{R}^{n}$ is the state of deformation at a point in space time, $(t, \mathbf{X})$. The constitutive model for the pair-wise force function $\mathbf{f}$ is material dependent and defines how the internal forces react to the displacement $\mathbf{u}$. For the elastic regime the force interaction between a pair of points $\mathbf{X}$ and $\mathbf{X}^{\prime}$ is a so-called bond and contributes to the name of bond-based PD. The pair-wise interaction leads to a constraint on the Poisson ratio $[39,40]$.

To overcome the Poisson ratio constraint more general non-local interactions are included in the "state-based" peridynamic model. The generic state-based peridynamic equation of motion introduced by Silling et al. [41] reads as

$\varrho(\mathbf{X}) \ddot{\mathbf{u}}(t, \mathbf{X})=\int_{B_{\delta}(\mathbf{X})}\left(\underline{T}[\mathbf{X}, t]\left\langle\mathbf{X}^{\prime}-\mathbf{X}\right\rangle-\underline{T}\left[\mathbf{X}^{\prime}, t\right]\left\langle\mathbf{X}-\mathbf{X}^{\prime}\right\rangle\right) d \mathbf{X}^{\prime}+\mathbf{b}(t, \mathbf{X})$,

where the pair-wise force function $\mathbf{f}$ is exchanged with the so-called peridynamic force state $\underline{T}: \mathbb{R}^{n} \times \mathbb{R}^{n} \times[0, T] \rightarrow$ $\mathbb{R}^{n}$. The usage of PD states lead to the name state-based PD. A peridynamic state relates to second order tensor and they assign each point in a neighborhood a tensorial quantity. However, in general, it is not a linear or continuous function with respect to $\mathbf{X}-\mathbf{X}^{\prime}$. For more details, we refer to [41, Section 2].

In peridynamics, material damage is part of the constitutive modeling and the material is viewed as damaged when the force state at a point $\mathbf{X}$ no longer influences a material point $\mathbf{X}^{\prime}$ and vice versa. Damage occurs when the difference between deformation states at each point $\mathbf{X}$ and $\mathbf{X}^{\prime}$ surpass a threshold. The specifics of how this occurs depends on the material model used. For example, for pairwise force functions $\mathbf{f}$ the force acting between two points is often referred to as a bond. When the pairwise force is zero, it is said that the bond is broken. Bonds can break irreversibly, or alternatively they can heal under the right conditions, this depends upon the material model used.

To fix ideas, we illustrate the constitutive law given by the prototype brittle microelastic (PMB) model introduced in [38]. The deformation of a point $\mathbf{X}$ is $\mathbf{u}(t, \mathbf{X})$ and its position in the deformed configuration is $\mathbf{X}+\mathbf{u}(t, \mathbf{X})$. In this model, the pair-wise force function depends on the strain between two points in the deformed configuration given by

$$
\begin{aligned}
& S\left(t, \mathbf{X}, \mathbf{X}^{\prime}\right) \\
& \quad:=\frac{\left\|\left(\mathbf{X}^{\prime}+\mathbf{u}\left(t, \mathbf{X}^{\prime}\right)\right)-(\mathbf{X}+\mathbf{u}(t, \mathbf{X}))\right\|-\left\|\mathbf{X}^{\prime}-\mathbf{X}\right\|}{\left\|\mathbf{X}^{\prime}-\mathbf{X}\right\|} .
\end{aligned}
$$

The direction vector between two points in the deformed configuration is given by

$\mathbf{e}=\frac{\left(\mathbf{X}^{\prime}+\mathbf{u}\left(t, \mathbf{X}^{\prime}\right)\right)-(\mathbf{X}+\mathbf{u}(t, \mathbf{X}))}{\left\|\left(\mathbf{X}^{\prime}+\mathbf{u}\left(t, \mathbf{X}^{\prime}\right)\right)-(\mathbf{X}+\mathbf{u}(t, \mathbf{X}))\right\|}$.

The force directed between the two points in the deformed configuration is given by the constitutive law

$\mathbf{f}\left(\mathbf{u}\left(t, \mathbf{X}^{\prime}\right)-\mathbf{u}(t, \mathbf{X}), \mathbf{X}^{\prime}-\mathbf{X}\right):=$

$\mu\left(t, \mathbf{X}, \mathbf{X}^{\prime}\right) c S\left(t, \mathbf{X}, \mathbf{X}^{\prime}\right) \mathbf{e}$,

where $c>0$ is the material dependent stiffness constant, and the scalar damage function $\mu$ is given by

$\mu\left(t, \mathbf{X}, \mathbf{X}^{\prime}\right):= \begin{cases}1, & S\left(t, \mathbf{X}, \mathbf{X}^{\prime}\right) \leq s_{c} \\ 0, & \text { otherwise }\end{cases}$

where $s_{c}>0$ is the material dependent critical bond stretch. Once the force between the two points goes to zero it stays zero. It is noted that there are several other definitions for the damage function $\mu$ using strain-based criteria $[38,42]$, as well as stress-based [43], or energy-based damage criteria [44,45], see Fig. 4.

In numerical implementations crack path evolution is recovered as a post-processing step after simulation. To identify the crack we introduce the damage field $d:[0, T] \times$ $\mathbb{R}^{n} \rightarrow \mathbb{R}$ given by the density $d(t, \mathbf{X})$ defined by

$d(t, \mathbf{X})=1-\frac{\int_{B_{\delta}(\mathbf{X})} \mu\left(t, \mathbf{X}, \mathbf{X}^{\prime}\right) d \mathbf{X}^{\prime}}{\int_{B_{\delta}(\mathbf{X})} d \mathbf{X}^{\prime}}$ 


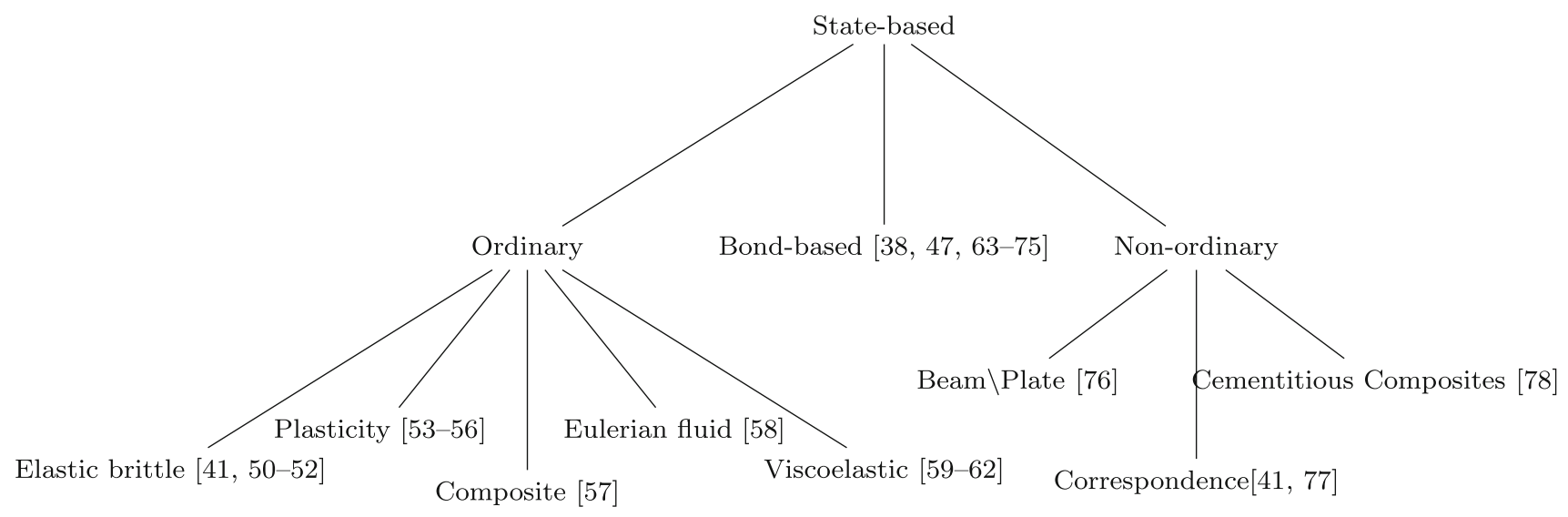

Fig. 2 The classification of the different peridynamic material models visualized as a tree. The material models are classified in two major classes: bond-based and state-based material models, respectively. State-based material models are distinguished as ordinary and non-ordinary models. The following ordinary state-based models are available: elastic-brittle, plasticity, composite, Eulerian fluid, and viscoleastic. For non-ordinary state-based models the Beam\Plate, the correspondence model, and a model for cementitious composites are available. Adapted from $[3,4]$ and extended for this work

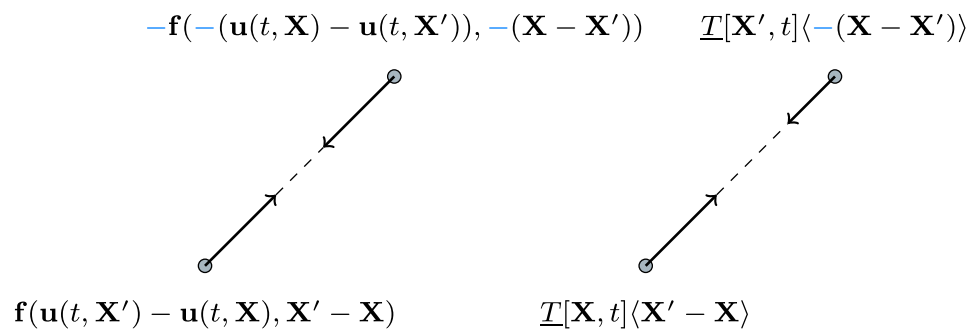

(a) Bond-based model (b) Ordinary state-based model

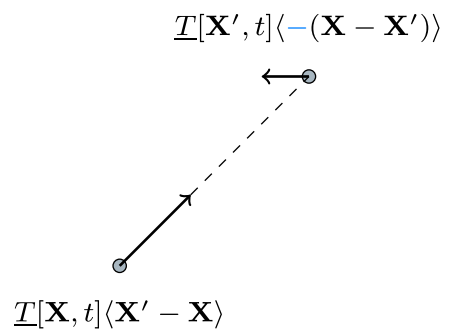

(c) Non-ordinary state-based model

Fig. 3 Schematics of the a bond-based model, $\mathbf{b}$ the ordinary state-based model, and $\mathbf{c}$ the non-ordinary state-based model. Note that the blue text in the Equations highlights the different assumptions for each model. This figure was adapted from [41]

where the scalar function $\mu:[0, T] \times \mathbb{R}^{n} \times \mathbb{R}^{n} \rightarrow \mathbb{R}$ indicates if the bond between $\mathbf{X}$ and $\mathbf{X}^{\prime}$ at time $t$ is broken $(\mu=0)$ or active $(\mu=1)$. Locations where $d=0$ indicate the crack set. The set where $d=0$ is highly localized and indicates the evolving crack path. This damage localization has been demonstrated mathematically by Lipton [46,47] for peridynamic fracture evolution corresponding to the small deformation bond based models. The method uses Gronwall's inequality together with methods from the non-local image approximation of the Mumford Shah model [48] introduced by Gobbino [49]. For more theory of peridynamic states, we refer to [41, Section 2]. For additional references, we refer to $[9,10]$. For reviews about $\mathrm{PD}$ and the comparison with experimental data, we refer to $[3,4]$.

\subsubsection{Material models for PD}

Figure 2 shows the tree of different peridynamic material models. The root are state-based material models, with the two subclasses of ordinary state-based and non-ordinary state-based material models. Note that bond-based mod- els [38,47,50-62] are a special case of state-based models. However, the bond-based PD was presented in 2000 and state-based PD in 2007. For ordinary state-based PD, the following material models are available: Elastic brittle [41,6365], Plasticity [66-69], Composite [70], Eulerian fluid [71], position-aware linear solid (PALS) [72], and Viscoelastic [73-76]. For non-ordinary state-based PD the correspondence model [41,77], the beam/plate model [78], and a model for cementitious composites [79] are available. For more details, we refer to [3].

Figure 3 shows the schematics of the three different models. All of these three models conserve linear momentum. However, only the bond-based model and the ordinarystate-based model conserve angular momentum. For more mathematical details, we refer to [41, Definiton 8.4].

\subsubsection{Discretization methods for PD}

Continuous and discontinuous finite element methods [8082], Gauss quadrature [83], and spatial discretization [38,84, $85]$ were utilized to discretize the peridynamic equation of 


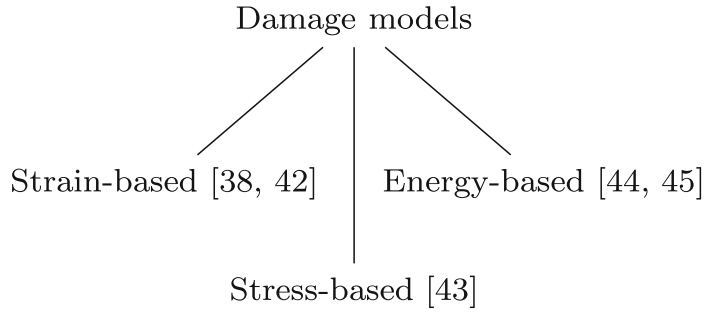

Fig. 4 The classification of different peridynamic damage models visualized as a tree. The majority of the damage models are based on strain, and only a few are based on energy or stress

motion. However, the most common discretization approach is the EMU nodal discretization, a colocation approach, introduced by Silling in [38]. In this approach, the domain $D$ is discretized with the discrete points $\mathbf{X}=\left\{\mathbf{x}_{i} \in \mathbb{R}^{n} \mid i=\right.$ $1, \ldots, N\}$ with the surrounding volumes $V=\left\{v_{i} \in \mathbb{R} \mid i=\right.$ $1, \ldots, N\}$. The following assumptions are made: all the volumes are non-overlapping $v_{i} \cap v_{j} \neq \emptyset$ for $i \neq j$ and the sum of all surrounding volumes is equal to the volume of the reference configuration $\sum v_{i} \approx V_{D}$. The discrete interaction zone is defined as $B_{\delta}\left(\mathbf{x}_{i}\right):=\left\{j \mid\left\|\mathbf{x}_{j}-\mathbf{x}_{i}\right\| \leq \delta\right\}$. The discrete bond-based equation of motion reads as

$$
\begin{aligned}
& \varrho\left(\mathbf{x}_{i}\right) \ddot{\mathbf{u}}\left(t, \mathbf{x}_{i}\right) \\
& \quad=\sum_{j \in B_{\delta}\left(\mathbf{x}_{i}\right)} \mathbf{f}\left(\mathbf{u}\left(t, \mathbf{x}_{j}\right)-\mathbf{u}\left(t, \mathbf{x}_{i}\right), \mathbf{x}_{j}-\mathbf{x}_{i}\right) d V_{j}+\mathbf{b}\left(t, \mathbf{x}_{i}\right)
\end{aligned}
$$

and the state-based equation of motion reads as

$$
\begin{aligned}
& \varrho\left(\mathbf{x}_{i}\right) \ddot{\mathbf{u}}\left(t, \mathbf{x}_{i}\right) \\
& \quad=\sum_{j \in B_{\delta}\left(\mathbf{x}_{i}\right)}\left(\underline{T}\left[\mathbf{x}_{i}, t\right]\left\langle\mathbf{x}_{j}-\mathbf{x}_{i}\right\rangle-\underline{T}\left[\mathbf{x}_{j}, t\right]\left\langle\mathbf{x}_{i}-\mathbf{x}_{j}\right\rangle\right) d V_{j} \\
& \quad+\mathbf{b}\left(t, \mathbf{x}_{i}\right) .
\end{aligned}
$$

The following implementations are available: Peridigm [86,87] and PDLammps [84] based on the Message Passing Interface (MPI), NonLocal models/PeriHPX $[88,89]$ based on the $\mathrm{C}++$ standard library for parallelism and concurrency (HPX) [90,91], PeriPy [92] based on the Python programming langugae, and GPU-based codes [93-95]. Four open source implementations of peridynamic PDLammps ${ }^{4}$, Peridigm $^{5}$, $\mathrm{PeriPy}^{6}$, and PeriHPX ${ }^{7}$ are available. One commercial code is available. LS-DYNA provides a bond-based peridynamics implementation discretized with the discontinuous Galerkin FEM [96]. Not to forget one of the first

\footnotetext{
${ }^{4}$ https://lammps.sandia.gov/doc/pair_peri.html.

${ }^{5}$ https://github.com/peridigm/peridigm.

${ }^{6}$ https://pypi.org/project/peripy/.

${ }^{7}$ https://perihpx.github.io/.
}

peridynamic implementations, EMU by Stewart Silling using FORTRAN 90 [97].

\subsubsection{Numerical analysis for PD fracture models}

In this section, we summarize the issues that arise in the numerical analysis of PD fracture models and list the numerical results. The following basic questions for PD fracture models are:

1. Are peridynamic fracture models well-posed, such that unique solutions exist?

2. What is the relation between non-local continuum peridynamic fracture models and their discretizations used in the numerical implementation?

3. How do PD solutions to fracture mechanics problems relate to local fracture models with sharp cracks? Particularly, how does PD relate to the more classical Linear Elastic Fracture Mechanics of continuum mechanics?

These are natural questions to ask, and analogous questions have been investigated and answered for several nonlocal and PD models in the absence of fracture, for this case there is now a vast literature; see [98-116]. This work provides the foundation for the numerical analysis of the PD fracture problem and this review concentrates exclusively on the PD fracture problem.

For the case of fracture, the analysis for PD fracture models is still in the initial stages, but meaningful progress has been made, and one can begin to address the three fundamental questions raised in the first paragraph:

First, the answer to question (1) is addressed. The existence and uniqueness of solutions for peridynamic fracture models have been studied for different classes of constitutive laws. For a simple peridynamic model with nonlocal forces that soften beyond a critical strain, the existence and uniqueness of the solution over finite time intervals is demonstrated for bond-based and state-based peridynamics in Lipton [46,47] and Jha \& Lipton [117]. Energy balance is shown to hold for all times of the evolution. This is a simple constitutive model designed for monotonically increasing loads.

Existence and uniqueness is established for more complex material models with the force degradation law determined by both the time and strain rate for strains above a critical value in Emmrich and Puhst [118]. Therein, both existence and uniqueness are established for bond-based peridynamic fracture. The authors Du et al. [119] consider a continuous version of the Prototypical Microelastic Bond (PMB) model introduced by Silling [37]. In this work both existence and uniqueness are shown and the total energy of the system is decreasing with time, see [119]. Existence and uniqueness is established for a state-based model with material degradation law, again determined by both the time and strain rate 
for strains above a critical load in Lipton et al. [120]. There, the rate form of energy balance is established among energy put into the system the kinetic energy, elastic energy, and energy dissipated due to the damage. The energy dissipation rate due to damage is seen to be positive. This model is suitable for cyclic loads, see [120]. The theme common to all peridynamic models is that both the existence and uniqueness of solutions follow from the Lipschitz continuity of the peridynamic force and the theory of vector-valued ODE on Banach spaces.

Second, the answer to question (2) is addressed. The convergence of finite difference approximations to bond-based and state-based peridynamic field theories with forces that soften is established in Jha \& Lipton [121] and [122]. The finite element convergence for bond-based and state-based peridynamic field theories with forces that soften are established by those authors in [123] and [81]. A priori convergent rates are linked to the regularity of continuum PD fracture solutions. Existence and uniqueness of solutions in Hölder spaces, and Sobolev spaces $H^{n}, n=1,2$, are proved for both bond and state based force softening models in [81,122]. The convergence rates for both bond- and state-based models are found to be linear in the mesh size and time step. However the constants appearing in the convergence estimates grow exponentially as the horizon size tends to zero. Fortunately, dynamic fracture experiments last hundreds of microseconds for brittle materials and linear a priori convergence rates for horizons that are tens of times smaller than the sample size are in force for tens of microseconds. Numerical experiments exhibit much better convergence with respect to mesh size and time step thus driving the need for the development of a posteriori estimates for understanding convergence rates.

Third, the answer to question (3) is addressed. For certain PD models one can theoretically recover a local sharp fracturing evolution. A limiting local evolution is shown to exist for the force softening peridynamic model; see [46,47]. The limiting local evolution has jump discontinuities in the displacement confined to a set of finite surface areas (more precisely, two-dimensional Hausdorff measure) for almost every time; see $[46,47]$. The jump set corresponds to the fracture set in the zero horizon model and the total energy is bounded and given by the classic energy of linear elastic fracture mechanics $[46,47,124]$. It is shown there that the deformation in the limit model satisfies the local balance of linear momentum equation in quiescent zones away from the crack. Recent work explores the zero horizon limit for straight cracks growing continuously with the goal of capturing the explicit interaction between the growing crack and the surrounding elastic material. For this case, it has been found by Lipton et al. [125] that the local model obtained in the zero horizon limit is given by a deformation field, that is, the weak solution of the linear wave equation on the domain with the growing crack satisfies the zero traction condition of the sides of the crack. This is in agreement with Linear Elastic Fracture Mechanics (LEFM). Here, the weak solution of the wave equation outside a time-dependent domain defined by a crack was recently developed in DalMaso and Toader [126]. The convergence of PD to the wave equation in time-dependent domains [125] gives theoretical support backing the recent development of new "asymptotically compatible" methods for fracture modeling given in Trask et al. [127]. Lastly, starting with the PD equation multiplying by the velocity and integrating by parts gives the time rate of change of internal energy surrounding the crack front. Jha \& Lipton [128] use applied math arguments to show that on passing to the zero horizon limit, the kinetic relation for crack tip growth given by LEFM is recovered. Here the classic square root singularity in the elastic field at the crack tip is obtained.

We conclude this section noting that the numerical analysis of PD in the absence of fracture provides compelling heuristics for understanding PD fracture models. Figure 5 illustrates the interplay between horizon length scale and discretization length scale for PD models when local models can be recovered by passing to the small horizon limit in non-local models, see Du et al. [111,112]. When a numerical scheme can be designed so that the diagonal arrow captures the same limit as obtained by proceeding along the sides of the square problem, a numerical scheme is said to be asymptotically compatible. This is the motivation behind the numerical approach of Trask et al. [127] to capture the coupling between intact material surrounding a growing crack. For example, if one considers elastic problems in the absence of fracture, then for the diagonal transition where the horizon $\delta$ and the nodal spacing $h$ go to zero, it is known that:

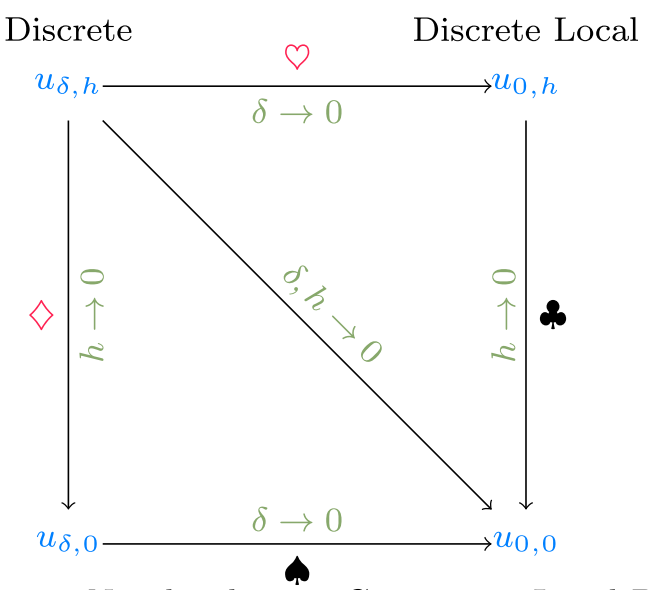

Continuum Non-local

Continuum Local PDE

Fig. 5 The consistency of non-local models and the limits of the horizon $\delta$ and the nodal spacing $h$ presented in Du et al. [111,112]. Adapted from [129] 
using piece-wise constant finite elements, the correct local solution is obtained, if the nodal spacing decays faster than the horizon to zero [111]. This is seen for the EMU nodal discretization [97] which converges to the limit $u_{0,0}$ along the diagonal if the nodal spacing decays faster than the horizon in Tian and Du [114].

\subsubsection{On the connection between meshfree peridynamics and other meshfree methods}

In the absence of fracture, the relation of PD to molecular dynamics (MD) has been shown by Seleson et al. [130]. Along another direction, the relation of PD to smooth-particle hydrodynamics (SPH) is established in Ganzenmüller et al. [110]. With these studies in mind, it is clear that up-scaling MD fracture models to PD and establishing the relation between SPH and PD for fracture would be desirable.

Bessa et al. [131] showed that approximated derivatives of state-based PD are completely equivalent to the approximation received by the reproducing kernel particle method (RKPM) with synchronized derivatives and with a quadratic polynomial basis. Bode et al. [132] introduced the peridynamic Petrov-Galerkin (PPG) method, which is developed based on the PD equation of motion starting from the statebased formulation in [10]. Hillman et al. [133] showed the relation between reproducing kernel (RK) approximation with implicit gradient and peridynamic. Madenci et al. [134] introduced the peridynamic differential operator (PDDO). Shojaei et al. [135] introduced a generalized finite difference method (GDFM) based on PDDO. Table 1 lists all of these connections. Fore more details, we refer to [8].

\subsubsection{Visualization of PD results}

Since most peridynamic simulations are done using a meshless method, information, e.g. stress and strain, are only available on the discrete nodes. Thus, every graphics soft-

Table 1 Connection between meshless discrete PD and other meshfree methods. Adapted and extended from [8]

\begin{tabular}{lll}
\hline Method & Equivalence & Reference \\
\hline $\begin{array}{l}\text { Smooth-particle } \\
\text { hydrodynamics }\end{array}$ & Deformation gradient & {$[110]$} \\
$\begin{array}{c}\text { Reproducing kernel } \\
\text { particle method }\end{array}$ & Deformation gradient & {$[131]$} \\
$\begin{array}{c}\text { Petrov-Galerkin method } \\
\begin{array}{c}\text { Reproducing kernel } \\
\text { with implicit gradient }\end{array}\end{array}$ & $\begin{array}{c}\text { Correspondence for } \\
\text { force state }\end{array}$ & {$[132]$} \\
$\begin{array}{c}\text { Generalized finite } \\
\text { difference method }\end{array}$ & $\begin{array}{c}\text { Peridynamic differential } \\
\text { Operator [134] }\end{array}$ & {$[135]$} \\
\hline
\end{tabular}

ware, e.g. Paraview [136] or VisIt [137], supporting particles can be used to visualize meshless simulation results. However, to understand the simulation and compare against experimental data, this information is needed on a larger scale. First, peridynamic theory was used for physicallybased modeling and rendering. Here, the animation of brittle fracture [138], the animation of fractures in elastoplastic solids [139], and the animation of hyper elastic materials [140] were studied. Second, the visualization of fragmentation [141,142] and visualization of fracture progression [143] were investigated. For more details, we refer the readers to [4].

\subsection{Phase-field models governing equations}

Variational models to fracture and damage models were introduced by Francfort \& Marigo [144] and Aranson et al. [145]. A numerical approximation of variational fracture models was first introduced by Bourdin et al. [146]. We also refer to [12] and the recent review paper [15].

The principal idea of the variational (phase-field) approach to fracture (here explained for quasi-static fracture in brittle materials) is based on energy minimization in which potential and fracture energies interact. To this end, let $\Omega \subset \mathbb{R}^{n}$ be the intact domain and $\Gamma \subset \mathbb{R}^{n-1}$ the fracture set. Let $\mathbf{u}: \Omega \rightarrow \mathbb{R}^{n}$ be a displacement field and the total energy be given by

$$
E(\mathbf{u}, \Gamma)=\underbrace{\int_{\Omega} \psi_{0}(\varepsilon(\mathbf{u})) d V-F(\mathbf{u})}_{=: P(\mathbf{u})}+\underbrace{\int_{\Omega} G_{c} d A}_{=\psi_{c}(\Gamma)},
$$

with the potential energy

$$
P(\mathbf{u}):=\int_{\Omega} \psi_{0}(\varepsilon(\mathbf{u})) d V-F(\mathbf{u})
$$

composed by the bulk energy (first term) with $\psi_{0}(\varepsilon(\mathbf{u})):=$ $\mathrm{C} \varepsilon(\mathbf{u}) \cdot \varepsilon(\mathbf{u})$ being the energy storage function with the stiffness tensor $\mathrm{C} \in \mathbb{R}^{n \times n \times n \times n}$, and the linearized strain tensor $\varepsilon(\mathbf{u})=\frac{1}{2}\left(\nabla \mathbf{u}+\nabla \mathbf{u}^{T}\right)$. The external potential of volume and surface forces is given by

$$
F(\mathbf{u})=\int_{\Omega} \mathbf{b}^{*} \mathbf{u} d V+\int_{\partial \Omega_{t}} \mathbf{t}^{*} \mathbf{u} d A,
$$

where $\mathbf{b}^{*}$ is the distributed body force and $\mathbf{t}^{*}$ are traction forces. The crack surface energy is given by

$$
\psi_{c}(\Gamma)=\int_{\Gamma} G_{c} d A
$$


where $G_{c}>0$ is the critical energy release rate.

A corresponding configuration is sketched in Fig. 6a. Therein, the domain $\Omega$ of the solid with a (sharp) crack set $\Gamma$ is considered. For the boundary $\partial \Omega$ of the domain $\Omega$ two kinds of boundary conditions along the normal vector $\mathbf{n}$ are considered such that $\partial \Omega_{u} \cap \partial \Omega_{t}=\varnothing$. On the boundary $\partial \Omega_{u}$ Dirichlet displacement conditions are applied, which are built, as usually done, into the governing function spaces. Tractions $\mathbf{t}^{*}$ are applied to the $\partial \Omega_{t}$ boundary. Discussions on boundary conditions can be found for instance in the early work [144,147,148].

From mathematical and numerical viewpoints, the sharp fracture representation (12) is challenging because the crack 'lives' on a lower-dimensional manifold $\Gamma$. On the one hand, this requires special function spaces (see e.g., [147,148]), and on the other hand, numerical approximations require specialized discretizations (for instance generalized/extended finite elements [22-24] among various other possible techniques).

To handle this challenge one can borrow techniques from image processing. The single-well Modica-Mortola functional is introduced by Ambrosio and Tortorelli [149,150] in image processing to approximate the the surface area term in the Mumford-Shah functional [48] and is given by

$$
\int_{\Gamma} d A \approx \int_{\Omega_{c}} \gamma(\varphi, \nabla \varphi) d V
$$

with

$\gamma(\varphi, \nabla \varphi)=\frac{1}{2}\left[\frac{1}{l_{0}}(1-\varphi)^{2}+l_{0}|\nabla \varphi|^{2}\right]$.

Bourdin et al. [146] proposed to use this energy in an appealing approach to regularize the sharp crack defined on $\Gamma$ by a domain integral defined on $\Omega_{c}$. In this context it is

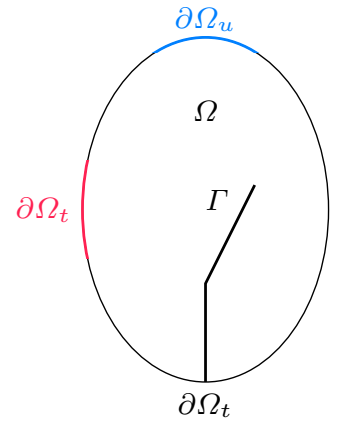

(a)

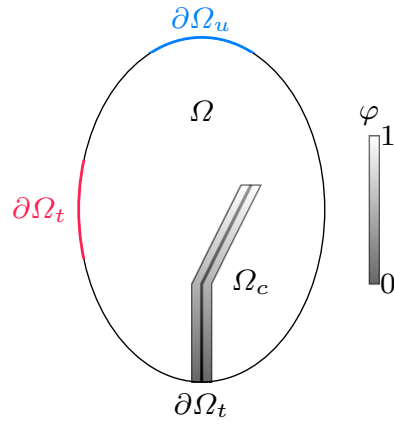

(b)
Fig. 6 The solid phase-field domain $\Omega$ with a a sharp crack interface $\Gamma$ and $\mathbf{b}$ the approximated crack using the phase-field crack function $\varphi$ resulting in a regularized crack representation $\Omega_{c}$ given by

$\int_{\Gamma} G_{c} d A \approx \int_{\Omega_{c}} G_{c} \gamma(\varphi, \nabla \varphi) d V$

with $\gamma(\varphi, \nabla \varphi)$ is now viewed physically as the crack surface density function as in [151]. Here $l_{0}>0$ is the so-called length scale (i.e., regularization) parameter and $l_{0}$ characterizes the width of the regularized domain $\Omega_{c}$. Note that there are other formulations for the crack surface density function available [152,153].

The name 'phase-field' was first coined in the year 2008 in the $\mathrm{PAMM}^{8}$ proceedings from Kuhn and Müller [154] and in their 2010 follow-up journal paper [155]. In the same year, Miehe et al. [151] used this terminology, which includes the above regularization, but additionally, they justified from a mechanical perspective a thermodynamically consistent framework for the potential and fracture energies. We notice that simultaneous pioneering work in variational gradient damage evolutions was performed by Pham and Marigo $[156,157]$ for which we also refer the reader to Section 2.2.3.

In other words, within phase-field models the crack is regularized by $\Omega_{c}$ around the sharp crack $\Gamma$ using the so-called phase-field crack function $\varphi: \Omega_{c} \cup \Omega \rightarrow[0,1]$, see Fig. 6b. The notation is that $\varphi=0$ indicates damage and $\varphi=1$ means intact material (some authors define it the other way around). Between $\varphi=0$ and $\varphi=1$, the function varies smoothly with values $0<\varphi<1$, which is the so-called transition zone.

Figure $6 \mathrm{~b}$ sketches the situation in which the smoothed region $\Omega_{c}$ is shown.

By using the phase-field variable, the bulk strain energy is extended to the entire domain (intact domain plus fractured domain) and we obtain

$\psi_{s}(\mathbf{u}, \varphi)=\int_{\Omega \cup \Omega_{c}} g(\varphi) \psi_{0}(\varepsilon(\mathbf{u})) d V$,

where $\psi_{0}$ is the so-called non-degraded bulk strain energy and $g(\varphi)$ the so-called degradation function. Usually, $g(\varphi)=$ $\varphi^{2}+\kappa$ or $g(\varphi)=(1-\kappa) \varphi^{2}+\kappa$ with a small $\kappa>0$, which is necessary to ensure regular system matrices within the discretization for quasi-static fracture. Clearly, for $\varphi=0$ (fracture), we have $g(\varphi)=\kappa$. In the non-fractured region, we have $\varphi=1$ and $g(\varphi)=1$. For dynamic fracture (see a discussion in [158]) $\kappa=0$ is possible, which is obvious because the mass matrix term arising from the discretization of the acceleration term ensures well-posedness of the discrete system. A family of degradation functions and their

\footnotetext{
${ }^{8}$ PAMM $=$ Proceedings in Applied Mathematics and Mechanics
} 
numerical justification was investigated in [159] and a multidimensional stability analysis with a general degradation function was considered in [160]. The mathematical relation between $l_{0}$ and $\kappa$ is linked to $\Gamma$ convergence in which $l_{0} \rightarrow 0$ and $\kappa \rightarrow 0$ with the asymptotic behavior $\kappa=o\left(l_{0}\right)$; see e.g., [161] and again the approximation results in [149,150].

Summarizing the previous ingredients, the total energy using the regularized crack representation in the entire domain $\Omega \cup \Omega_{c}$ is given by

$$
\begin{aligned}
E(\mathbf{u}, \varphi)= & \int_{\Omega \cup \Omega_{c}} g(\varphi) \psi_{0}(\varepsilon(\mathbf{u})) d V-F(\mathbf{u}) \\
& +\int_{\Omega \cup \Omega_{c}} G_{c} \frac{1}{2}\left[\frac{1}{l_{0}}(1-\varphi)^{2}+l_{0}|\nabla \varphi|^{2}\right] d V .
\end{aligned}
$$

Several general types of $\psi_{0}$ functions have been proposed, and it was shown that a suitable choice could avoid nonphysical growth of cracks under compressive loading [162]. For more details, we refer to $[16,163]$.

Moreover, in most studies, as model assumption from a physics perspective, the crack cannot heal, and therefore the above energy functional is subject to a crack irreversibility (an entropy condition), which is mathematically expressed as an inequality constraint in time or quasi-static loading:

$\partial_{t} \varphi \leq 0$

Due to this constraint, we deal with a quasi-static incremental (or time-dependent) nonlinear, coupled variational inequality system. The solution is obtained by minimizing $E(\mathbf{u}, \varphi)$ with respect to $\mathbf{u}$ and $\varphi$ by considering $\partial_{t} \varphi \leq 0$. Therein, the bulk and crack energies interact according to the laws outlined in [144] and [151].

\subsubsection{Properties of $\phi$ and crack interface reconstruction}

It can be rigorously proven with cut-off arguments that $\varphi \in[0,1]$; see for instance [164], which follows from the definition of the Ambrosio-Tortorelli functional and the regularization of the total energy. When further terms (physics) are added, the property $\varphi \in[0,1]$ may get lost, and one must argue carefully. For instance, in pressurized fractures, the pressure can have positive and negative values and, therefore, further cut-off arguments are necessary in order to establish the bounds for $\varphi$ [165]. As the second topic in this short paragraph, we want to mention the principal idea when the crack interface must be known explicitly. Due to the regularization using $\varphi$, there is some liberty as to when the exact crack interface must be known. In these cases, the phase-field function is interpreted as a level-set function ([166]) and the crack interface is, for instance, chosen as $\varphi_{c}:=\varphi=c_{I}$ with for example $c_{I}=0.2[17,167,168]$.

\subsubsection{Brief review of some theoretical findings}

We briefly list some important well-posedness results. In [169] first existence results for quasi-brittle fracture of the original model by Francfort \& Marigo [144] were shown for the antiplane setting for scalar-valued displacements. In [147] the existence and convergence of quasi-static evolutions for the vector-valued case were established. Shortly after, the existence of quasistatic crack growth in nonlinear elasticity was proven [148]. For these settings, in general, uniqueness cannot be established; see also below in Sect. 2.2.3 for discussions and references in the related gradient damage theory. In fact, it is well-known that uniqueness is a general issue in solid mechanics; see e.g. [170].

Regarding uniqueness in phase-field fracture, we deal with two variables, namely $\varphi$ and $\mathbf{u}$, obtained from solving a nonlinear coupled system, and the governing functional to be minimized is not convex, yielding several local minima. Only recently in [171], the issue of non-uniqueness was investigated in detail by using a stochastic approach by computing all solutions with their respective probability in which they may occur.

The existence of solutions for dynamic fracture using Ambrosio-Tortorelli [149,150] approximations was established in [172]. Since crack initiation is an important topic within phase-field based crack models, we mention theoretical work by Chambolle et al. [173], Goethem/Novotny [174], and recently Kumar et al. [175] and de Lorenzis/Maurini [176]. Some theoretical findings on the crack path were provided in [177]. For mode III dynamic fracture modeled using [172], one can follow a sequence of solutions as $l_{0} \rightarrow 0$, to obtain existence of a limiting displacement with bounded Linear Elastic Fracture Mechanics energy [47]. The latest review of the original model, in terms of the sharp crack approximation (without phase-field, but nonetheless the ground basis of regularized models such as phase-field) can be founded in recently published article by Francfort [18]. Furthermore, we refer to the SIAM News article [15].

\subsubsection{Fracture/damage models for PF}

In this section, we first note that phase-field fracture models have a close relationship to damage models. Indeed, due to the regularization in phase-field models yielding a transition zone, and damage mechanics with gradients, similar approaches are obtained. For seminal work in the year 2010, we refer to Pham and Marigo [156,157] (based on earlier work of Mielke [178]). Therein, the authors propose and investigate local [156] and nonlocal [157] brittle damage laws from a mechanical viewpoint. The evolution is based on three fundamental physical principles: irreversibility of the damage (i.e., fracture) parameter, stability, and an energy balance. The actual relation of gradient damage models 
Table 2 Overview of various fracture and damage models available for phase-field modeling. Adapted and extended from [16]

\begin{tabular}{ll}
\hline Fracture/damage models & \\
\hline Brittle fracture & {$[12,146,151,154,155,186]$} \\
Ductile fracture & {$[199,225,248-252]$} \\
Cohesive fracture & {$[12,239,242-245,281]$} \\
Dynamic fracture & {$[172,221,222,235-237,241,282]$} \\
Fracture in incompressible & {$[283-285]$} \\
solids & \\
Finite deformation fracture & {$[217,227,229-232]$} \\
3D fracture & {$[151,202,221-228]$} \\
Plate/shell fracture & {$[214-220]$} \\
Multi-field fracture & {$[152,197-210,286]$} \\
Fatigue & {$[189-193]$} \\
Layered material fracture & {$[215,244,265-271]$} \\
Anisotropic surface energy & {$[259-264]$} \\
\hline
\end{tabular}

with brittle fracture is described in [179]. Further properties of such damage models (and consequently phase-field approaches) are investigated in [180]. Specifically, the stability and uniqueness (in terms of bifurcation) of the evolution of the gradient damage variational formulation is investigated for uniaxial tests, namely the homogeneous response of a bar under an increasing traction loading. The construction of bifurcations for a large class of gradient damage models (i.e., elastic-softening materials) was continued in [181]. Earlier work on stability and bifurcations using energetic variational principles is from Nguyen $[182,183]$. Solely bifurcations for a one-dimensional gradient damage model applied to a bar were considered in [184]. Here, the mathematical ill-posedness (aspects of uniqueness and dependencies on the data) is discussed and possible consequences of numerical approximations (in particular mesh sensitivity) is drawn (here, we also point to [171] for uniqueness studies for phase-field fracture). Another, more recent discussion about similarities and differences can be found in [185]. In what follows, we therefore have both fracture and damage models in mind. Table 2 lists the available phase-field fracture and damage models. The following models have been developed within the phase-field framework: brittle fracture [12,146,151,155,186-188], fatigue [189-196], multi-field fracture [152,197-212], frictional contact [213], plate/shell fracture [214-220] (where [220] solves some issues with the energy decomposition which were leading in [214,216] to unphysical results), three-dimensional fracture $[151,202$, 221-228], finite deformation/hyperelastic fracture [217,227, 229-234], dynamic fracture [172,221,222,235-241], cohesive fracture [12,242-247], ductile fracture [199,225,248258], anisotropic surface energy [259-264], layered material fracture [215,244,265-271], and polymer composites fracture [272].
Furthermore, we summarize various models for splitting the energy (i.e., strain / stress) into different parts for accounting the fracture growth behavior under tension and compression. To the best of our knowledge, we are aware of Amor et al. [273], Miehe et al. [151], Zhang et al. [274], Strobl/Seelig [275], Steinke/Kaliske [276], Bryant/Sun [277], Freddi/Royer-Carfagni [278], Bilgen/Homberger/Weinberg [279], and Fan et al. [280].

\subsubsection{Treating the crack irreversibility constraint}

For treating the irreversibility constraint $\partial_{t} \varphi \leq 0$, six fundamental procedures have been proposed:

1. Fixing crack nodes by Dirichlet values $[146,155]$;

2. Strain history function [186];

3. Penalization: simple and augmented Lagrangian [165, 287-289];

4. Primal-dual active set methods [290];

5. Complementarity system with Lagrange multipliers as unknowns [283];

6. Interior-point methods [291].

Comparisons of some of these approaches were performed in [288] and [17].

\subsubsection{Discretization, solvers, and software for PF}

Classical Lagrange Galerkin finite elements [146], exponential shape functions [292], isogeometric elements [222], discontinuous finite elements [293], or mixed formulations [283] were mostly utilized for the spatial discretization of the fracture/damage models as described in the previous section. Meshless methods for general phase-field were first introduced in [294] and for meshless phase-field fracture, see e.g., [216,295]. For discretized nonlinear systems, the following solvers are available: alternating minimisation algorithms [146,153,187,237,273,296-298], alternating minimisation algorithm with path-following strategies [299], staggered scheme [186], stabilized staggered schemes [300303], monolithic solvers [17,151,290,304-308], and monolithic solvers with path-following strategies [309,310].

For solving linear equation systems, most often blackbox (direct) solvers have been adopted. Only recently [297] proposed conjugate gradient (CG) solutions with multigrid preconditioning for the decoupled phase-field displacement system. For monolithic solvers, a generalized minimal residual (GMRES) method with parallel algebraic multigrid preconditioning was proposed in [311]. A matrix-free geometric multigrid preconditioner was developed in [307], and its parallelized variant in [312]. Furthermore, we mention the development of a FFT (fast Fourier transform) solver for higher-order phase-field fracture problems [313]. 
The following proprietary implementations using Matlab [314,315], COMSOL [316], [317,318] using FEAP ${ }^{9}$, and Abaqus [226,319-324] are available. Open source implementations are: [310] using Nutils [325], [243] using JIVE ${ }^{10}$, [326] using MOOSE [327], [328] (pfm-cracks ${ }^{11}$ and [329] using deal.II ${ }^{12}$, and [237,297] using FENICS [330] are available. In addition, a GPU-based implementation [331] and the MEF 90 Fortran implementation [12,144,296,332] are available.

\subsubsection{Numerical analysis for PF}

A posteriori error estimation For numerical analysis with respect to a posteriori error estimation, a short summary is presented here. First, work on residual-based error estimators goes back to [187,333]. Extracting error indicators for local mesh refinement based on an a posteriori error estimator for the phase-field variational inequality are presented in [334,335] The development of goal-oriented mesh adaptivity was undertaken in $[17,336]$. The Ambrosio-Tortorelli functional is used to $\Gamma$ approximate each time evolution step in [337]. An additional penalty constraint is enforced for the irreversibility of the fracture as well as the applied displacement field. An a posteriori error estimator driving the anisotropic adaptive procedure is utilized for mesh adaptivity. According to the authors, the main properties of automatically generated meshes are to be very fine and strongly anisotropic in a small neighborhood region of the crack, but only far away from the crack tip, while they show a highly isotropic behavior in a neighborhood of the crack tip instead. The Ambrosio-Tortorelli functional is applied in [187] to two adaptive finite element algorithms for the computation of its (local) minimizers. Two theoretical results demonstrate convergence of the developed algorithms to the local minimizers of the Ambrosio-Tortorelli functional. However, the Ambrosio-Tortorelli functional is for quasi-static simulations and might not apply to dynamic fracture situations. The phase-field parameter itself is used in [158] to refine the mesh. The gradients of the phase-field are high in the near crack region and close to one away from the crack. A threshold is introduced to run the dynamic phase-field simulation for a few time steps, then all elements are refined above the introduced threshold, and the simulation is resumed with the newly refined mesh. This procedure is repeated until the convergence criterion is met.

It is expected that improved error estimates can further advance both PD and PF modeling approaches to pave the

\footnotetext{
$\overline{9}$ http://projects.ce.berkeley.edu/feap/.

${ }^{10} \mathrm{http} / / / \mathrm{www} . j \mathrm{jem}$-jive.com.

$11 \mathrm{https}: / /$ github.com/tjhei/cracks.

12 https://www.dealii.org/.
}

path for routine use as predictive simulations for a certain class of fracture problems.

Goal functional evaluations and computational analysis for $l_{0}-h$ relationship In $[17,336,338]$ a slit domain (a square plate with an initial crack) with displacement discontinuity at the crack and the manufactured displacement field $[339,340]$ are utilized to study the $l_{0}-h$ relationship. Note that the crack in this study is represented by the phase-field damage function $\varphi$. Motivated by [161,341], various simulations for $l_{0}=c h^{l}$ with $l \in(0,1]$ and $h$ as the mesh size are conducted. Three cases of mesh refinement are studied: 1 ) $c=2.0$ and $l=1.0,2) c=0.5$ and $l=0.5$, and 3) $c=0.5$ and $l=0.25$. First, the influence of $l_{0}$ on the goal function evaluation is considered. Therefore, the goal function $J\left(u_{\mathrm{FM}}\right):=u_{\mathrm{FM}}(0.75,-0.75)$ for a displacement point value is utilized, which results in the total error $J\left(u_{\mathrm{FM}}\right)-J\left(u_{h}\right)$. The maximal convergence order of $r=\mathcal{O}\left(l_{0}\right)$ was obtained in case 2) where $r=l_{0}=0.5$. The observed order is $r=l_{0}=0.25$ for case 3 ) and $r \approx 0.9<l_{0}$ for case 1$)$. These results lead to the assumption that $\left|J\left(u_{\mathrm{FM}}\right)-J\left(u_{h}\right)\right|=\mathcal{O}\left(l_{0}\right)$ as presented in [202,290,300,311]. In addition, two phasefield fracture configurations were proposed as prototype models for comparison in the recent benchmark collection [342].

Adaptivity Regarding adaptivity, we distinguish between spatial and temporal mesh refinement and adaptive solution schemes. Spatial mesh refinement goes back to anisotropies introduced by the mesh [343], residual-based adaptive finite elements [187,333], anisotropic adaptive mesh refinement [337], and pre-refined meshes where the crack path is known a priori [222]. Other computational convergence analyses were undertaken in [344]. For unknown crack paths, a predictor-corrector approach was developed and applied in [202,290,345], goal-oriented error-control [336,338], and mesh refinement in multiscale phase-field methods [346]. A few rigorous studies on temporal error control exist [331,347]. Apart from classical mesh refinement, an adaptive predictor-corrector non-intrusive global-local (multiscale) approach [315] (see also [348]) was developed based on the approach presented in [349], and was to porous media [350], and extended to multilevel concepts [351].

Solver analyses Using alternating minimization for solving the coupled displacement-phase-field problem, the convergence of the scheme was established in [296] and [187]. A convergence proof for a truncated nonsmooth Newton multigrid method was very recently undertaken in [352]. For further fully-coupled (i.e., monolithic) techniques, no rigorous convergence are available, but significant numerical evidence of the performance of nonlinear solvers $[17,151$, 290,291,304-310,353].

In the following, we discuss some papers in more detail. Zhang et al. [354] used a length scale material parameter to evaluate the accuracy of phase-field modeling of brittle 
fractures with available experimental data. They observed significant discrepancies between numerical predictions and the experimentally observed load-displacement curves after the critical force, despite a reasonably accurate prediction of crack paths. Zhuang et al. [355] implemented the phase-field method in a staggered scheme to sequentially solve for the displacement, phase-field, and fluid pressure. Asymmetric deflection along material interfaces and penetration of hydraulic fractures in naturally-layered porous media were reported for different layer arrangements based upon their respective stiffness as measured by $E$ and $G_{c}$. Farrell and Maurini [297] reformulated the alternate minimization algorithm for the variational fracture approach to simulate nucleation and propagation of complex fracture patterns as a nonlinear Gauss-Seidel iteration along with over-relaxation to accelerate its convergence. They showed further reduction in solution time by utilizing the accelerated alternate minimization with Newton's method. Brun et al. [300] showed an iterative staggered scheme, a two-field variational inequality system with independent phase-field variable and displacement variables. For the convergence using a fix-point argument and a natural condition, the elastic mechanical energy remains bounded and with a sufficiently thick diffusive zone around the crack surface, monotonic convergence is achieved. Noll et al. [356] presented results for ductile fracture with linear isotropic hardening and discussed the computational costs for 3D simulations while analyzing added computations due to mesh refinement. Chukwudozie et al. [357] presented a unified fracture-porous medium hydraulic fracturing model that handled interactions among multiple cracks, as well as the evolution of complex crack paths in 3D simulations using energy minimization without any additional branching criterion, but the location of crack tip and its velocity remains a challenge in complex configurations. Further, detailed linear solver analyses for quasi-monolithic phase-field fracture using a GMRES solver with matrix-free geometric multigrid preconditioning were conducted in [307]. Scalability tests of parallel performance were performed in [311] and [312].

It is evident that further improvements in robust solvers will be the key for both PD and PF approaches to be adopted as the engineering tools of choice to predict fracture phenomena.

\section{Macroscale view of crack propagation physics using thermodynamics constraints and constitutive relationships}

According to Haslach [358], a maximum dissipation nonequilibrium evolution model can describe the unsteady crack propagation rate for both brittle fracture and for viscoplastic behavior at the crack tip. Ulmer et al. [359] presented a thermodynamically consistent framework for phase-field models of crack propagation in ductile elastic-plastic solids under dynamic loading with an incremental variational principle and validated it against the classical Kalthoff-Winkler experimental results. Mauthe and Miehe [360] used two constitutive functions - free energy and dissipation potential to incorporate fluid flow in cracks during hydraulic fracturing and coupled it to a phase-field approach to fracture within a variational framework. Miehe et al. [361] proposed a gradient damage formulation with two independent length scales to regularize the plastic response and crack discontinuities to ensure that the damage zones of ductile fractures remain inside plastic zones. Roy et al. [362] presented a rephrased phase-field theory of continuum damage in a peridynamics setup and showed promising results of mode II delamination. Farrahi et al. [363] demonstrated that under mode I crack growth and proper calibration of parameters, PFM always agreed with Griffith's theory. Alessi et al. [364] demonstrated that macroscopic responses assimilable to brittle fractures, cohesive fractures, and a sort of cohesive fracture, including depinning energy contributions by tuning a few key constitutive parameters such as relative yield stresses and softening behaviors of the plasticity and damage criteria. It is duly noted that both PD and PF show promise to visualize and predict complex fracture phenomena without resorting to ad-hoc modeling assumptions.

\section{Validation against experimental data}

The validation against experimental data is a cornerstone to access the predictive accuracy of any engineering fracture mechanics model. In this section, the experiments used as benchmarks for peridynamic models are compared against the ones used as benchmarks for phase-field models. We limited the focus to the Sandia Fracture Challenge and publications where both models were compared to the same experimental data. For a detailed review about the comparison with experimental data, we refer to [4] for peridynamic models and for phase-field models to [16, Section 2.12]. It is remarked that the experimental data from Jeffery and Bunger [365] may be used in the validation of different numerical simulators for hydraulic fracture propagation.

\subsection{Reasons for Sandia Fracture Challenge and outline}

Two reasons make the Sandia Fracture Challenge (SFC) an excellent example:

1. experimental data is usually not disclosed in the literature [366] whereas all data and experimental designs are made available in the Sandia challenge; 
2. the Sandia Fracture Challenge has two unique features that together establish the validity of a free fracture model. First, the calibration step requires the free fracture model to be calibrated through a simple prototypical problem, e.g. a tensile test. The subsequent validation step requires the calibrated model to simulate a distinct and more complicated fracture problem. It is the model's ability to match experimental results for the complex problem that establishes its validity. This prohibits parameter fitting to match a particular experiment.

The outline for the following subsections is as follows: we first provide the methodology for error measurements used to compare the different models. Afterward, the Sandia Fracture Challenge is described in detail, with a focus on the first and third test sets. These descriptions are followed by comparisons of both models, including details on the respective discretizations, numerical cost, and error measurements.

\subsection{Error measurements for comparisons}

For all compared values, the following error measurements were calculated: For scalar values, the relative error $\epsilon_{\text {rel }}=$ $\left(x_{\text {sim }}-x_{\text {exp }}\right) / x_{\text {exp }}$ and for a time series the coefficient of determination $R^{2}$ [367] is computed, when applicable. The coefficient of determination $R^{2}$ is defined as

$R^{2}=1-\frac{S S E}{S S T}$

for two series of $n$ values $y_{1}, \ldots, y_{n}$, the so-called series of observables and $\hat{y}_{1}, \ldots, \hat{y}_{n}$, the so-called series of predictions. Where the total sum of squares $S S T$ reads as

$S S T=\sum_{1}^{n}\left(y_{i}-\bar{y}\right)^{2} \quad$ with $\quad \bar{y}=\frac{1}{n} \sum_{1}^{n} y_{i}$

and the sum of square residuals (or errors) reads as

$$
S S E=\sum_{1}^{n}\left(y_{i}-\hat{y}_{i}\right)^{2} \text {. }
$$

Thus, $R^{2}$ is a statistical measure in the range of zero to one to indicate how good the series of predictions $\hat{y}_{i}$ approximates the series of observables $y_{i}$. Specifically, $R^{2}=1$ implies that $S S E=0$ and therefore, the series of observables fits the series of predictions perfectly. If $R^{2}=0$ and therefore $S S E=S S T$ then the mean of observables series is as good as any predicted series. For the time series, the WebPlotDigitizer ${ }^{13}$ was used to extract the $x$ and $y$ coordinates of the respective plot from the Sandia report.

$\overline{13}$ https://automeris.io/WebPlotDigitizer/.
The scipy.stats. Iinregres ${ }^{14}$ functionality of the python SciPy package [368] was utilized to compute the $R^{2}$ correlation.

\subsection{Sandia Fracture Challenge}

The Sandia Fracture Challenge is considered as one of the potential set of benchmarks to showcase the predictive accuracy of the two models for various complex experimental data. There are many other experimental data sets available which could serve as experimental benchmarks as well. However, the SFC addresses some important aspects of calibration vs. validation against experimental results. One essential part of this challenge is to calibrate the free fracture model on simple prototypical problems such as a tensile test, and use this calibration to simulate the crack and fracture phenomena. With this additional step, the model parameters can not be calibrated or fitted to the complex scenario but instead have been calibrated using the simple scenario. This additional step of calibration makes the SFC an excellent problem to benchmark phase-field models and peridynamic models to assess their performance on different kinds of crack and fracture phenomena. For the first and third fracture challenge, we could find the contributions of peridynamic models, see Sect. 4.3.1 and Sect. 4.3.2, respectively. No studies using phase-field models were found for all three fracture challenges. The summary of model accuracy is shown in Table 5.

\subsubsection{First Sandia Fracture Challenge}

In the first Sandia Fracture Challenge [369] blind round robin predictions of ductile tearing for an alloy $(15-5 \mathrm{PH})$ were studied. The stress-strain curve of a tensile bar was provided to calibrate the model. Experiments on CT specimens were conducted and the extracted quantities of interest are shown in Fig. 7. The geometry has a blunt notch $A$ and three holes $B, C$, and $D$, respectively. The two unlabeled holes were used for the load pins to apply the load in force $\pm F$. The following three challenge questions were used for predictive simulations:

1. What is the load force and the COD displacement at the time of the crack initiation?

2. What is the path of crack propagation?

3. At what force and COD displacement does the crack reinitiate out of the first hole, if the crack does propagate to either holes $B, C$, or $D$ ?

The teams had to answers these questions with their respective models. Team 9 from the University of Arizona used

\footnotetext{
$\overline{14}$ https://docs.scipy.org/doc/scipy-0.15.1/reference/generated/scipy. stats.linregress.html.
} 


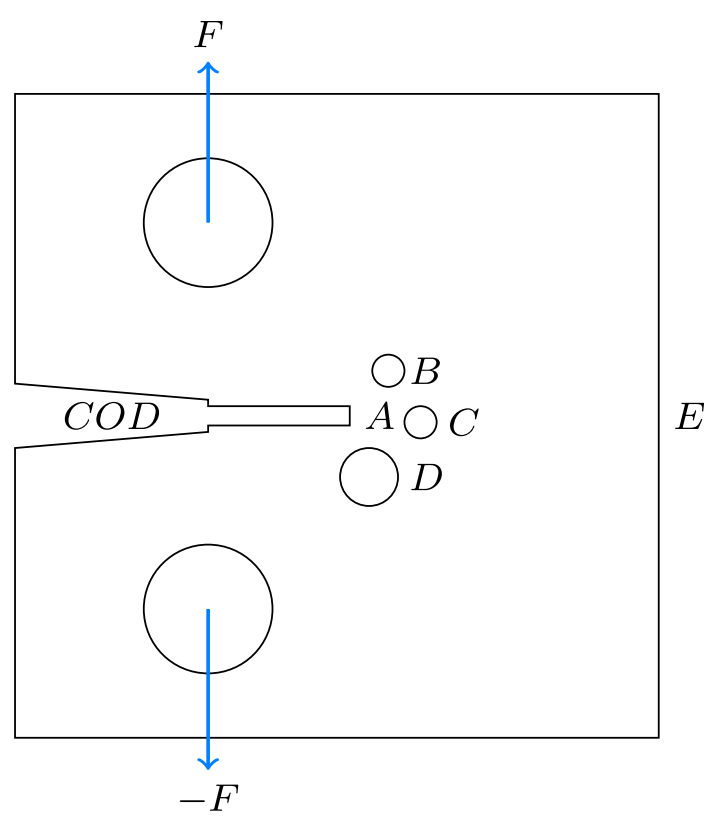

Fig. 7 First Sandia Fracture Challenge: Simplified geometry of the CT specimen to sketch the experimental quantity of interests which the peridynamic simulation was compared against. Adapted from [369]

a bond-based peridynamic model $[37,38]$ to answer these questions. The geometry was discretized using hexahedron regions with edge length of $0.63 \mathrm{~mm}$ and the horizon was $\delta=1,5621 \mathrm{~mm}$. For more details about the simulations, we refer to [369, Section 8.9].

During the ten experiments, the crack path $A-D-C-E$ occurred nine times and the crack path $A-C-E$ occurred one time. Team 9 predicted the second path in their simulations as the answer to the second question. Table 3 shows the answers to the remaining questions. The first row shows the average value for the force $(\mathrm{N})$ and the crack open displacement (COD) $((\mathrm{mm}))$ for the first crack event and the second crack event. The first value in every column is the value obtained by the load drop, and the second one the visual obtained value. The second row contains the average value obtained by the simulations of team 9 . The relative error $\epsilon_{\text {rel }}$ for the 1st crack events are for the force $-0.4 /-0.28$ and for the COD $-0.7 /-0.7$ respectively. The relative error for the 2 nd crack events are for the force $-0.31 /-0.2$ and for the COD $-0.7 /-0.7$ respectively.

Using phase-field modeling, recent results were reported in [370]. The discretization is based on tetrahedral elements with locally pre-refined meshes. The authors report force-displacements curves for different numerical models and show contour plots for three different loadings of the crack path, elastic energy, plastic energy, and the coalescence degradation function.
Table 3 The average values of the force $(\mathrm{N})$ and the crack opening displacement (COD) (mm) for the crack path $A-C-E$, see Fig. 7

\begin{tabular}{lllll}
\hline & \multicolumn{2}{l}{ 1st crack event } & \multicolumn{2}{l}{ 2nd crack event } \\
\hline & Force $(\mathrm{N})$ & COD $(\mathrm{mm})$ & Force $(\mathrm{N})$ & COD $(\mathrm{mm})$ \\
Exp & $8066 / 6621$ & $3.542 / 3.538$ & $5128 / 4363$ & $5.217 / 5.362$ \\
PD & 4782 & 1.092 & 3514 & 1.575 \\
\hline
\end{tabular}

For the experiments, the average value obtained by the load drop is shown first and the visual obtained value, second. For the simulations of team 9, their obtained average value is shown. Adapted from [369]

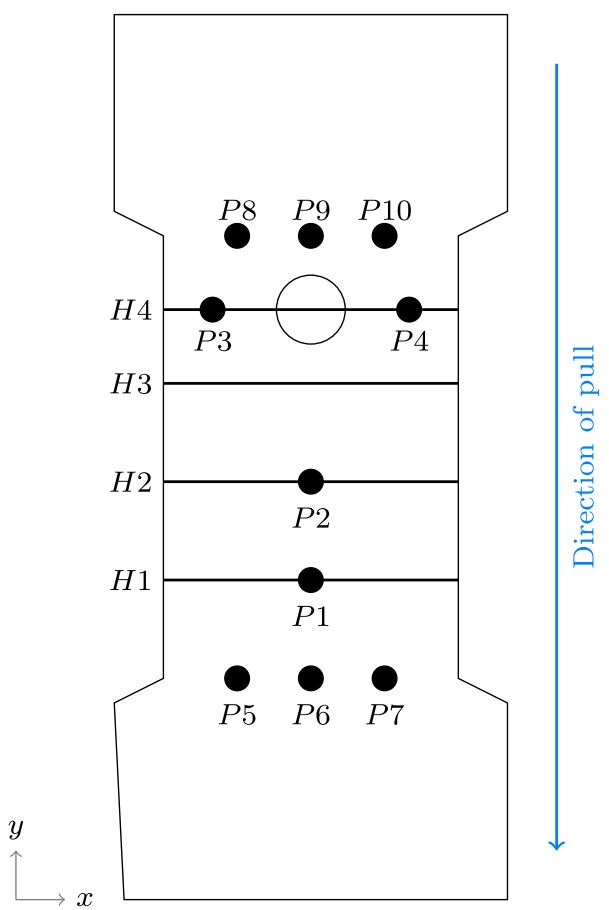

Fig. 8 Third Sandia Fracture Challenge: Simplified sketch of the geometry to showcase the quantity of interests in the fracture challenge. Adapted from [372]

\subsubsection{Third Sandia Fracture Challenge}

In the third Sandia Fracture Challenge, the predictions of ductile fracture in additively manufactured metals were studied. The data of tensile tests was provided to calibrate the simulation models. Figure 8 shows a simplified sketch of the geometry to showcase the following challenge questions for predictive simulations:

1. What is the force at the displacements $0.25,0.5,0.75$, and $1.0 \mathrm{~mm}$ ?

2. What is the force and Hencky (logarithmic) strain in the vertical direction of the points $P 1-P 4$ on the surface at the following forces: $75 \%$ and $90 \%$ of peak load (before peak), at peak load, and $90 \%$ after the peak load?

3. What is the force versus the gauge displacement for the test? 
4. What is the force and Hencky (logarithmic) strain in the vertical direction of the points $P 1-P 4$ on the surface over time?

5. What is the force and Hencky (logarithmic) strain in the vertical direction of the lines $H 1-H 4$ on the surface at the same forces as in questions 2 ?

Team C from the University of Texas Austin used an explicit peridynamic model with bond damage [371]. For the damage evolution, the Johnson-Cook model was used. The geometry was discretized with a nodal spacing of $h=0.14 \mathrm{~mm}$ resulting in a total of 460.000 nodes. Information of the used horizon were not reported in [372, Table 9]. The answers to question 1 are shown in Table 4. The relative errors are: $-0.05,-0.15,-0.47,-0.66$, respectively. The $R^{2}$ correlation for question 3 , load $(\mathrm{kN})$ vs displacement $(\mathrm{mm})$ is 0.7 . For the relative errors with respect to question 2, one can only look at the trend at the peak load, since all other loads were defined relatively to it. For the peak load, a relative error of -0.08 was reported. The relative errors for the vertical logarithmic strain (\%) for point $P 2$ are $7,4.8,-0.8$, and -0.8 , respectively. The $R^{2}$ correlation at the peak load for the Hencky strain on lines $H 3$ for question 4 is 0.44 . Unfortunately, we had issues extracting the $R^{2}$ correlations for line $H 4$ with our tools. Note that this was a blind verification, and the same team performed a revisited simulation with more details, receiving better results [373]. However, phase-field simulations were done only qualitatively using the geometry of the third Sandia Fracture Challenge [374].

\subsection{Comparison of both models with the same experimental data}

First, finite elastic deformation and rupture in rubber-like materials [375] was studied for phase-field models in [376] and for peridynamic models in [377]. In these publications, a rubber sheet with double edge notches was studied on the geometry shown in Fig. $9(80 \mathrm{~mm} \times 200 \mathrm{~mm})$ and a thickness of $3 \mathrm{~mm}$. For the experimental setup, the length of the notches $a$ varied from, $12 \mathrm{~mm}, 16 \mathrm{~mm}, 20 \mathrm{~mm}$, and $24 \mathrm{~mm}$. For the PD simulations, the horizon $\delta=3.015 h$ was used. In total, 16000 discrete PD nodes with a surrounding volume of $\mathrm{V}=1 \mathrm{~mm}^{2}$ and a nodal spacing of $h=1 \mathrm{~mm}$. For the PF sim-

Table 4 Comparison of the measurements and the obtained loads in the simulations for four different displacements

\begin{tabular}{lllll}
\hline \multicolumn{5}{l}{ Force $(\mathrm{kN})$ for four displacements $(\mathrm{mm})$} \\
\hline & 0.25 & 0.5 & 0.75 & 1.0 \\
\hline Exp & 7.884 & 8.164 & 8.203 & 6.538 \\
PD & 7.469 & 6.919 & 4.330 & 2.188
\end{tabular}

For the comparison, the nominal load is considered. Adapted from [372] ulations, a quad mesh with a resolution of $h=6.66 \mathrm{~mm}$ was used. The applied displacement $(\mathrm{mm})$ vs the reaction force (N) was compared against the experimental observations and the corresponding simulation results. The $R^{2}$ correlation for PD are: $0.83,0.99,0.98,0.98,0.98$, and 0.78 respectively. The $R^{2}$ correlation for $\mathrm{PF}$ are: $0.78,0.84,1,0.64$, and 0.65 respectively.

Second, dynamic brittle fracture in glassy materials was studied in [378,379]. In this study, a phase-field model [186], a discontinuous-Galerkin implementation of $\mathrm{PD}$ [380], and a meshfree discretization of PD [38] are used in the geometry shown in Fig. 10. For the PD simulation, a nodal spacing $h=0.1 \mathrm{~mm}$ and a horizon $\delta=0.5 \mathrm{~mm}$ were used. For the discontinuous-Galerkin implementation of PD a nonuniform mesh with average element size $h$ of $0.1 \mathrm{~mm}$ and a horizon $\delta$ of $0.5 \mathrm{~mm}$ was used. For the phase-field model, the nodal spacing was $h=0.3 \mathrm{~mm}$ and the length scale parameter $l_{0}$ was $0.6 \mathrm{~mm}$. Note that the authors did some $\delta$-convergence study in [379], however, we only report the finest resolution here. Fore more details, we refer to [379, Section 5.2]. For all three implementations, the crack angle after branching, the time of crack branching, and position of the crack branching were compared with the experimental results. In this study various discretization parameters were studied, however, we report the discretization parameters corresponding to the best agreement with the experimental data. First, the value for the meshfree discretization is presented, followed by the value for the discontinuous-Galerkin discretization, and the value for the phase-field model last. The relative errors for the crack angle are: $-0.21,-0.35$, and -0.51 , respectively. The relative errors -0.06 for the event of crack branching in time are the same for all simulations. The relative errors for the crack branching position are: $0,-0.12$, and 0 , respectively.

\section{Comparison between peridynamics and phase-field fracture models}

In this section, the two approaches PD and PF are compared with respect to their computational aspects, advantages in simulating complex fracture phenomena, and the challenges faced by these numerical methods. For peridynamics, we assume that the presented aspects hold for all three models presented in Fig. 2. If one aspect holds only for specific models, we will mention that explicitly in the text below.

\subsection{Computational aspects}

In this section, we focus on the computational aspects of both models from a bird's eye view and compare the computations on a very high-level. To do so, we define the quantity of a field which can be a vector field $\mathbf{f}=\left\{f_{1}, \ldots, f_{n} \mid f_{i} \in \mathbb{R}^{3}\right\}$ or a scalar field $f=\left\{f_{1}, \ldots, f_{n} \mid f_{i} \in \mathbb{R}\right\}$. Peridynamics is a 
Table 5 Overview of the Sandia Fracture Challenge with contributions of peridynamic models. Two different research groups contributed to the first and second Sandia Fracture Challenge using a peridynamic model. To the best knowledge of the authors, no phase-field model contributed to the Sandia Fracture Challenge. However, phase-field simulations were done using the geometry of the third Sandia Fracture Challenge [374]. To compare with the experimental measurement, the relative error is provided for scalar values, and the $R^{2}$ correlation for a series of values. For the first Sandia Fracture Challenge, the following quantities were studied: $(a)$ Force $(\mathrm{N}) 1$ st crack event, $(b)$ Crack Opening Displacement (COD) (mm) 1st crack event, (c) Force (N) 2nd crack event, and $(d)$ COD $(\mathrm{mm})$ 2nd crack event. For the third Sandia Fracture Challenge, the following quantities were studied: $(a-d)$ Force $(\mathrm{kN})$ at $0.25,0.5,0.75$, and $1 \mathrm{~mm}$ displacement; (e) Force vs displacement (time series); $(f)$ Force $(\mathrm{kN})$ at Peak load; and $(g)$ Hencky strain (\%) on line $H 3$ at peak load

\begin{tabular}{|c|c|c|c|c|c|c|c|c|c|c|c|}
\hline & \multicolumn{4}{|c|}{ First Sandia Fracture Challenge [369] } & \multicolumn{7}{|c|}{ Third Sandia Fracture Challenge [372] } \\
\hline & a & $\mathrm{b}$ & $\mathrm{c}$ & $\mathrm{d}$ & a & $\mathrm{b}$ & $\mathrm{c}$ & $\mathrm{d}$ & e & $\mathrm{f}$ & $\mathrm{g}$ \\
\hline$\epsilon_{\mathrm{rel}}$ & $-0.4 /-0.28$ & -0.7 & $-0.31 /-0.2$ & -0.7 & -0.05 & -0.15 & -0.47 & -0.66 & & 0.08 & \\
\hline$R^{2}$ & & & & & & & & & 0.7 & & 0.44 \\
\hline
\end{tabular}

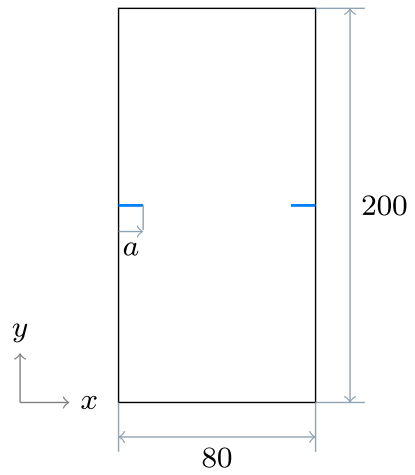

Fig. 9 Geometry of the rubber sheet $(80 \mathrm{~mm} \times 200 \mathrm{~mm})$ and a thickness of $3 \mathrm{~mm}$. The length $a$ of the notches varied from, $12 \mathrm{~mm}, 16 \mathrm{~mm}, 20 \mathrm{~mm}$, and $24 \mathrm{~mm}$. The radius of the notches is fixed at $1 \mathrm{~mm}$

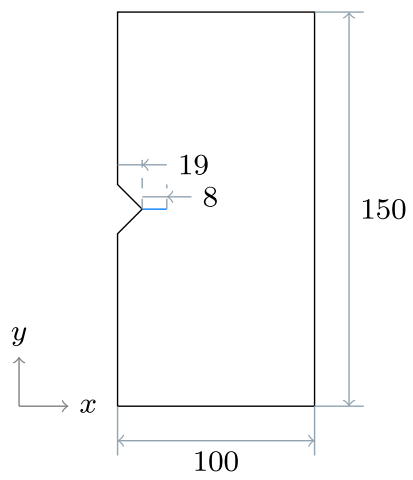

Fig. 10 Sketch of the geometry $(100 \mathrm{~mm} \times 150 \mathrm{~mm})$ with a thickness of $\mathrm{mm}$. The angle of the cut-off is $40^{\circ}$ and the initial crack has a length of $8 \mathrm{~mm}$

single-field model, here one just solves for the displacement field $\mathbf{u}$ and the peridynamic damage field $d(\mathbf{u})$ is obtained from the displacement field using the constitutive law. The displacement field is solved with explicit or implicit time integration [381-385]. The majority of PD simulations utilized bond-based models due to the increased computational costs for the state-based models. Similarly, the majority of simulations utilized explicit time integration due to their lower computational costs.
For phase-field, we have a two field model with the displacement field $\mathbf{u}$ and the damage field $\varphi$.

For staggered schemes and alternating minimization [186, $187,222,296,300-302,386]$ the global system is decoupled, first, one solves for the displacement field $\mathbf{u}$ and second, one solves for the phase-field damage field $\varphi$ independently. For the equation of motion, implicit or explicit time integration schemes can be utilized. For the monolithic scheme [151,155,236,304,306,307,319,353,387] the displacement field and the phase-field damage field are fully coupled and solved simultaneously. Pham et al. [162] suggested that a suitable choice of fracture process zone corresponding to the intrinsic length scale associated with the phase-field model could provide valid predictions of crack growth in quasi-static brittle fracture.

Classical elasticity parameters can be used to calibrate both models. Both models require a minimal set of parameters for calibration, i.e. Young's modulus $E$, Poisson's ratio $v$, and fracture energy $G_{c}$, which all can be experimentally determined. Thus, both models could use the same elasticity and fracture properties obtained by an experiment to calibrate and validate against the same quantity of interest. Both models depend on length scale parameters; $l_{0}$ for phase-field models and the horizon $\delta$ for peridynamic models needs to be calibrated. Techniques for calibration that include material strength and flaw size have been shown for PF [388] and PD $[55,115]$. On the other hand, when sharp cracks approximations are needed, mathematically $l_{0}$ should tend to zero (see Sect. 2.2), as confirmed for PF with numerical simulations in [311] using an academic test case in which manufactured solutions for the crack opening displacement and total crack volume were constructed [389]. In the case of PD with bond softening, one sees that the damage is confined to a thin zone about the crack line of thickness controlled by the PD horizon $\delta$, [128]. Here, the thickness is $\delta+2 h$ where the mesh diameter $h$ is $h=o(\delta)$. Goswami et al. [390] developed an enhanced physics-informed neural network (PINN) based machine learning (ML) for the fracture growth and propagation problem using PF. Nguyen et al. [391] used 
ML to develop relationships between the displacements of a material point and the displacements of its neighbors and the applied forces within PD framework. Mandal [392] presented a comparison of PF and PD models and [393] for a tensile impulse traction on the pre-crack faces experiment. Good agreements were reported for crack tip velocities for no-branching case as well as branching for higher stress intensities cases despite the post-processing of PD and PF results using distinct algorithms.

We conclude that there are very few results available where both models are compared against the same experimental data. Confronted with this paucity of information it is difficult to get insights regarding better suitability of a method for specific crack phenomena. With this in mind, the Sandia Fracture Challenge is pointed out in this review for the two main reasons described in Sect. 4.1. This makes the Sandia Fracture Challenge an excellent problem to benchmark phase-field models and peridynamic models in order to assess their performances for different kinds of fracture phenomena. Further, it would be a good exercise for both communities to come together and define a common set of benchmark simulations for experimental validation and model performance assessment.

\subsection{Advantages}

Several advantages are highlighted for both PD and PF approaches to show why these two methods have been popular approaches to understand fracture phenomena.

1. Crack initiation: Growth at the tips of long preexisting cracks are handled by both phase field and peridynamics noting that brittle crack extension is energy based. For short interior cracks and notches quasi-static phase field models have introduced strength based driving forces for to account for crack nucleation Kumar et al. [175]. For peridynamics crack nucleation about defects are manifested as dynamic instabilities Silling et al. [394], for cohesive models see Lipton et al. [47], [124].

2. Notion of damage in the model representation: In most other models or computational techniques an additional criteria, e.g. as in Linear Elastic Fracture Mechanics, is needed to describe the growth of cracks. However, in peridynamic and phase-field models the criteria for the crack growth is determined as a part of the solution and no external criteria is needed. The PD correspondence models offer the opportunity to incorporate classic continuum damage models in state based peridynamics see Tupek et al. [395].

3. Increasing complexity in multi-field fracture: Both models were extended to multi-field fracture. For peridynamic models: thermal effects [396-400], diffusion
[113,397,401-403], geomechanical fracture [1,404-414], and corrosion [415-417]. For phase-field simulations: hydraulic fracture [168,201-203,293,355,357,360,418428], diffusion [209], thermo-elastic-plastic [199], thermal effects $[152,197,198,286,429]$, geologic/geomaterials [246,258,430], and fluid-structure interaction $[17,347,431]$.

\subsection{Challenges}

The following important issues are identified as challenges in the context of both PD and PF modeling.

\section{Common challenges to PD and PF}

1. From dynamic to quasi-static evolution A long range goal for fracture modelling will be the ability to recover quasistatic fracture models directly from dynamic fracture models without ad-hoc assumptions. This aspect is largely absent in both PF and PD approaches. To the best of our knowledge such questions have been raised and partially answered only recently for a new local fracture model in the context of the dynamic peeling test in one dimension as described in Freund [432]. In this context the dynamic model is a local model for free de-cohesion developed in Dal Maso et al. [433] and the quasistatic limit is identified in Lazzarioni and Nardini [434]. It is recognized that this is a hard problem and presents a challenge for PD and PF.

2. Computational cost: Both PD and PF approaches are computationally expensive. For peridynamics it is the meshless discretization, which is computational intensive, similar to molecular dynamics (MD) and smoothedparticle hydrodynamics (SPH). To accelerate the computations implementations using the Message Passing Interface (MPI) [84,86,87], the C++ standard library for parallelism and concurrency (HPX) [88], and GPU accelerated [93-95] are available. To speed up the implicit time integration the following methods were proposed: Finite element approaches (FEM) [80,82,85], a Galerkin method that exploits the matrix structure [435], using sparse matrices instead of a dense tangent stiffness matrix [436], adaptive dynamic relaxation schemes (ADR) [437439], the Fire algorithm [440,441], an explicit tangent stiffness matrix [442] using bond softening [46], a convolution based approach $[443,444]$, and the GMRES algorithm $[444,445]$ in conjunction with the Arnoldi process [446,447]. Here, bond-based material models are computationally cheaper than state-based models. Furthermore explicit time integration is computationally cheaper than implicit time integration.

For phase-field models the length scale parameter $l_{0}$ tends to become small, thus, requiring small mesh sizes 
for finite element discretizations. Therefore, the method gets computationally expensive due to the large number of mesh elements. Phase-field models could be accelerated using a staggered schemes instead of a monolithic scheme [226], GPU acceleration [331], and the Message Passing Interface (MPI) based parallelization [237,311], and matrix-free geometric multigrid methods [307,312]. Other attempts to reduce the computational costs are model order reduction [448,449], sympletic time integrators [450], adaptive schemes [187,202,290,331,333, $336,337,451]$, and global/local (multiscale) approaches [315,349,351,452].

3. Lack of detailed three-dimensional simulations: Probably due to their computational expenses, only a few threedimensional simulations using PF and PD are reviewed here. Following three-dimensional PD simulations are available: hydraulic fracture [410,453], the Brokenshire torsion experiment [454], polymer chains [455], Kalthoff Winkler [143], pitting corrosion damage [456], 3-point bending [96], impact damage on the glass layered structure $[96,457,458]$, penny-shaped crack in a cylindrical bar [459], double edge notch specimen [459], ductile material behavior in a rectangular bar [460], pressurized cylinder [222], and reinforced concrete lap splice [56].

The following three-dimensional research studies using PF are available: formation and growth of echelon cracks [228], pressure vessel simulation [222], singleedge notched shear test [226], cube with rigid spherical inclusion under tension [221], a block-like geometry [342], Kalthoff Winkler experiment [221], bolted plate compared against experimental results [225], simple shear tests of thoracic aorta with anisotropic failure compared against experimental results [227], random nucleation sites [223], L-shaped specimen [224,307], tension test of cube with spherical inclusion [151], bending of Hopkinson bar [461], and Sneddon/Lowengrub benchmark [202,287,311], and non-isothermal pressurized fractures [462].

To list some representative three-dimensional simulations, Weinberg et al. [463] showed stress distributions for modes I, II, and III fractures using NURBS-based finite elements in three-dimensional simulations. Heider et al. (2018) compared the hydraulic fracturing simulation results using $\mathrm{PF}$ against the experimental data for granite samples from the "Hohenberg" quarry in Germany with good predictive accuracy (within experimental relative errors less than $15 \%$ ) for the pressure needed to initiate the crack in both $2 \mathrm{D}$ and $3 \mathrm{D}$ geometries. Another plausible reason for the lack of detailed three-dimensional fracture simulations could also be the associated challenges to perform detailed three-dimensional experimental measurements and provide validation data sets.
4. Extraction of crack tip/surfaces: Since both models have a notion of damage, the so-called phase-field crack function $\varphi$ and the peridynamic damage parameter $d$, the position of the crack tip/surface is not encoded in the model and needs to be approximated. This phenomenon is not limited to phase-field [281] and peridynamic models, e.g. $[464,465]$, and relates to any other method which does not have explicit crack representation in the model. This could be a source of error for tracking the crack tip and comparing the crack tip velocity against experimental observations in dynamic fracture simulations. Ziaei-Rad et al. (2016) used the non-maximum suppression technique from an image processing field to detect the ridge of the phase-field profile and then applied cubic spline fit to determine the crack path representation with reasonable success to identify crack branching as well as crack tips within the mesh resolution limits. Agrawal and Dayal [466] partially explained the relationship between phasefield and crack opening displacement and irreversibility in the phase-field model. Yoshioka et al. [467] presented two approaches - a line integral and a level-set method, to compute the crack opening displacement that is required in hydraulic fracturing simulations and demonstrated that both approaches computed the crack opening temporal growth accurately. Despite these recent advances, there is still room for improvement to extract the complex multiple interacting crack surfaces from simulations.

5. Lack of validation studies against available experimental data: Validation against experimental data for peridynamics is summarized in [4] and for phase-field models in [16, Section 2.12]. However, for an accurate comparison of these two models, the same experiment or a set of experiments should be utilized to gain some insights of both methods on the same problem. Table 6 lists the phase-field and peridynamic models which were compared against the same experimental data. On the other hand, accessing raw experimental data is a different challenge [366], and one of the Sandia Fracture Challenges could be used to validate peridynamics and phase-field models against the same experimental data.

6. Unavailability in commercial codes: Most simulations of PF and PD models use their implementations in corresponding scientific code bases. At the time of writing of this review, not many commercial codes implemented either one of the models. LS-DYNA provides a bondbased peridynamics implementation discretized with the discontinuous Galerkin FEM [96].

7. Crack nucleation: Although crack propagation and path is autonomously handled by both models, a complete theoretical understanding of nucleation for dynamic and quasistatic fracture is incomplete.

Recent developments include the introduction of a strength based crack driving force for quasistatic PD [175] 
based on a realistic phenomenology and comparison with experiments. [162,468-471]. As expected it is a very active area of interest both for PF [175,176,388,472] and PD [473-475].

8. Incompressible hyper elastic material behavior: At the time of completing this review, not many material models or simulations for incompressible hyper elastic material behavior, e.g. a Poisson ratio $v=0.5$, were available for $\mathrm{PF}[283,284]$ and the recent $\mathrm{PhD}$ thesis [476], and the application to carbon black filled ethylene propylene diene monomer rubber (EPDM) was done in [285]. For PD we have $[140,477,478]$. Note that modeling of hyper elastic material behavior is challenging for any numerical method since the constitutive material law must reflect material behaviors such as a neo-Hookean [479] or Mooney-Rivlin [480] solids and requires for the wellposedness the inf-sup condition for both the continuous problem formulation and the discretization.

9. Microscale view of crack propagation physics using molecular dynamics (MD) simulations:

Seleson et al. [130] showed that peridynamics (PD) models can recover the same dynamics as the MD model through appropriate selection of the length scale for smooth deformations. Ahadi and Melin [481] investigated accuracy of PD in capturing features emerging from atomistic simulation [482] through calibration of interparticle bond strength and length scale parameters elastic plastic effects. In a similar attempt to connect the phase-field method to MD, Patil et al. [483] derived PFM parameters from the MD atomistic simulations and showed that the theoretical energy release rate $G$ and internal length parameter are consistent with the MD simulation results. It is important to note that the microscale physics of crack nucleation and growth through MD atomistic simulation can provide the information for upscaling [484]. Given the current state of the art, the relation between first principles models and the macroscopic models of PD and PF with crack nucleation and propagation has not been firmly established in the literature.

\section{Specific challenges for peridynamics fracture models}

The following challenges are highlighted to show the difficulties in applying boundary conditions, specific material models, and controlling numerical errors in PD.

1. Application of boundary conditions: As mentioned in [4] a major challenge within PD is the treatment of boundary conditions in a non-local fashion [485-490]. This is seen in nonlocal traction conditions for state based linear peridynamic solids where surface effects appear. For this case techniques to handle nonlocal traction are recently developed [127]. More general non-ordinary state based and correspondence models have yet to be addressed. For local to nonlocal coupling one approach is to couple local and non-local models to enforce boundary conditions in the local region and have the non-local model in the region where cracks and fractures arise. For more details, we refer to the review on non-local coupling approaches [491].

2. Constitutive modeling Figure 2 illustrates the plethora of material models proposed for peridynamics. The question of choosing a nonlocal model that is an acceptable representative for a particular material system must be the focus of a coordinated theoretical and experimental effort. This applies to bond based and state based PD models as well as correspondence models Tupek et al. [395] that permit the usage of constitutive models from local theory within the peridynamic formulation. [41,41,63-70,72-78,492-494]. The possibility for zero energy modes in correspondence models has been acknowledged and methods for stabilization are being developed [492,493,495].

3. Choice of discretization parameters: As mentioned in [4] the choice of the nodal spacing and the horizon results in diverse convergence scenarios $[111,114]$. One challenge is to find the proper ratio between the horizon and mesh size, since the simulations are sensitive $[115,116]$ with respect of these parameters. One adjustment is to select the ratio such that the PD simulation matches the dispersion curve obtained by the experiment [496]. Another adjustment is to determine the horizon by Griffith's brittle failure criterion [55]. To determine the discretization parameters from experimental data, the peridynamic formulation of the virtual field method could be applied [497].

4. Surface effects and corrections: Most PD models are calibrated under the assumption that a discrete PD node has a full neighborhood. However, points close to the boundary do not have a full neighborhood and their material properties are slightly different from the ones with the full neighborhood. This is the so-called surface effect. Le and Bobaru studied some proposed methods/algorithms to address the surface effect [498]. The following methods/algorithms are available: volume method [9, Section 2], force density method [499], energy density method $[10,499,500]$, force normalization method [82], a modified position-aware linear solid constitutive model [72], fictitious node method [397,501], and correction of the transient heat conduction [502].

5. Development of adaptive PD models and methods: There is a lack of adaptive methods developed to handle peridynamic fracture problems for coupling interior dynamics to boundary effects and for coupling inelastic to elastic domains within a simulation. A variable horizon 
Table 6 For the two experiments, phase-field and peridynamic models were used for comparison

\begin{tabular}{|c|c|c|c|c|c|c|c|c|c|}
\hline \multicolumn{6}{|c|}{ Double edge notches } & \multicolumn{4}{|c|}{ Dynamic brittle fracture in glassy materials } \\
\hline$\overline{R^{2}}$ & a & $\mathrm{b}$ & $\mathrm{c}$ & $\mathrm{d}$ & $\mathrm{e}$ & $\epsilon_{\text {rel }}$ & a & $\mathrm{b}$ & $\mathrm{c}$ \\
\hline PD [377] & 0.83 & 0.99 & 0.98 & 0.98 & 0.78 & PD [379] & $-0.21 /-0.35$ & $0 /-0.12$ & $-0.06 /-0.06$ \\
\hline PF [376] & 0.78 & 0.84 & 1 & 0.64 & 0.65 & $\mathrm{PF}$ [379] & -0.51 & 0 & -0.06 \\
\hline EXP [375] & - & - & - & - & - & EXP [378] & $57^{\circ} / 55^{\circ}$ & $\{0.53,0.57,0.88\}$ Width & $30.7 \pm 1.5 \mu \mathrm{s}$ \\
\hline
\end{tabular}

To compare with the experimental measurement, the relative error is provided for scalar values and the $R^{2}$ correlation for a series of values. For the double edge notches, the displacement $(\mathrm{mm})$ vs the reaction force $(\mathrm{N})$ was compared for a initial crack lengths $12,16,20,24$, and 28mm, see $(a)-(d)$. For the dynamic brittle fracture in glassy materials, the following quantities were studied: $(a)$ crack angle, $(b)$ crack branching position, and $(c)$ crack branching event. Here, two different PD discretizations: a meshfree discretion [38] and a discontinuous-Galerkin implementation [380]. For each error measurement, the first value is with respect to the meshfree discretization, and the second one with respect to the discontinuous-Galerkin implementation

approach is proposed for adaptivity in [503] and [504]. It is well known that nonlocal models are far more expensive to simulate than local models. Additionally they can induce artificial dispersive artifacts in otherwise local elastic regions. These considerations provide motivation for adaptive local-nonlocal models for fracture evolution. Here the fracture set is evolved in terms of bond based PD while FEM methods can be used away from the fracture set [505]. More on this type of numerical modeling and implementation can be found in [491].

6. Asymptotically compatible quadrature methods: An additional way to control accuracy of peridynamic methods is through development of asymptotically compatible quadrature methods for state based fracture as in recent approach of [127]. Here, the numerical scheme is designed to recover linear elastic behavior away from the crack set asymptotically as the horizon tends to zero.

7. Ductile fracture: As of the time of writing this review, not many material models and simulation for ductile fracture were available [506-509]. Note that ductile tearing is challenging for any numerical method, due to the choice of an appropriate ductile failure model. This failure model needs to incorporate the failure of hydrostatic stress (or triaxiality of stress) to predict ductile failure. Here, state-based and correspondence models will be beneficial, as bond-based material models are limited to model two point interactions.

8. Opportunities for quasistatic PD models: The preponderance of peridynamic simulation has focused on dynamic problems and this provides an opportunity for quasistatic fracture modeling [382,510-512] with suitable PD models. There are plenty of experimental benchmarks for the validation of peridynamic models in the quasistatic regime.

\section{Specific challenges for phase-field fracture models}

The following challenges are highlighted regarding the handling of complex geometries, material models, and controlling numerical errors in estimating crack surface geometries in $\mathrm{PF}$.

1. Crack path intersecting holes, obstacles, and boundaries: Several issues were reported while obtaining crack paths in agreement with LEFM for problems involving holes [16]. Another study [163] concluded that judgement on if a crack arrests or the method simply does not permit continuation across obstacles, requires expert knowledge. In pressurized fractures, see e.g., [306], the fracture often branches, which raises however whether this is physically reasonable. Moreover, goal functional evaluations are sensitive to boundary conditions and the domain size [311].

2. Fast crack propagation under dynamic loading: For some fast crack propagation simulations, e.g. [466], the calculated fracture velocity overestimated the fracture energy dissipation.

3. Composite/Concrete fracture: At the time of writing this review, not many fracture/damage models for composites [267,513,514] and concrete [515-518] were available.

4. Asymptotic computational understanding of the interaction of regularization, model, and discretization parameters: In terms of numerical and computational convergence analysis, current PF understanding is still incomplete. Ingredients of numerical analysis from image segmentation [519], phase-field in fluid flow [520,521] are available. Furthermore, computational convergences analyses for phase-field fracture have been undertaken $[17,290]$ and $[522,523]$. Such a rigorous numerical analysis for a phase-field fracture model substantiated with numerical tests is missing to date. However, passing to 
the limits in the regularization parameters also requires to change the mathematical function spaces, e.g., [147,148].

5. Robust and efficient linear solvers and preconditioners Closely related to the previous aspect is the development of iterative or multigrid linear solvers and preconditioners. Several suggestions exist as we have previously discussed, but a robust preconditioner for variations in the length-scale $l_{0}$, the regularization parameter $\kappa$, both spatial and temporal discretization parameters, and material parameter limits, for instance, Poisson's ratio $v \rightarrow 0.5$ has not yet been fully understood; first attempts can be found in the PhD theses [524] and [476]. The challenge are spectral-equivalent operators for preconditioning and the multiscale-multidomain character due to the variations of several orders of magnitude of the regularization parameters.

This review paper has highlighted some common challenges and specific challenges for both models. In our opinion, listing these challenges are useful in many ways. First, these challenges can guide young researchers to interesting research topics they might address for their future career. Second, these challenges need to be addressed for rigorous comparison against experiments. Notably in Sect. 5 we point out that there are few comparisons of both methods against the same experiment. Here, more work is needed for comparison of both models' accuracy for specific crack and fracture phenomena. Another aspect is that both models have not participated in the Sandia Fracture Challenge as consistently as other methods. Third, more rigorous mathematical and numerical analysis of cracks speeds, locations and bifurcations are needed for both methods. Application and calibration of these methods to composite materials should be carried out. We believe that this is necessary for both methods to have a broader acceptance in industry. Presently these methods are being applied by some research and development departments of companies, however, they are not used for validation and certification of products. This is in contrast with the finite element method where it is used in the final structural certification of the the Boeing 777 . Here, one could argue that the finite element method was introduced 75 years ago [525-527], while free fracture methods were introduced only around 21 years ago. However, addressing these challenges is crucial for a broader adaption of these methods outside the academic setting.

\section{Conclusions}

A comprehensive review of two numerical modeling approaches - Peridynamics (PD) and Phase-field (PF) is presented with the expectation to highlight their advantages, as well as challenges in modeling fracture initiation, propagation, and predictive capabilities for experimental validation. Both numerical methods can retrieve a consistent microscale physics of crack initiation and propagation. Despite both approaches being computationally challenging, their advantages in capturing multiple fracture interactions with minimal amount of phenomenological assumptions and closures make $\mathrm{PD}$ and PF as a good choice to understand engineering fracture mechanics. The following items are listed here for further improvement of both modeling approaches:

- Both PD and PF need to be evaluated against the same experimental benchmark for a reasonable comparison in a blind validation manner. Several experimental datasets are identified as the available community resources.

- Phase-field simulation results for the Sandia Fracture Challenge problems could provide the missing piece of information for a comprehensive and validated comparison among the two modeling approaches.

- There is in general a lack of comparative studies between these two leading modeling approaches for fracture initiation and propagation, even for the same set of simple fracture experiments. ${ }^{15,16}$

Acknowledgements This work was partly funded by DTIC Contract FA8075-14-D-0002/0007 and the Center of Computation \& Technology at Louisiana State University. Authors also thank Dr. Stewart Silling for his comments on the manuscript prior to the submission and the participants of the Workshop on Experimental and Computational Fracture Mechanics ${ }^{1} 5$ and of the Banff International Research Station: Hydraulic Fracturing: Modeling, Simulation, and Experiment ${ }^{1} 6$ for the discussion on peridynamics and phase-field models which provided several ideas for the challenging applications. In addition, we thank Haim Waismann, John Dolbow, Florin Bobaru, Steve Sun, Jinhyun Choo, and Masoud Behzadinasab for their remarks on the preprint. This material is partially based upon work supported by the U. S. Army Research Laboratory and the U. S. Army Research Office under Contract/Grant Number W911NF1610456. TW was partially funded by the the German Research Foundation, Priority Program 1748 (DFG SPP 1748) under the grant number WI4367/2-1 (project number 392587580).

Funding Open Access funding enabled and organized by Projekt DEAL.

Open Access This article is licensed under a Creative Commons Attribution 4.0 International License, which permits use, sharing, adaptation, distribution and reproduction in any medium or format, as long as you give appropriate credit to the original author(s) and the source, provide a link to the Creative Commons licence, and indicate if changes were made. The images or other third party material in this article are included in the article's Creative Commons licence, unless indicated otherwise in a credit line to the material. If material is not included in the article's Creative Commons licence and your intended use is not permitted by statutory regulation or exceeds the permitted use, you will need to obtain permission directly from the copy-

\footnotetext{
15 Playlist (WFM2020).

16 Playlist (Hydraulic Fracturing: Modeling, Simulation, and Experiment).
} 
right holder. To view a copy of this licence, visit http://creativecomm ons.org/licenses/by/4.0/.

\section{References}

1. Hattori G, Trevelyan J, Augarde CE, Coombs WM, Aplin AC (2017) Numerical simulation of fracking in shale rocks: current state and future approaches. Arch Comput Method Eng 24(2):281-317

2. Silling S, Wick T, Ravi-Chandar K, Guilleminot J, Dolbow J, Finberg J, Diehl P, Prudhomme S, Lipton R, Seleson P (2020) Workshop on experimental and computational fracture mechanics 2020, Tech. Rep. ORNL/TM-2020/1714, Oak Ridge National Laboratory

3. Javili A, Morasata R, Oterkus E, Oterkus S (2019) Peridynamics review. Math Mech Solids 24(11):3714-3739

4. Diehl P, Prudhomme S, Lévesque M (2019) A review of benchmark experiments for the validation of peridynamics models. $\mathrm{J}$ Peridyn Nonlocal Model 1(1):14-35

5. Isiet M, Mišković I, Mišković S (2021) Review of peridynamic modelling of material failure and damage due to impact. Int J Impact Eng 147:103740

6. Hattori G, Hobbs M, Orr J (2021) A review on the developments of peridynamics for reinforced concrete structures, Archives of Computational Methods in Engineering, pp 1-32

7. Zhou X-P, Wang Y-T (2021) State-of-the-art review on the progressive failure characteristics of geomaterials in peridynamic theory. J Eng Mech 147(1):03120001

8. Hidayat MIP, Lemma TA, Machmudah A (2021) A review on connection between meshfree peridynamics and meshfree methods. In: AIP Conference Proceedings, vol. 2384, p 030006, AIP Publishing LL

9. Bobaru F, Foster JT, Geubelle PH, Silling SA (2016) Handbook of peridynamic modeling. CRC Press, Florida

10. Madenci E, Oterkus E (2014) Peridynamic theory. In: Theory peridynamic, applications its (eds). Springer, Berlin, pp 19-43

11. Gerstle WH (2015) Introduction to practical peridynamics: computational solid mechanics without stress and strain, vol 1. World Scientific Publishing Company, Singapore

12. Bourdin B, Francfort G, Marigo J-J (2008) The variational approach to fracture. J Elasticity 91(1-3):1-148

13. Rabczuk T (2013) Computational methods for fracture in brittle and quasi-brittle solids: State-of-the-art review and future perspectives. ISRN Appl Math 2013:38

14. Ambati M, Gerasimov T, De Lorenzis L (2015) A review on phasefield models of brittle fracture and a new fast hybrid formulation. Comput Mech 55(2):383-405

15. Bourdin B, Francfort GA (2019) Past and present of variational fracture. SIAM News 52:9

16. Wu J-Y, Nguyen VP, Nguyen CT, Sutula D, Sinaie S, Bordas SP (2020) Chapter one - phase-field modeling of fracture. In: Bordas SP, Balint DS (eds) Advances in applied mechanics, vol 53. Elsevier, Amsterdam, pp 1-183

17. Wick T (2020) Multiphysics phase-field fracture: modeling, adaptive discretizations, and solvers. De Gruyter, Berlin, Boston

18. Francfort G (2021) Variational fracture: twenty years after. International Journal of Fracture, pp 1-11

19. Crouch S (1976) Solution of plane elastic problem by the displacements discontinuity method. Int J Num Meth Eng 10:301-343

20. Xu XP, Needleman A (1994) Numerical simulations of fast crack growth in brittle solids. J Mech Phys Solids 42:1397-1434

21. Chang C, Mear ME (1995) A boundary element method for two dimensional linear elastic fracture analysis. Int J Fract 74:219-251
22. Fries T-P, Belytschko T (2010) The extended/generalized finite element method: An overview of the method and its applications. Int J Numer Meth Engrg 84:253-304

23. Strouboulis T, Babuška I, Copps K (2000) The design and analysis of the generalized finite element method. Comput Methods Appl Mech Eng 181(1):43-69

24. Moes N, Dolbow J, Belytschko T (1999) A finite element method for crack growth without remeshing. Int J Numer Meth Engrg 46:131-150

25. Stochino F, Qinami A, Kaliske M (2017) Eigenerosion for static and dynamic brittle fracture. Eng Fract Mech 182:537-551

26. Pandolfi A, Ortiz M (2012) An eigenerosion approach to brittle fracture. Int J Numer Meth Eng 92(8):694-714

27. Pandolfi A, Li B, Ortiz M (2013) Modeling fracture by materialpoint erosion. Int J Fract 184(1-2):3-16

28. Wang K, Sun W (2017) A unified variational eigen-erosion framework for interacting brittle fractures and compaction bands in fluid-infiltrating porous media. Comput Methods Appl Mech Eng 318:1-32

29. Cervera M, Barbat G, Chiumenti M, Wu J-Y (2021) A Comparative Review of XFEM, mixed FEM and Phase-Field Models for Quasi-brittle Cracking. Archives of Computational Methods in Engineering. https://doi.org/10.1007/s11831-021-09604-8

30. Alder BJ, Wainwright TE (1959) Studies in molecular dynamics. i. general method. J Chem Phys 31(2):459-466

31. Gingold RA, Monaghan JJ (1977) Smoothed particle hydrodynamics: theory and application to non-spherical stars. Mon Not R Astron Soc 181(3):375-389

32. Lucy LB (1977) A numerical approach to the testing of the fission hypothesis. Astron J 82:1013-1024

33. Cucker F, Smale S (2007) Emergent behavior in flocks. Trans Autom Control 52:852-862

34. Trygve AM, Karper K, Trivisa K (2015) Hydrodynamic limit of the kinetic cucker-smale flocking model. Mathematical Models and methods in the Applied Sciences M3AS, 25:131-163

35. Figalli A, Kang M-J (2019) A rigorous derivation from the kinetic cucker-smale model to the pressureless euler system with nonlocal alignment. Anal PDE 12(3):843-866

36. Shu R, Tadmor E (2020) Flocking hydrodynamics with external potentials. Arch Ration Mech Anal 238:347-1381

37. Silling S (2000) Reformulation of elasticity theory for discontinuities and long-range forces. J Mech Phys Solids 48(1):175-209

38. Silling SA, Askari E (2005) A meshfree method based on the peridynamic model of solid mechanics. Comput Struct 83(1718):1526-1535

39. Kunin IA (2012) Elastic media with microstructure I: onedimensional models, vol 26. Springer Science \& Business Media, Berlin

40. Kunin IA (2012) Elastic media with microstructure II: threedimensional models (Springer Series in Solid-State Sciences). Springer, softcover reprint of the original 1st ed. 1983 ed., 1

41. Silling SA, Epton M, Weckner O, Xu J, Askari E (2007) Peridynamic states and constitutive modeling. J Elast 88(2):151-184

42. Zhang Y, Qiao P (2019) A new bond failure criterion for ordinary state-based peridynamic mode ii fracture analysis. Int J Fract 215(1-2):105-128

43. Dipasquale D, Shojaei A, Yooyen S (2020) A novel stress tensorbased failure criterion for peridynamics. In: Multidisciplinary Digital Publishing Institute Proceedings, 39:23

44. Foster JT, Silling SA, Chen W (2011) An energy based failure criterion for use with peridynamic states. International Journal for Multiscale Computational Engineering, 9(6)

45. Willberg C, Wiedemann L, Rädel M (2019) A mode-dependent energy-based damage model for peridynamics and its implementation. J Mech Mater Struct 14(2):193-217 
46. Lipton R (2014) Dynamic brittle fracture as a small horizon limit of peridynamics. J Elast 117:21-50

47. Lipton R (2016) Cohesive dynamics and brittle fracture. J Elast 124(2):143-191

48. Mumford D, Shah J (1989) Optimal approximations by piecewise smooth functions and associated variational problem. Commun Pure Appl Math 42:577-685

49. Gobbino M (1998) Finite difference approximation of the mumford-shah functional. Commun Pure Appl Math 51:197-228

50. Ganzenmueller G, Hiermaier S, May M (2013) Improvements to the prototype micro-brittle linear elasticity model of peridynamics. Lect Notes Comput Sci Eng 100:12

51. Hu W, Ha YD, Bobaru F (2011) Modeling dynamic fracture and damage in a fiber-reinforced composite lamina with peridynamics. International Journal for Multiscale Computational Engineering, 9(6)

52. Hu W, Ha YD, Bobaru F (2012) Peridynamic model for dynamic fracture in unidirectional fiber-reinforced composites. Comput Methods Appl Mech Eng 217-220:247-261

53. Kilic B, Madenci E (2009) Prediction of crack paths in a quenched glass plate by using peridynamic theory. Int J Fract 156:165-177

54. Kilic B (2008) Peridynamic Theory for Progressive Failure Prediction in Homogeneous and Heterogeneous Materials. The University of Arizona

55. Diehl P, Lipton R, Schweitzer M (2016) Numerical verification of a bond-based softening peridynamic model for small displacements: deducing material parameters from classical linear theory. Institut für Numerische Simulation Preprint, 1630

56. Gerstle W, Sakhavand N, Chapman S (2010) Peridynamic and continuum models of reinforced concrete lap splice compared. Fracture Mechanics of Concrete and Concrete Structures-Recent Advances in Fracture Mechanics of Concrete

57. Aziz A (2014) Simulation of fracture of concrete using micropolar peridynamics. Ph.D thesis, The University of New Mexico

58. Silling SA (2019) Attenuation of waves in a viscoelastic peridynamic medium. Math Mech Solids 24(11):3597-3613

59. Hu Y, Madenci E (2017) Peridynamics for fatigue life and residual strength prediction of composite laminates. Compos Struct 160:169-184

60. Askari E, Xu J, Silling S (2006) Peridynamic analysis of damage and failure in composites. In: 44th AIAA aerospace sciences meeting and exhibit, $\mathrm{p} 88$

61. Hu Y, De Carvalho N, Madenci E (2015) Peridynamic modeling of delamination growth in composite laminates. Compos Struct 132:610-620

62. Mehrmashhadi J, Chen Z, Zhao J, Bobaru F (2019) A stochastically homogenized peridynamic model for intraply fracture in fiber-reinforced composites. Compos Sci Technol 182:107770

63. Zhang T, Zhou X (2019) A modified axisymmetric ordinary state-based peridynamics with shear deformation for elastic and fracture problems in brittle solids. Eur J Mech A Solids 77:103810

64. Lai X, Liu L, Zeleke M, Liu Q, Wang Z (2018) A non-ordinary state-based peridynamics modeling of fractures in quasi-brittle materials. Int J Impact Eng 111:01

65. Gao Y, Oterkus S (2019) Ordinary state-based peridynamic modelling for fully coupled thermoelastic problems. Continuum Mech Thermodyn 31(4):907-937

66. Rahaman MM, Roy P, Roy D, Reddy J (2017) A peridynamic model for plasticity: Micro-inertia based flow rule, entropy equivalence and localization residuals," Computer Methods in Applied Mechanics and Engineering, vol. 327, pp. 369-391, Advances in Computational Mechanics and Scientific Computation-the Cutting Edge

67. Kružík M, Mora-Corral C, Stefanelli U (2018) Quasistatic elastoplasticity via peridynamics: existence and localization. Continuum Mech Thermodyn 30:1155-1184
68. Madenci E, Oterkus S (2016) Ordinary state-based peridynamics for plastic deformation according to von mises yield criteria with isotropic hardening. J Mech Phys Solids 86:192-219

69. Kazemi SR (2020) Plastic deformation due to high-velocity impact using ordinary state-based peridynamic theory. Int $\mathrm{J}$ Impact Eng 137:103470

70. Oterkus E (2010) Peridynamic theory for modeling threedimensional damage growth in metallic and composite structures. $\mathrm{Ph} . \mathrm{D}$ thesis, The University of Arizona

71. Silling SA, Parks ML, Kamm JR, Weckner O, Rassaian M (2017) Modeling shockwaves and impact phenomena with eulerian peridynamics. Int J Impact Eng 107:47-57

72. Mitchell J, Silling S, Littlewood D (2015) A position-aware linear solid constitutive model for peridynamics. J Mech Mater Struct 10(5):539-557

73. Weckner O, Mohamed NAN (2013) Viscoelastic material models in peridynamics. Appl Math Comput 219(11):6039-6043

74. Mitchell JA (2011) A non-local, ordinary-statebasedviscoelasticity model forperidynamics. SANDIA REPORT, vol. SAND2011-806

75. Delorme R, Tabiai I, Laberge Lebel L, Lévesque M (2017) Generalization of the ordinary state-based peridynamic model for isotropic linear viscoelasticity. Mech Time-Depend Mater 21:549-575

76. Madenci E, Oterkus S (2017) Ordinary state-based peridynamics for thermoviscoelastic deformation. Eng Fract Mech 175:31-45

77. Tupek M, Radovitzky R (2014) An extended constitutive correspondence formulation of peridynamics based on nonlinear bond-strain measures. J Mech Phys Solids 65:82-92

78. O'Grady J, Foster J (2014) Peridynamic plates and flat shells: A non-ordinary, state-based model. Int J Solids Struct 51(25):45724579

79. Yaghoobi A, Chorzepa M, Kim S et al (2017) Mesoscale fracture analysis of multiphase cementitious composites using peridynamics. Materials 10(2):162

80. Chen X, Gunzburger M (2011) Continuous and discontinuous finite element methods for a peridynamics model of mechanics. Comput Methods Appl Mech Eng 200(9-12):1237-1250

81. Jha PK, Lipton R (2020) Finite element convergence for statebased peridynamic fracture models. Commun Appl Math Comput 2:93-128

82. Macek RW, Silling SA (2007) Peridynamics via finite element analysis. Finite Elem Anal Des 43(15):1169-1178

83. Weckner O, Emmrich E (2005) Numerical simulation of the dynamics of a nonlocal, inhomogeneous, infinite bar. J Comput Appl Mech 6(2):311-319

84. Parks ML, Lehoucq RB, Plimpton SJ, Silling SA (2008) Implementing peridynamics within a molecular dynamics code. Comput Phys Commun 179(11):777-783

85. Emmrich E, Weckner O (2007) The peridynamic equation and its spatial discretisation. Math Model Anal 12(1):17-27

86. Littlewood DJ (2015) Roadmap for peridynamic software implementation. Tech. Rep. 2015-9013, Sandia National Laboratories

87. Parks M, Littlewood D, Mitchell J, Silling S (2012) Peridigm users' guide. Tech. Rep. SAND2012-7800, Sandia National Laboratories

88. Diehl P, Jha PK, Kaiser H, Lipton R, Lévesque M (2020) An asynchronous and task-based implementation of peridynamics utilizing hpx-the c++ standard library for parallelism and concurrency. SN Appl Sci 2:2144

89. Jha PK, Diehl P (2021) Nlmech: Implementation of finite difference/meshfree discretization of nonlocal fracture models. Journal of Open Source Software 6(65):3020

90. Heller T, Diehl P, Byerly Z, Biddiscombe J, Kaiser H (2017) "Hpx-an open source c++ standard library for parallelism and concurrency," Proceedings of OpenSuCo, p. 5 
91. Kaiser H, Diehl P, Lemoine AS, Lelbach BA, Amini P, Berge A, Biddiscombe J, Brandt SR, Gupta N, Heller T, Huck K, Khatami Z, Kheirkhahan A, Reverdell A, Shirzad S, Simberg M, Wagle B, Wei W, Zhang T (2020) Hpx - the c++ standard library for parallelism and concurrency. J Open Sour Softw 5(53):2352

92. Boys B, Dodwell TJ, Hobbs M, Girolami M (2021) Peripy-a high performance opencl peridynamics package. arXiv preprint arXiv:2105.04150

93. Mossaiby F, Shojaei A, Zaccariotto M, Galvanetto U (2017) Opencl implementation of a high performance $3 \mathrm{~d}$ peridynamic model on graphics accelerators. Comput Math Appl 74(8):18561870

94. Diehl P (2012) Implementierung eines Peridynamik-Verfahrens auf GPU. diplomarbeit. University of Stuttgart, Institute of Parallel and Distributed Systems

95. Diehl P, Schweitzer MA (2015) Efficient neighbor search for particle methods on gpus. Meshfree methods for partial differential equations VII. Springer, Berlin, pp 81-95

96. Ren B, Wu C, Askari E (2017) A 3d discontinuous galerkin finite element method with the bond-based peridynamics model for dynamic brittle failure analysis. Int J Impact Eng 99:14-25

97. Silling S (2001) Peridynamic modeling of the kalthoff-winkler experiment, Submission for the

98. Emmrich E, Weckner O et al (2007) On the well-posedness of the linear peridynamic model and its convergence towards the navier equation of linear elasticity. Commun Math Sci 5(4):851-864

99. Du Q, Zhou K (2011) Mathematical analysis for the peridynamic nonlocal continuum theory. ESAIM: Math Model Numer Anal 45(2):217-234

100. Erbay HA, Erkip A, Muslu GM (2012) The cauchy problem for a one-dimensional nonlinear elastic peridynamic model. J Differ Equ 252(8):4392-4409

101. Du Q, Kamm JR, Lehoucq RB, Parks ML (2012) A new approach for a nonlocal, nonlinear conservation law. SIAM J Appl Math 72(1):464-487

102. Emmrich E, Puhst D (2013) Well-posedness of the peridynamic model with lipschitz continuous pairwise force function. Commun Math Sci 11(4):1039-1049

103. Bellido JC, Mora-Corral C (2014) Existence for nonlocal variational problems in peridynamics. SIAM J Math Anal 46(1):890916

104. Mengesha T, Du Q (2015) On the variational limit of a class of nonlocal functionals related to peridynamics. Nonlinearity 28(11):3999

105. Aksoylu B, Parks ML (2011) Variational theory and domain decomposition for nonlocal problems. Appl Math Comput 217(14):6498-6515

106. Du Q, Gunzburger M, Lehoucq RB, Zhou K (2012) Analysis and approximation of nonlocal diffusion problems with volume constraints. SIAM Rev 54(4):667-696

107. Du Q, Gunzburger M, Lehoucq R, Zhou K (2013) Analysis of the volume-constrained peridynamic navier equation of linear elasticity. J Elast 113(2):193-217

108. Du Q, Gunzburger M, Lehoucq RB, Zhou K (2013) A nonlocal vector calculus, nonlocal volume-constrained problems, and nonlocal balance laws. Math Models Methods Appl Sci 23(03):493-540

109. Seleson P, Du Q, Parks ML (2016) On the consistency between nearest-neighbor peridynamic discretizations and discretized classical elasticity models. Comput Methods Appl Mech Eng 311:698-722

110. Ganzenmüller GC, Hiermaier S, May M (2015) On the similarity of meshless discretizations of peridynamics and smooth-particle hydrodynamics. Computers \& Structures 150:71-78
111. Du Q, Tian X (2015) Robust discretization of nonlocal models related to peridynamics. Meshfree methods for partial differential equations VII. Springer, Berlin, pp 97-113

112. Zhou K, Du Q (2010) Mathematical and numerical analysis of linear peridynamic models with nonlocal boundary conditions. SIAM J Numer Anal 48(5):1759-1780

113. Tian X, Du Q (2013) Analysis and comparison of different approximations to nonlocal diffusion and linear peridynamic equations. SIAM J Numer Anal 51(6):3458-3482

114. Tian X, Du Q (2014) Asymptotically compatible schemes and applications to robust discretization of nonlocal models. SIAM J Numer Anal 52(4):1641-1665

115. Diehl P, Franzelin F, Pflüger D, Ganzenmüller GC (2016) Bondbased peridynamics: a quantitative study of mode i crack opening. Int J Fract 201(2): 157-170

116. Franzelin F, Diehl P, Pflüger D (2015) Non-intrusive uncertainty quantification with sparse grids for multivariate peridynamic simulations. Meshfree methods for partial differential equations VII. Springer, Berlin, pp 115-143

117. Jha PK, Lipton R (2018) Well-posed nonlinear nonlocal fracture models associated with double-well potentials, pp. 1-40. Springer International Publishing

118. Emmrich E, Puhst D (2016) A short note on modelling damage in peridynamics. J Elast 123:245-252

119. Du Q, Tao Y, Tian X (2017) A peridynamic model of fracture mechanics with bond-breaking. J Elast 132:197-218

120. Lipton R, Said E, Jha P (2018) Free damage propagation with memory. J Elast 133(2):129-153

121. Jha PK, Lipton R (2018) Numerical analysis of nonlocal fracture models in holder space. SIAM J Numer Anal 56(2):906-941

122. Jha PK, Lipton R (2019) Numerical convergence of finite difference approximations for state based peridynamic fracture models. Comput Methods Appl Mech Eng 351:184-225

123. Jha PK, Lipton R (2021) Finite element approximation of nonlocal dynamic fracture models. Discret Continuous Dyn Syst - B 26(3):1675-1710

124. Lipton RP, Lehoucq RB, Jha PK (2019) Complex fracture nucleation and evolution with nonlocal elastodynamics. J Peridyn Nonlocal Model 1(2):122-130

125. Lipton RP, Jha PK (2021) Nonlocal elastodynamics and fracture. Nonlinear Differential Equations and Applications

126. DalMaso G, Toader R (2019) On the cauchy problem for the wave equation on time-dependent domains. J Differ Equ 266(6):32093246

127. Trask N, You H, Yu Y, Parks ML (2007) An asymptotically compatible meshfree quadrature rule for nonlocal problems with applications to peridynamics. Comput Methods Appl Mech Eng 343:151-165

128. Jha PK, Lipton R (2020) Kinetic relations and local energy balance for LEFM from a nonlocal peridynamic model. Int J Fract 226(1):81-95

129. Diehl P (2017) Modeling and simulation of cracks and fractures with peridynamics in brittle materials. Ph.D thesis, University of Bonn, Germany

130. Seleson P, Parks ML, Gunzburger M, Lehoucq RB (2009) Peridynamics as an upscaling of molecular dynamics. Multiscale Model Simul 8(1):204-227

131. Bessa M, Foster J, Belytschko T, Liu WK (2014) A meshfree unification: reproducing kernel peridynamics. Comput Mech 53(6): $1251-1264$

132. Bode T, Weißenfels C, Wriggers P (2020) Peridynamic petrovgalerkin method: a generalization of the peridynamic theory of correspondence materials. Comput Methods Appl Mech Eng 358:112636

133. Hillman M, Pasetto M, Zhou G (2020) Generalized reproducing kernel peridynamics: unification of local and non-local meshfree 
methods, non-local derivative operations, and an arbitrary-order state-based peridynamic formulation. Comput Particle Mech 7(2):435-469

134. Madenci E, Barut A, Futch M (2016) Peridynamic differential operator and its applications. Comput Methods Appl Mech Eng 304:408-451

135. Shojaei A, Galvanetto U, Rabczuk T, Jenabi A, Zaccariotto M (2019) A generalized finite difference method based on the peridynamic differential operator for the solution of problems in bounded and unbounded domains. Comput Methods Appl Mech Eng 343:100-126

136. Ahrens J, Geveci B, Law C (2005) Paraview: An end-user tool for large data visualization. The visualization handbook, 717

137. Childs H, Brugger E, Whitlock B, Meredith J, Ahern S, Pugmire D, Biagas K, Miller M, Harrison C, Weber GH, Krishnan H, Fogal T, Sanderson A, Garth C, Bethel EW, Camp D, Rübel O, Durant M, Favre JM, Navrátil P (Oct 2012) VisIt: An end-user tool for visualizing and analyzing very large data. In: High Performance Visualization-Enabling Extreme-Scale Scientific Insight, pp. 357-372, Open Access Publications from the University of California

138. Levine JA, Bargteil AW, Corsi C, Tessendorf J, Geist R (2014) A peridynamic perspective on spring-mass fracture. In: Proceedings of the ACM SIGGRAPH/Eurographics Symposium on Computer Animation, pp. 47-55, Eurographics Association

139. Chen W, Zhu F, Zhao J, Li S, Wang G (2018) Peridynamics-based fracture animation for elastoplastic solids. In: Computer Graphics Forum, 37:112-124, Wiley Online Library

140. Xu L, He X, Chen W, Li S, Wang G (2018) Reformulating hyperelastic materials with peridynamic modeling. In: Computer Graphics Forum, 37:121-130, Wiley Online Library

141. Diehl P, Bußler M, Pflüger D, Frey S, Ertl T, Sadlo F, Schweitzer MA (2017) Extraction of fragments and waves after impact damage in particle-based simulations. In: Meshfree Methods for Partial Differential Equations VIII, pp. 17-34, Springer

142. Littlewood D, Silling S, Demmie P (2016) Identification of fragments in a meshfree peridynamic simulation. In: ASME 2016 International Mechanical Engineering Congress and Exposition, pp. V009T12A071-V009T12A071, American Society of Mechanical Engineers

143. Bussler M, Diehl P, Pflüger D, Frey S, Sadlo F, Ertl T, Schweitzer MA (2017) Visualization of fracture progression in peridynamics. Comput Gr 67:45-57

144. Francfort G, Marigo J-J (1998) Revisiting brittle fracture as an energy minimization problem. J Mech Phys Solids 46(8):13191342

145. Aranson I, Kalatsky V, Vinokur V (2000) Continuum field description of crack propagation. Phys Rev Lett 85(1):118

146. Bourdin B, Francfort GA, Marigo J-J (2000) Numerical experiments in revisited brittle fracture. J Mech Phys Solids 48(4):797826

147. Francfort GA, Larsen CJ (2003) Existence and convergence for quasi-static evolution in brittle fracture. Commun Pure Appl Math 56(10): 1465-1500

148. dal Maso G, Francfort GA, Toader R (2005) Quasistatic crack growth in nonlinear elasticity. Arch Ration Mech Anal 176:165225

149. Ambrosio L, Tortorelli V (1990) Approximation of functionals depending on jumps by elliptic functionals via $\gamma$-convergence. Commun Pure Appl Math 43:999-1036

150. Ambrosio L, Tortorelli V (1992) On the approximation of free discontinuity problems. Boll Un Mat Ital B 6:105-123

151. Miehe C, Welschinger F, Hofacker M (2010) Thermodynamically consistent phase-field models of fracture: Variational principles and multi-field fe implementations. Int J Numer Meth Eng 83(10):1273-1311
152. Bourdin B, Marigo J-J, Maurini C, Sicsic P (2014) Morphogenesis and propagation of complex cracks induced by thermal shocks. Phys Rev Lett 112(1):014301

153. Wu J-Y (2017) A unified phase-field theory for the mechanics of damage and quasi-brittle failure. J Mech Phys Solids 103:72-99

154. Kuhn C, Müller R (2008) A phase field model for fracture. PAMM 8(1):10223-10224

155. Kuhn C, Müller R (2010) A continuum phase field model for fracture. Eng Fract Mech 77(18):3625-3634

156. Pham K, Marigo J-J (2010) Approche variationnelle de l'endommagement?: I. les concepts fondamentaux. Comptes Rendus Mécanique 338(4):191-198

157. Pham K, Marigo J-J (2010) Approche variationnelle de l'endommagement?: Ii. les modèles à gradient. Comptes Rendus Mécanique 338(4):199-206

158. Borden MJ, Verhoosel CV, Scott MA, Hughes TJR, Landis CM (2012) A phase-field description of dynamic brittle fracture. Comput Meth Appl Mech Engrg 217:77-95

159. Sargado JM, Keilegavlen E, Berre I, Nordbotten JM (2018) Highaccuracy phase-field models for brittle fracture based on a new family of degradation functions. J Mech Phys Solids 111:458489

160. Arriaga M, Waisman $H$ (2018) Multidimensional stability analysis of the phase-field method for fracture with a general degradation function and energy split. Comput Mech 61:181-205

161. Braides A (1998) Approximation of free-discontinuity problems. Springer Science \& Business Media, Berlin

162. Pham K, Ravi-Chandar K, Landis C (2017) Experimental validation of a phase-field model for fracture. Int J Fract 205(1):83-101

163. Egger A, Pillai U, Agathos K, Kakouris E, Chatzi E, Aschroft IA, Triantafyllou SP (2019) Discrete and phase field methods for linear elastic fracture mechanics: a comparative study and stateof-the-art review. Appl Sci 9(12):2436

164. Neitzel I, Wick T, Wollner W (2017) An optimal control problem governed by a regularized phase-field fracture propagation model. SIAM J Control Optim 55(4):2271-2288

165. Mikelić A, Wheeler MF, Wick T (2015) A quasi-static phase-field approach to pressurized fractures. Nonlinearity 28(5):1371-1399

166. Osher S, Sethian J (1988) Fronts propagating with curvaturedependent speed: algorithms based on Hamiltonian-Jacobi formulations. J Comput Phys 79(1):12-49

167. Lee S, Wheeler MF, Wick T (2017) Iterative coupling of flow, geomechanics and adaptive phase-field fracture including levelset crack width approaches. J Comput Appl Math 314:40-60

168. Wheeler MF, Wick T, Lee S (2020) IPACS: Integrated Phase-Field Advanced Crack Propagation Simulator. An adaptive, parallel, physics-based-discretization phase-field framework for fracture propagation in porous media. Comput Methods Appl Mech Eng 367:113124

169. dal Maso G, Toader R (2002) A model for the quasistatic growth of brittle fractures: existence and approximation results. Arch Ration Mech Anal 162:101-135

170. Hill R (1958) A general theory of uniqueness and stability in elastic-plastic solids. J Mech Phys Solids 6(3):236-249

171. Gerasimov T, Römer U, Vondřejc J, Matthies HG, De Lorenzis L (2020) Stochastic phase-field modeling of brittle fracture: Computing multiple crack patterns and their probabilities. Comput Methods Appl Mech Eng 372:113353

172. Larsen CJ, Ortner C, Süli E (2010) Existence of solutions to a regularized model of dynamic fracture. Math Models Methods Appl Sci 20(07):1021-1048

173. Chambolle A, Giacomini A, Ponsiglione M (2008) Crack initiation in brittle materials. Arch Ration Mech Anal 188:309-349

174. van Goethem N, Novotny A (2010) Crack nucleation sensitivity analysis. Math Methods Appl Sci, 33(16) 
175. Kumar A, Bourdin B, Francfort G, Lopez-Pamies O (2020) Revisiting nucleation in the phase-field approach to brittle fracture. $\mathrm{J}$ Mech Phys Solids 142:104027

176. de Lorenzis L, Maurini C (2021) Nucleation under multi-axial loading in variational phase-field models of brittle fracture. Int $\mathbf{J}$ Fract. https://doi.org/10.1007/s10704-021-00555-6

177. Chambolle A, Francfort G, Marigo J-J (2009) When and how do cracks propagate? J Mech Phys Solids 57(9):1614-1622

178. Mielke A (2005) Evolution of rate-independent systems. Elsevier, North-Holland, pp 461-559

179. Pham K, Amor H, Marigo J-J, Maurini C (2011) Gradient damage models and their use to approximate brittle fracture. Int J Damage Mech 20(4):618-652

180. Pham K, Marigo J-J, Maurini C (2011) The issues of the uniqueness and the stability of the homogeneous response in uniaxial tests with gradient damage models. J Mech Phys Solids 59(6): 1163-1190

181. Pham K, Marigo J (2013) From the onset of damage to rupture: construction of responses with damage localization for a general class of gradient damage models. Continuum Mech Thermodyn 25:147-171

182. Nguyen Q (1987) Bifurcation and postbifurcation analysis in plasticity and brittle fracture. J Mech Phys Solids 35:303-324

183. Nguyen Q (2000) Stability and nonlinear solid mechanics. Wiley, London

184. Benallal A, Marigo J-J (2006) Bifurcation and stability issues in gradient theories with softening. Modell Simul Mater Sci Eng 15:S283-S295

185. de Borst R, Verhoosel CV (2016) Gradient damage vs phase-field approaches for fracture: Similarities and differences. Computer Methods in Applied Mechanics and Engineering, 312:78-94, Phase Field Approaches to Fracture

186. Miehe C, Hofacker M, Welschinger F (2010) A phase field model for rate-independent crack propagation: Robust algorithmic implementation based on operator splits. Comput Methods Appl Mech Eng 199(45-48):2765-2778

187. Burke S, Ortner C, Süli E (2010) An adaptive finite element approximation of a variational model of brittle fracture. SIAM J Numer Anal 48(3):980-1012

188. Salman O, Truskinovsky L (2021) De-localizing brittle fracture. J Mech Phys Solids 154:104517

189. Caputo M, Fabrizio M (2015) Damage and fatigue described by a fractional derivative model. J Comput Phys 293:400-408

190. Amendola G, Fabrizio M, Golden J (2016) Thermomechanics of damage and fatigue by a phase field model. J Therm Stresses 39(5):487-499

191. Boldrini J, de Moraes EB, Chiarelli L, Fumes F, Bittencourt M (2016) A non-isothermal thermodynamically consistent phase field framework for structural damage and fatigue. Comput Methods Appl Mech Eng 312:395-427

192. Alessi R, Vidoli S, De Lorenzis L (2018) A phenomenological approach to fatigue with a variational phase-field model: The onedimensional case. Eng Fract Mech 190:53-73

193. Seiler M, Hantschke P, Brosius A, Kästner M (2018) A numerically efficient phase-field model for fatigue fracture-1d analysis. PAMM 18(1):e201800207

194. Mesgarnejad A, Imanian A, Karma A (2019) Phase-field models for fatigue crack growth. Theoret Appl Fract Mech 103:102282

195. Seleš K, Aldakheel F, Tonković Z, Sorić J, Wriggers P (2021) A general phase-field model for fatigue failure in brittle and ductile solids. Comput Mech 67:1431-1452

196. Carrara P, Ambati M, Alessi R, De Lorenzis L (2020) A framework to model the fatigue behavior of brittle materials based on a variational phase-field approach. Comput Methods Appl Mech Eng 361:112731
197. Miehe C, Schaenzel L-M, Ulmer H (2015) Phase field modeling of fracture in multi-physics problems. part i. balance of crack surface and failure criteria for brittle crack propagation in thermo-elastic solids. Comput Methods Appl Mech Eng 294:449-485

198. Schlüter A, Kuhn C, Müller R (2017) Simulation of laser-induced controlled fracturing utilizing a phase field model. J Comput Inf Sci Eng 17(2):021001

199. Miehe C, Hofacker M, Schänzel L-M, Aldakheel F (2015) Phase field modeling of fracture in multi-physics problems. part ii. coupled brittle-to-ductile failure criteria and crack propagation in thermo-elastic-plastic solids. Comput Methods Appl Mech Eng 294:486-522

200. Radszuweit M, Kraus C (2017) Modeling and simulation of non-isothermal rate-dependent damage processes in inhomogeneous materials using the phase-field approach. Comput Mech 60(1):163-179

201. Mikelic A, Wheeler MF, Wick T (2015) A phase-field method for propagating fluid-filled fractures coupled to a surrounding porous medium. Multiscale Model Simul 13(1):367-398

202. Lee S, Wheeler MF, Wick T (2016) Pressure and fluid-driven fracture propagation in porous media using an adaptive finite element phase field model. Comput Methods Appl Mech Eng 305:111132

203. Zhou S, Zhuang X, Rabczuk T (2018) A phase-field modeling approach of fracture propagation in poroelastic media. Eng Geol 240:189-203

204. Shanthraj P, Svendsen B, Sharma L, Roters F, Raabe D (2017) Elasto-viscoplastic phase field modelling of anisotropic cleavage fracture. J Mech Phys Solids 99:19-34

205. Diehl M, Wicke M, Shanthraj P, Roters F, Brueckner-Foit A, Raabe D (2017) Coupled crystal plasticity-phase field fracture simulation study on damage evolution around a void: pore shape versus crystallographic orientation. JOM 69(5):872-878

206. Duda FP, Ciarbonetti A, Toro S, Huespe AE (2018) A phase-field model for solute-assisted brittle fracture in elastic-plastic solids. Int J Plast 102:16-40

207. Nguyen T-T, Bolivar J, Réthoré J, Baietto M-C, Fregonese M (2017) A phase field method for modeling stress corrosion crack propagation in a nickel base alloy. Int J Solids Struct 112:65-82

208. Martínez-Pañeda E, Golahmar A, Niordson CF (2018) A phase field formulation for hydrogen assisted cracking. Comput Methods Appl Mech Eng 342:742-761

209. Wu T, De Lorenzis L (2016) A phase-field approach to fracture coupled with diffusion. Comput Methods Appl Mech Eng 312:196-223

210. Klinsmann M, Rosato D, Kamlah M, McMeeking RM (2016) Modeling crack growth during li insertion in storage particles using a fracture phase field approach. J Mech Phys Solids 92:313344

211. Roubicek T, Vodicka R (2019) A monolithic model for phase-field fracture and waves in solid-fluid media towards earthquakes. Int J Fract 219:135-152

212. Kruzik M, Roubicek T (2019) Mathematical methods in continuum mechanics of solids. Springer, Berlin

213. Fei F, Choo J (2019) A phase-field method for modeling cracks with frictional contact. Int J Numer Meth Eng 121:740-762

214. Ulmer H, Hofacker M, Miehe C (2012) Phase field modeling of fracture in plates and shells. PAMM 12(1):171-172

215. Mesgarnejad A, Bourdin B, Khonsari M (2013) A variational approach to the fracture of brittle thin films subject to out-of-plane loading. J Mech Phys Solids 61(11):2360-2379

216. Amiri F, Millán D, Shen Y, Rabczuk T, Arroyo M (2014) Phasefield modeling of fracture in linear thin shells. Theoret Appl Fract Mech 69:102-109 
217. Ambati M, De Lorenzis L (2016) Phase-field modeling of brittle and ductile fracture in shells with isogeometric nurbs-based solidshell elements. Comput Methods Appl Mech Eng 312:351-373

218. Areias P, Rabczuk T, Msekh M (2016) Phase-field analysis of finite-strain plates and shells including element subdivision. Comput Methods Appl Mech Eng 312:322-350

219. Reinoso J, Paggi M, Linder C (2017) Phase field modeling of brittle fracture for enhanced assumed strain shells at large deformations: formulation and finite element implementation. Comput Mech 59(6):981-1001

220. Kiendl J, Ambati M, De Lorenzis L, Gomez H, Reali A (2016) Phase-field description of brittle fracture in plates and shells. Computer Methods in Applied Mechanics and Engineering, vol. 312, pp. 374-394. Phase Field Approaches to Fracture

221. Hofacker M, Miehe C (2012) Continuum phase field modeling of dynamic fracture: variational principles and staggered fe implementation. Int J Fract 178(1-2):113-129

222. Borden MJ, Verhoosel CV, Scott MA, Hughes TJ, Landis CM (2012) A phase-field description of dynamic brittle fracture. Comput Methods Appl Mech Eng 217:77-95

223. Borden MJ, Hughes TJ, Landis CM, Verhoosel CV (2014) A higher-order phase-field model for brittle fracture: Formulation and analysis within the isogeometric analysis framework. Comput Methods Appl Mech Eng 273:100-118

224. Mesgarnejad A, Bourdin B, Khonsari M (2015) Validation simulations for the variational approach to fracture. Comput Methods Appl Mech Eng 290:420-437

225. Borden MJ, Hughes TJ, Landis CM, Anvari A, Lee IJ (2016) A phase-field formulation for fracture in ductile materials: Finite deformation balance law derivation, plastic degradation, and stress triaxiality effects. Comput Methods Appl Mech Eng 312:130-166

226. Liu G, Li Q, Msekh MA, Zuo Z (2016) Abaqus implementation of monolithic and staggered schemes for quasi-static and dynamic fracture phase-field model. Comput Mater Sci 121:35-47

227. Gültekin O, Dal H, Holzapfel GA (2016) A phase-field approach to model fracture of arterial walls: theory and finite element analysis. Comput Methods Appl Mech Eng 312:542-566

228. Pham K, Ravi-Chandar K (2017) The formation and growth of echelon cracks in brittle materials. Int J Fract 206(2):229-244

229. Miehe C, Schänzel L-M (2014) Phase field modeling of fracture in rubbery polymers. part i: Finite elasticity coupled with brittle failure. J Mech Phys Solids 65:93-113

230. Hesch C, Weinberg K (2014) Thermodynamically consistent algorithms for a finite-deformation phase-field approach to fracture. Int J Numer Meth Eng 99(12):906-924

231. Raina A, Miehe C (2016) A phase-field model for fracture in biological tissues. Biomech Model Mechanobiol 15(3):479-496

232. Hesch C, Gil A, Ortigosa R, Dittmann M, Bilgen C, Betsch P, Franke M, Janz A, Weinberg K (2017) A framework for polyconvex large strain phase-field methods to fracture. Comput Methods Appl Mech Eng 317:649-683

233. Lee S, Yoon HC, Muddamallappa MS (2020) Nonlinear strainlimiting elasticity for fracture propagation with phase-field approach

234. Wu J, McAuliffe C, Waisman H, Deodatis G (2016) Stochastic analysis of polymer composites rupture at large deformations modeled by a phase field method. Computer Methods in Applied Mechanics and Engineering, 312:596-634. Phase Field Approaches to Fracture

235. Bourdin B, Larsen CJ, Richardson CL (2011) A time-discrete model for dynamic fracture based on crack regularization. Int $\mathrm{J}$ Fract 168(2):133-143

236. Schlüter A, Willenbücher A, Kuhn C, Müller R (2014) Phase field approximation of dynamic brittle fracture. Comput Mech 54(5):1141-1161
237. Li T, Marigo J-J, Guilbaud D, Potapov S (2016) Gradient damage modeling of brittle fracture in an explicit dynamics context. Int $\mathrm{J}$ Numer Meth Eng 108(11):1381-1405

238. Roubicek T (2019) Models of dynamic damage and phase-field fracture, and their various time discretisations

239. Geelen RJ, Liu Y, Hu T, Tupek MR, Dolbow JE (2019) A phasefield formulation for dynamic cohesive fracture. Comput Methods Appl Mech Eng 348:680-711

240. Arriaga M, Waisman H (2017) Combined stability analysis of phase-field dynamic fracture and shear band localization. Int $\mathrm{J}$ Plast 96:81-119

241. Mandal TK, Nguyen VP, Wu J-Y (2020) Evaluation of variational phase-field models for dynamic brittle fracture. Eng Fract Mech 235:107169

242. Verhoosel CV, de Borst R (2013) A phase-field model for cohesive fracture. Int J Numer Meth Eng 96(1):43-62

243. May S, Vignollet J, De Borst R (2015) A numerical assessment of phase-field models for brittle and cohesive fracture: $\gamma$-convergence and stress oscillations. Eur J Mech-A/Solids 52:72-84

244. Nguyen TT, Yvonnet J, Zhu Q-Z, Bornert M, Chateau C (2016) A phase-field method for computational modeling of interfacial damage interacting with crack propagation in realistic microstructures obtained by microtomography. Comput Methods Appl Mech Eng 312:567-595

245. Vignollet J, May S, De Borst R, Verhoosel CV (2014) Phase-field models for brittle and cohesive fracture. Meccanica 49(11):25872601

246. Fei F, Choo J (2021) Double-phase-field formulation for mixedmode fracture in rocks. Comput Methods Appl Mech Eng 376:113655

247. Chen L, de Borst R (2021) Phase-field modelling of cohesive fracture. Eur J Mech A Solids 90:104343

248. Duda FP, Ciarbonetti A, Sánchez PJ, Huespe AE (2015) A phasefield/gradient damage model for brittle fracture in elastic-plastic solids. Int J Plast 65:269-296

249. Alessi R, Marigo J-J, Vidoli S (2015) Gradient damage models coupled with plasticity: variational formulation and main properties. Mech Mater 80:351-367

250. Ambati M, Kruse R, De Lorenzis L (2016) A phase-field model for ductile fracture at finite strains and its experimental verification. Comput Mech 57(1):149-167

251. Ambati M, Gerasimov T, De Lorenzis L (2015) Phase-field modeling of ductile fracture. Comput Mech 55(5):1017-1040

252. Kuhn C, Noll T, Müller R (2016) On phase field modeling of ductile fracture. GAMM-Mitteilungen 39(1):35-54

253. Noii N, Khodadadian A, Ulloa J, Aldakheel F, Wick T, Francois S, Wriggers P (2021) Bayesian inversion for unified ductile phasefield fracture. Computational Mechanics

254. Dittmann M, Aldakheel F, Schulte J, Schmidt F, Krüger M, Wriggers P, Hesch C (2020) Phase-field modeling of porous-ductile fracture in non-linear thermo-elasto-plastic solids. Comput Methods Appl Mech Eng 361:112730

255. Kienle D, Aldakheel F, Keip M-A (2019) A finite-strain phasefield approach to ductile failure of frictional materials. Int J Solids Struct 172-173:147-162

256. Aldakheel F, Hudobivnik B, Wriggers P (2019) Virtual element formulation for phase-field modeling of ductile fracture. Int J Multiscale Comput Eng 17(2):181-200

257. Arriaga M, Waisman $\mathrm{H}$ (2018) Stability analysis of the phase-field method for fracture with a general degradation function and plasticity induced crack generation. Mechanics of Materials, vol. 116, pp. 33-48. IUTAM Symposium on Dynamic Instabilities in Solids

258. Choo J, Sun W (2018) Coupled phase-field and plasticity modeling of geological materials: From brittle fracture to ductile flow. Comput Methods Appl Mech Eng 330:1-32 
259. Bleyer J, Alessi R (2018) Phase-field modeling of anisotropic brittle fracture including several damage mechanisms. Comput Methods Appl Mech Eng 336:213-236

260. Hakim V, Karma A (2005) Crack path prediction in anisotropic brittle materials. Phys Rev Lett 95(23):235501

261. Clayton JD, Knap J (2014) A geometrically nonlinear phase field theory of brittle fracture. Int J Fract 189(2):139-148

262. Li B, Peco C, Millán D, Arias I, Arroyo M (2015) Phase-field modeling and simulation of fracture in brittle materials with strongly anisotropic surface energy. Int J Numer Meth Eng 102(3-4):711727

263. Nguyen TT, Réthoré J, Baietto M-C (2017) Phase field modelling of anisotropic crack propagation. Eur J Mech-A/Solids 65:279288

264. Teichtmeister S, Kienle D, Aldakheel F, Keip M-A (2017) Phase field modeling of fracture in anisotropic brittle solids. Int J NonLinear Mech 97:1-21

265. Baldelli AL, Babadjian J-F, Bourdin B, Henao D, Maurini C (2014) A variational model for fracture and debonding of thin films under in-plane loadings. J Mech Phys Solids 70:320-348

266. Hansen-Dörr AC, de Borst R, Hennig P, Kästner M (2019) Phasefield modelling of interface failure in brittle materials. Comput Methods Appl Mech Eng 346:25-42

267. Patil R, Mishra B, Singh I, Bui T (2018) A new multiscale phase field method to simulate failure in composites. Adv Eng Softw 126:9-33

268. Quintanas-Corominas A, Reinoso J, Casoni E, Turon A, Mayugo J (2019) A phase field approach to simulate intralaminar and translaminar fracture in long fiber composite materials. Compos Struct 220:899-911

269. Song L, Meng S, Xu C, Fang G, Yang Q (2019) Finite elementbased phase-field simulation of interfacial damage in unidirectional composite under transverse tension. Modell Simul Mater Sci Eng 27(5):055011

270. Spatschek R, Pilipenko D, Müller-Gugenberger C, Brener EA (2006) Phase field modeling of fracture and composite materials. Phys Rev Lett 96:015502

271. Denli FA, Gültekin O, Holzapfel GA, Dal H (2020) A phasefield model for fracture of unidirectional fiber-reinforced polymer matrix composites. Computational Mechanics, pp 1-18

272. Russ J, Slesarenko V, Rudykh S, Waisman H (2020) Rupture of 3d-printed hyperelastic composites: Experiments and phase field fracture modeling. J Mech Phys Solids 140:103941

273. Amor H, Marigo J-J, Maurini C (2009) Regularized formulation of the variational brittle fracture with unilateral contact: Numerical experiments. J Mech Phys Solids 57(8):1209-1229

274. Zhang X, Sloan SW, Vignes C, Sheng D (2017) A modification of the phase-field model for mixed mode crack propagation in rocklike materials. Comput Methods Appl Mech Eng 322:123-136

275. Strobl M, Seelig T (2015) A novel treatment of crack boundary conditions in phase field models of fracture. Pamm 15(1):155-156

276. Steinke C, Kaliske M (2019) A phase-field crack model based on directional stress decomposition. Comput Mech 63(5):1019-1046

277. Bryant EC, Sun W (2018) A mixed-mode phase field fracture model in anisotropic rocks with consistent kinematics. Comput Methods Appl Mech Eng 342:561-584

278. Freddi F, Royer-Carfagni G (2010) Regularized variational theories of fracture: a unified approach. J Mech Phys Solids 58(8):1154-1174

279. Bilgen C, Homberger S, Weinberg K (2019) Phase-field fracture simulations of the brazilian splitting test. Int J Fract 220:85-98

280. Fan M, Jin Y, Wick T (2021) A quasi-monolithic phase-field description for mixed-mode fracture using predictor-corrector mesh adaptivity. Engineering with Computers. Accepted

281. Bleyer J, Roux-Langlois C, Molinari J-F (2017) Dynamic crack propagation with a variational phase-field model: limiting speed, crack branching and velocity-toughening mechanisms. Int J Fract 204(1):79-100

282. Weinberg K, Wieners C (2021) Dynamic phase-field fracture with a first-order discontinuous galerkin method for elastic waves

283. Mang K, Wick T, Wollner W (2020) A phase-field model for fractures in nearly incompressible solids. Comput Mech 65:6178

284. Basava S, Mang K, Walloth M, Wick T, Wollner W (2020) Adaptive and pressure-robust discretization of incompressible pressure-driven phase-field fracture. accepted, SPP 1748 final report

285. Mang K, Fehse A, Kröger NH, Wick T (2021) A mixed phase-field fracture model for crack propagation in punctured epdm strips. Theoret Appl Fract Mech 115:103076

286. Kuhn C, Müller R (2009) Phase field simulation of thermomechanical fracture. In: PAMM: Proceedings in Applied Mathematics and Mechanics, vol. 9, pp 191-192, Wiley Online Library

287. Wheeler M, Wick T, Wollner W (2014) An augmentedLagangrian method for the phase-field approach for pressurized fractures. Comp Meth Appl Mech Engrg 271:69-85

288. Gerasimov T, Lorenzis LD (2019) On penalization in variational phase-field models of brittle fracture. Comput Methods Appl Mech Eng 354:990-1026

289. Neitzel I, Wick T, Wollner W (2019) An optimal control problem governed by a regularized phase-field fracture propagation model. part ii: The regularization limit. SIAM J Control Optim 57(3): 1672-1690

290. Heister T, Wheeler MF, Wick T (2015) A primal-dual active set method and predictor-corrector mesh adaptivity for computing fracture propagation using a phase-field approach. Comput Methods Appl Mech Eng 290:466-495

291. Wambacq J, Ulloa J, Lombaert G, François S (2020) Interior-point methods for the phase-field approach to brittle and ductile fracture

292. Kuhn C, Müller R (2011) A new finite element technique for a phase field model of brittle fracture. J Theor Appl Mech 49:01

293. Engwer C, Schumacher L (2017) A phase field approach to pressurized fractures using discontinuous galerkin methods. Mathematics and Computers in Simulation, vol. 137, pp. 266-285

294. Zhou JX, Li ME (2006) Solving phase field equations using a meshless method. Commun Numer Methods Eng 22(11):11091115

295. Li W, Nguyen-Thanh N, Zhou K (2020) Phase-field modeling of brittle fracture in a $3 \mathrm{~d}$ polycrystalline material via an adaptive isogeometric-meshfree approach. Int J Numer Meth Eng 121(22):5042-5065

296. Bourdin B (2007) Numerical implementation of a variational formulation of quasi-static brittle fracture. Interfaces Free Bound 9:411-430

297. Farrell P, Maurini C (2017) Linear and nonlinear solvers for variational phase-field models of brittle fracture. Int J Numer Meth Eng 109(5):648-667

298. Wu J-Y (2018) A geometrically regularized gradient-damage model with energetic equivalence. Comput Methods Appl Mech Eng 328:612-637

299. Wu J-Y (2018) Robust numerical implementation of non-standard phase-field damage models for failure in solids. Comput Methods Appl Mech Eng 340:767-797

300. Brun MK, Wick T, Berre I, Nordbotten JM, Radu FA (2020) An iterative staggered scheme for phase field brittle fracture propagation with stabilizing parameters. Comput Methods Appl Mech Eng 361:112752

301. Storvik E, Both JW, Sargado JM, Nordbotten JM, Radu FA (2021) An accelerated staggered scheme for phase-field modeling of brittle fracture. Comput Methods Appl Mech Eng 381:113822. https://doi.org/10.1016/j.cma.2021.113822 
302. Jammoul M, Wheeler MF, Wick T (2020) A phase-field multirate scheme with stabilized iterative coupling for pressure driven fracture propagation in porous media. Comput Math Appl 91:176191. https://doi.org/10.1016/j.camwa.2020.11.009

303. Engwer C, Pop IS, Wick T (2021) Dynamic and Weighted Stabilizations of the L-scheme Applied to a Phase-Field Model for Fracture Propagation. In: Vermolen FJ, Vuik C (eds) Numerical Mathematics and Advanced Applications ENUMATH 2019. Lecture Notes in Computational Science and Engineering, vol 139. Springer, Cham. https://doi.org/10.1007/978-3-030-558741 117

304. Gerasimov T, De Lorenzis L (2016) A line search assisted monolithic approach for phase-field computing of brittle fracture. Comput Methods Appl Mech Eng 312:276-303

305. Wick T (2017) Modified Newton methods for solving fully monolithic phase-field quasi-static brittle fracture propagation. Comput Methods Appl Mech Eng 325:577-611

306. Wick T (2017) An error-oriented Newton/inexact augmented Lagrangian approach for fully monolithic phase-field fracture propagation. SIAM J Sci Comput 39(4):B589-B617

307. Jodlbauer D, Langer U, Wick T (2020) Matrix-free multigrid solvers for phase-field fracture problems. Comput Methods Appl Mech Eng 372:113431

308. Kopanicakova A, Krause R (2020) A recursive multilevel trust region method with application to fully monolithic phase-field models of brittle fracture. Comput Methods Appl Mech Eng 360:112720

309. May S, Vignollet J, de Borst R (2016) A new arc-length control method based on the rates of the internal and the dissipated energy. Eng Comput 33(1):100-115

310. Singh N, Verhoosel C, De Borst R, Van Brummelen E (2016) A fracture-controlled path-following technique for phase-field modeling of brittle fracture. Finite Elem Anal Des 113:14-29

311. Heister T, Wick T (2018) Parallel solution, adaptivity, computational convergence, and open-source code of $2 \mathrm{~d}$ and $3 \mathrm{~d}$ pressurized phase-field fracture problems. PAMM 18(1):e201800353

312. Jodlbauer D, Langer U, Wick T (2020) Parallel matrix-free higherorder finite element solvers for phase-field fracture problems. Math Comput Appl 25(3):40

313. Ma R, Sun W (2020) FFT-based solver for higherorder and multiphase-field fracture models applied to strongly anisotropic brittle materials. Comput Methods Appl Mech Eng. https://doi.org/10. 1016/j.cma.2019.112781

314. Nguyen TT, Yvonnet J, Zhu Q-Z, Bornert M, Chateau C (2015) A phase field method to simulate crack nucleation and propagation in strongly heterogeneous materials from direct imaging of their microstructure. Eng Fract Mech 139:18-39

315. Noii N, Aldakheel F, Wick T, Wriggers P (2020) An adaptive global-local approach for phase-field modeling of anisotropic brittle fracture. Comput Methods Appl Mech Eng 361:112744

316. Zavattieri PD (2006) Modeling of crack propagation in thinwalled structures using a cohesive model for shell elements. J Appl Mech 73(6):948-958

317. Kuhn C, Schlüter A, Müller R (2015) On degradation functions in phase field fracture models. Comput Mater Sci 108:374-384

318. Steinke C, Özenç K, Chinaryan G, Kaliske M (2016) A comparative study of the r-adaptive material force approach and the phase-field method in dynamic fracture. Int J Fract 201(1):97-118

319. Msekh MA, Sargado JM, Jamshidian M, Areias PM, Rabczuk T (2015) Abaqus implementation of phase-field model for brittle fracture. Comput Mater Sci 96:472-484

320. Molnár G, Gravouil A (2017) 2d and 3d abaqus implementation of a robust staggered phase-field solution for modeling brittle fracture. Finite Elem Anal Des 130:27-38

321. Pillai U, Heider Y, Markert B (2018) A diffusive dynamic brittle fracture model for heterogeneous solids and porous materials with implementation using a user-element subroutine. Comput Mater Sci 153:36-47

322. Bhowmick S, Liu GR (2018) A phase-field modeling for brittle fracture and crack propagation based on the cell-based smoothed finite element method. Eng Fract Mech 204:369-387

323. Jeong H, Signetti S, Han T-S, Ryu S (2018) Phase field modeling of crack propagation under combined shear and tensile loading with hybrid formulation. Comput Mater Sci 155:483-492

324. Wu J-Y, Huang Y (2020) Comprehensive implementations of phase-field damage models in abaqus. Theoret Appl Fract Mech 106:102440

325. van Zwieten G, van Zwieten J, Verhoosel C, Fonn E, van Opstal T, Hoitinga W (2019) Nutils

326. Chakraborty P, Sabharwall P, Carroll MC (2016) A phase-field approach to model multi-axial and microstructure dependent fracture in nuclear grade graphite. J Nucl Mater 475:200-208

327. Gaston D, Newman C, Hansen G, Lebrun-Grandie D (2009) Moose: A parallel computational framework for coupled systems of nonlinear equations. Nucl Eng Des 239(10):1768-1778

328. Heister T, Wick $T$ (2020) pfm-cracks: A parallel-adaptive framework for phase-field fracture propagation. Softw Impacts $6: 100045$

329. Klinsmann M, Rosato D, Kamlah M, McMeeking RM (2015) An assessment of the phase field formulation for crack growth. Comput Methods Appl Mech Eng 294:313-330

330. Alnæs M, Blechta J, Hake J, Johansson A, Kehlet B, Logg A, Richardson C, Ring J, Rognes ME, Wells GN (2015) The fenics project version 1.5. Archive of Numerical Software, 3(100)

331. Ziaei-Rad V, Shen Y (2016) Massive parallelization of the phase field formulation for crack propagation with time adaptivity. Comput Methods Appl Mech Eng 312:224-253

332. Bourdin B (2019) bourdin/mef 90 0.1.0

333. Burke S, Ortner C, Süli E (2013) An adaptive finite element approximation of a generalized Ambrosio-Tortorelli functional M3AS, 23(9):1663-1697

334. Mang K, Walloth M, Wick T, Wollner W (2020) Mesh adaptivity for quasi-static phase-field fractures based on a residual-type a posteriori error estimator. GAMM-Mitteilungen 43(1):e202000003

335. Walloth M, Wollner W (2021) A posteriori estimator for the adaptive solution of a quasi-static fracture phase-field model with irreversibility constraints

336. Wick T (2016) Goal functional evaluations for phase-field fracture using pu-based dwr mesh adaptivity. Comput Mech 57(6):10171035

337. Artina M, Fornasier M, Micheletti S, Perotto S (2015) Anisotropic mesh adaptation for crack detection in brittle materials. SIAM J Sci Comput 37(4):B633-B659

338. Wick T (2021) Dual-weighted residual a posteriori error estimates for a penalized phase-field slit discontinuity problem. Comput Methods Appl Math 21(3):693-707

339. Andersson J, Mikayelyan H (2012) The asymptotics of the curvature of the free discontinuity set near the cracktip for the minimizers of the mumford-shah functional in the plain. arXiv preprint arXiv: 1204.5328

340. Bonnet A, David G (2001) Cracktip is a global Mumford-Shah minimizer. Société mathématique de France

341. Bellettini G, Coscia A (1994) Discrete approximation of a free discontinuity problem. Numer Funct Anal Optim 15(3-4):201224

342. Schröder J, Wick T, Reese S, Wriggers P, Müller R, Kollmannsberger S, Kästner M, Schwarz A, Igelbüscher M, Viebahn N, Bayat HR, Wulfinghoff S, Mang K, Rank E, Bog T, d'Angella D, Elhaddad M, Hennig P, Düster A, Garhuom W, Hubrich S, Walloth M, Wollner W, Kuhn C, Heister T (2021) A selection of benchmark 
problems in solid mechanics and applied mathematics. Arch Computat Methods Eng 28:713-751

343. Negri M (1999) The anisotropy introduced by the mesh in the finite element approximation of the mumford-shah functional. Numer Funct Anal Optim 20(9-10):957-982

344. Linse T, Hennig P, Kästner M, de Borst R (2017) A convergence study of phase-field models for brittle fracture. Eng Fract Mech 184:307-318

345. Badnava H, Msekh MA, Etemadi E, Rabczuk T (2018) An hadaptive thermo-mechanical phase field model for fracture. Finite Elem Anal Des 138:31-47

346. Patil R, Mishra B, Singh I (2018) An adaptive multiscale phase field method for brittle fracture. Comput Methods Appl Mech Eng 329:254-288

347. Wick T (2017) Coupling fluid-structure interaction with phasefield fracture: algorithmic details. In: Fluid-Structure Interaction: Modeling, Adaptive Discretization and Solvers (S. Frei, B. Holm, T. Richter, T. Wick, and H. Yang, eds.), Radon Series on Computational and Applied Mathematics, Walter de Gruyter, Berlin

348. Aldakheel F (2021) Simulation of fracture processes using globallocal approach and virtual elements. Habilitation thesis, Leibniz University Hannover. https://doi.org/10.15488/11367

349. Gerasimov T, Noii N, Allix O, De Lorenzis L (2018) A nonintrusive global/local approach applied to phase-field modeling of brittle fracture. Adv Model Simul Eng Sci 5:14

350. Aldakheel F, Noii N, Wick T, Wriggers P (2020) A global-local approach for hydraulic phase-field fracture in poroelastic media. accepted for publication in Computers and Mathematics with Applications (CAMWA)

351. Aldakheel F, Noii N, Wick T, Allix O, Wriggers P (2021) Multilevel global-local techniques for adaptive ductile phase-field fracture. Comput Methods Appl Mech Eng 387:114175

352. Gräser C, Kienle D, Sander O (2021) Truncated nonsmooth newton multigrid for phase-field brittle-fracture problems

353. Kristensen PK, Martinez-Paneda E (2020) Phase field fracture modelling using quasi-newton methods and a new adaptive step scheme. Theoret Appl Fract Mech 107:102446

354. Zhang X, Vignes C, Sloan SW, Sheng D (2017) Numerical evaluation of the phase-field model for brittle fracture with emphasis on the length scale. Comput Mech 59(5):737-752

355. Zhuang X, Zhou S, Sheng M, Li G (2020) On the hydraulic fracturing in naturally-layered porous media using the phase field method. Eng Geol 266:105306

356. Noll T, Kuhn C, Olesch D, Müller R (2019) 3d phase field simulations of ductile fracture," GAMM-Mitteilungen, p e202000008

357. Chukwudozie C, Bourdin B, Yoshioka K (2019) A variational phase-field model for hydraulic fracturing in porous media. Comput Methods Appl Mech Eng 347:957-982

358. Haslach HW Jr (2011) Maximum dissipation non-equilibrium thermodynamics and its geometric structure. Springer Science \& Business Media, Berlin

359. Ulmer H, Hofacker M, Miehe C (2013) Phase field modeling of brittle and ductile fracture. PAMM 13(1):533-536

360. Mauthe S, Miehe C (2015) Phase-field modeling of hydraulic fracture. PAMM 15(1):141-142

361. Miehe C, Teichtmeister S, Aldakheel F (2016) Phase-field modelling of ductile fracture: a variational gradient-extended plasticity-damage theory and its micromorphic regularization. Philos Trans R Soc A: Math, Phys Eng Sci 374(2066):20150170

362. Roy P, Pathrikar A, Deepu S, Roy D (2017) Peridynamics damage model through phase field theory. Int J Mech Sci 128:181-193

363. Farrahi GH, Javanbakht M, Jafarzadeh H (2018) On the phase field modeling of crack growth and analytical treatment on the parameters. Continuum Mechanics and Thermodynamics, pp 118
364. Alessi R, Marigo J-J, Maurini C, Vidoli S (2018) Coupling damage and plasticity for a phase-field regularisation of brittle, cohesive and ductile fracture: one-dimensional examples. Int J Mech Sci 149:559-576

365. Jeffrey R, Bunger A (2009) A detailed comparison of experimental and numerical data on hydraulic fracture height growth through stress contrasts. SPE J 14:413-422

366. Diehl P, Tabiai I, Baumann FW, Therriault D, Levesque M (2018) Long term availability of raw experimental data in experimental fracture mechanics. Eng Fract Mech 197:21-26

367. Devore JL (2011) Probability and statistics for engineering and the sciences. Cengage learning

368. Virtanen P, Gommers R, Oliphant TE, Haberland M, Reddy T, Cournapeau D, Burovski E, Peterson P, Weckesser W, Bright J, van der Walt SJ, Brett M, Wilson J, Jarrod Millman K, Mayorov N, Nelson ARJ, Jones E, Kern R, Larson E, Carey C, Polat İ, Feng Y, Moore EW, Vand erPlas J, Laxalde D, Perktold J, Cimrman R, Henriksen I, Quintero EA, Harris CR, Archibald AM, Ribeiro AH, Pedregosa F, van Mulbregt PS (2020) Contributors, Scipy 1.0: Fundamental algorithms for scientific computing in python. Nature Methods

369. Boyce BL, Kramer SL, Fang HE, Cordova TE, Neilsen MK, Dion K, Kaczmarowski AK, Karasz E, Xue L, Gross AJ et al (2014) The sandia fracture challenge: blind round robin predictions of ductile tearing. Int J Fract 186(1-2):5-68

370. Hu T, Talamini B, Stershic AJ, Tupek MR, Dolbow JE (2021) A variational phase-field model for ductile fracture with coalescence dissipation

371. Behzadinasab M, Foster JT (2019) The third sandia fracture challenge: peridynamic blind prediction of ductile fracture characterization in additively manufactured metal. Int J Fract 218(1):97-109

372. Kramer SL, Jones A, Mostafa A, Ravaji B, Tancogne-Dejean T, Roth CC, Bandpay MG, Pack K, Foster JT, Behzadinasab M et al (2019) The third sandia fracture challenge: predictions of ductile fracture in additively manufactured metal. Int J Fract 218(1-2):561

373. Behzadinasab M, Foster JT (2020) Revisiting the third sandia fracture challenge: a bond-associated, semi-lagrangian peridynamic approach to modeling large deformation and ductile fracture. Int J Fract 224:261-267

374. Dittmann M, Aldakheel F, Schulte J, Wriggers P, Hesch C (2018) Variational phase-field formulation of non-linear ductile fracture. Comput Methods Appl Mech Eng 342:71-94

375. Hocine NA, Abdelaziz MN, Mesmacque G (1998) Experimental and numerical investigation on single specimen methods of determination of $\mathrm{j}$ in rubber materials. Int J Fract 94(4):321-338

376. Talamini B, Mao Y, Anand L (2018) Progressive damage and rupture in polymers. J Mech Phys Solids 111:434-457

377. Behera D, Roy P, Madenci E (2020) Peridynamic correspondence model for finite elastic deformation and rupture in neo-hookean materials. Int J Non-Linear Mech 126:103564

378. Sundaram BM, Tippur HV (2018) Dynamic fracture of soda-lime glass: A full-field optical investigation of crack initiation, propagation and branching. J Mech Phys Solids 120:132-153

379. Mehrmashhadi J, Bahadori M, Bobaru F (2020) On validating peridynamic models and a phase-field model for dynamic brittle fracture in glass. Engineering Fracture Mechanics, p 107355

380. Ren B, Wu C (2018) A peridynamic model for damage prediction fiber-reinforced composite laminate. In: 15th International LSDYNA User Conference, $\mathrm{p} 10$

381. Dayal K, Bhattacharya K (2006) Kinetics of phase transformations in the peridynamic formulation of continuum mechanics. J Mech Phys Solids 54(9):1811-1842

382. Huang D, Lu G, Qiao P (2015) An improved peridynamic approach for quasi-static elastic deformation and brittle fracture analysis. Int J Mech Sci 94:111-122 
383. Mikata Y (2012) Analytical solutions of peristatic and peridynamic problems for a $1 \mathrm{~d}$ infinite rod. Int J Solids Struct 49(21):2887-2897

384. Zaccariotto M, Luongo F, Galvanetto U et al (2015) Examples of applications of the peridynamic theory to the solution of static equilibrium problems. Aeronaut J 119(1216):677-700

385. Buryachenko VA, Wanji C, Shengqi Y (2015) Effective thermoelastic properties of heterogeneous thermoperistatic bar of random structure. International Journal for Multiscale Computational Engineering, 13(1)

386. Hofacker M, Miehe C (2013) A phase field model of dynamic fracture: Robust field updates for the analysis of complex crack patterns. Int J Numer Meth Eng 93(3):276-301

387. Noll T, Kuhn C, Müller R (2017) A monolithic solution scheme for a phase field model of ductile fracture. PAMM 17(1):75-78

388. Tanné E, Li T, Bourdin B, Marigo J-J, Maurini C (2018) Crack nucleation in variational phase-field models of brittle fracture. $\mathrm{J}$ Mech Phys Solids 110:80-99

389. Sneddon IN, Lowengrub M (1969) Crack problems in the classical theory of elasticity. SIAM series in Applied Mathematics. Wiley, Philadelphia

390. Goswami S, Anitescu C, Chakraborty S, Rabczuk T (2020) Transfer learning enhanced physics informed neural network for phase-field modeling of fracture. Theoret Appl Fract Mech 106:102447

391. Nguyen CT, Oterkus S, Oterkus E (2021) A physics-guided machine learning model for two-dimensional structures based on ordinary state-based peridynamics. Theoret Appl Fract Mech $112: 102872$

392. Mandal TK (2021) Phase field fracture modelling of solids: dynamics, anisotropy, and multi-physics. Dissertation, Monash University, Australia

393. Bobaru F, Zhang G (2015) Why do cracks branch? a peridynamic investigation of dyanmic brittle fracture. Int J Fract 196(1):59-98

394. Silling S, Weckner O, Ascari E, Bobaru F (2010) Crack nucleation in a peridynamic solid. Int J Fract 162:219-227

395. Tupek MR, Rimoli JJ, Radovitzky R (2013) An approach for incorporating classical continuum damage models in state-based peridynamics. Comput Methods Appl Mech Eng 263:42-57

396. Bobaru F, Duangpanya M (2010) The peridynamic formulation for transient heat conduction. Int J Heat Mass Transf 53(1920):4047-4059

397. Oterkus S, Madenci E, Agwai A (2014) Peridynamic thermal diffusion. J Comput Phys 265:71-96

398. Oterkus S, Madenci E, Agwai A (2014) Fully coupled peridynamic thermomechanics. J Mech Phys Solids 64:1-23

399. Chen Z, Bobaru F (2015) Selecting the kernel in a peridynamic formulation: a study for transient heat diffusion. Comput Phys Commun 197:51-60

400. Liao Y, Liu L, Liu Q, Lai X, Assefa M, Liu J (2017) Peridynamic simulation of transient heat conduction problems in functionally gradient materials with cracks. J Therm Stresses 40(12):14841501

401. Diyaroglu C, Oterkus S, Oterkus E, Madenci E, Han S, Hwang Y (2017) Peridynamic wetness approach for moisture concentration analysis in electronic packages. Microelectron Reliab 70:103-111

402. Tao Y, Tian X, Du Q (2017) Nonlocal diffusion and peridynamic models with neumann type constraints and their numerical approximations. Appl Math Comput 305:282-298

403. Gu X, Zhang Q, Madenci E (2019) Refined bond-based peridynamics for thermal diffusion. Engineering Computations

404. Ouchi H, Katiyar A, York J, Foster JT, Sharma MM (2015) A fully coupled porous flow and geomechanics model for fluid driven cracks: a peridynamics approach. Comput Mech 55(3):561-576

405. Wang Y, Zhou X, Xu X (2016) Numerical simulation of propagation and coalescence of flaws in rock materials under compressive loads using the extended non-ordinary state-based peridynamics. Eng Fract Mech 163:248-273

406. Zhou X, Shou Y (2016) Numerical simulation of failure of rocklike material subjected to compressive loads using improved peridynamic method. Int J Geomech 17(3):04016086

407. Zhou X-P, Gu X-B, Wang Y-T (2015) Numerical simulations of propagation, bifurcation and coalescence of cracks in rocks. Int $\mathrm{J}$ Rock Mech Mining Sci 80:241-254

408. Ren B, Fan H, Bergel GL, Regueiro RA, Lai X, Li S (2015) A peridynamics-sph coupling approach to simulate soil fragmentation induced by shock waves. Comput Mech 55(2):287-302

409. Fan H, Li S (2017) A peridynamics-sph modeling and simulation of blast fragmentation of soil under buried explosive loads. Comput Methods Appl Mech Eng 318:349-381

410. Nadimi S, Miscovic I, McLennan J (2016) A 3d peridynamic simulation of hydraulic fracture process in a heterogeneous medium. J Petrol Sci Eng 145:444-452

411. Wu F, Li S, Duan Q, Li X (2016) Application of the method of peridynamics to the simulation of hydraulic fracturing process. International Conference on Discrete Element Methods. Springer, Berlin, pp 561-569

412. Panchadhara R, Gordon PA, Parks ML (2017) Modeling propellant-based stimulation of a borehole with peridynamics. Int J Rock Mech Min Sci 93:330-343

413. Lai X, Ren B, Fan H, Li S, Wu C, Regueiro RA, Liu L (2015) Peridynamics simulations of geomaterial fragmentation by impulse loads. Int J Numer Anal Meth Geomech 39(12):1304-1330

414. Yan F, Feng X-T, Pan P-Z, Li S-J (2014) A continuousdiscontinuous cellular automaton method for cracks growth and coalescence in brittle material. Acta Mech Sin 30(1):73-83

415. Chen Z, Bobaru F (2015) Peridynamic modeling of pitting corrosion damage. J Mech Phys Solids 78:352-381

416. Rokkam S, Phan N, Gunzburger M, Shanbhag S, Goel K (2018) "Meshless peridynamics method for modeling corrosion crack propagation," In: 6th International Conference on Crack Paths (CP 2018)(Verona, Italy). http://www.cp2018.unipr.it

417. Jafarzadeh S, Chen Z, Bobaru F (2018) Peridynamic modeling of intergranular corrosion damage. J Electrochem Soc 165(7):C362C374

418. Miehe C, Mauthe S, Teichtmeister S (2015) Minimization principles for the coupled problem of darcy-biot-type fluid transport in porous media linked to phase field modeling of fracture. J Mech Phys Solids 82:186-217

419. Miehe C, Mauthe S (2016) Phase field modeling of fracture in multi-physics problems. part iii. crack driving forces in hydroporo-elasticity and hydraulic fracturing of fluid-saturated porous media. Comput Methods Appl Mech Eng 304:619-655

420. Wilson ZA, Landis CM (2016) Phase-field modeling of hydraulic fracture. J Mech Phys Solids 96:264-290

421. Heider Y, Markert B (2017) A phase-field modeling approach of hydraulic fracture in saturated porous media. Mech Res Commun 80:38-46

422. Heider Y, Reiche S, Siebert P, Markert B (2018) Modeling of hydraulic fracturing using a porous-media phase-field approach with reference to experimental data. Eng Fract Mech 202:116-134

423. Ehlers W, Luo C (2017) A phase-field approach embedded in the theory of porous media for the description of dynamic hydraulic fracturing. Comput Methods Appl Mech Eng 315:348-368

424. Zhou S, Zhuang X, Rabczuk T (2019) Phase-field modeling of fluid-driven dynamic cracking in porous media. Comput Methods Appl Mech Eng 350:169-198

425. van Duijn C, Mikelić A, Wick T (2019) A monolithic phase-field model of a fluid-driven fracture in a nonlinear poroelastic medium. Math Mech Solids 24(5):1530-1555 
426. Noii N, Khodadadian A, Wick T (2021) Bayesian inversion for anisotropic hydraulic phase-field fracture. Comput Methods Appl Mech Eng 386:114118

427. Yi L-P, Waisman H, Yang Z-Z, Li X-G (2020) A consistent phase field model for hydraulic fracture propagation in poroelastic media. Comput Methods Appl Mech Eng 372:113396

428. Heider Y (2021) Multi-field and multi-scale computational fracture mechanics and machine-learning material modeling. Habilitation thesis, Rheinisch-Westfälische Technische Hochschule Aachen

429. Mandal TK, Nguyen VP, Wu J-Y, Nguyen-Thanh C, de Vaucorbeil A (2021) Fracture of thermo-elastic solids: Phase-field modeling and new results with an efficient monolithic solver. Comput Methods Appl Mech Eng 376:113648

430. You T, Waisman H, Zhu Q-Z (2021) Brittle-ductile failure transition in geomaterials modeled by a modified phase-field method with a varying damage-driving energy coefficient. Int $\mathrm{J}$ Plast 136:102836

431. Wick T (2016) Coupling fluid-structure interaction with phasefield fracture. J Comput Phys 327:67-96

432. Freund L (1989) Dynamic fracture mechanics. Cambridge University Press, Cambridge

433. Dal Maso G, Lazzaroni G, Nardini L (2016) Existence and uniqueness of dynamic evolutions for a peeling test in dimension one. $\mathrm{J}$ Differ Equ 261:4897-4923

434. Lazzaroni G, Nardini L (2018) On the quasistatic limit of dynamic evolutions for a peeling test in dimension one. J Nonlinear Sci 28:268-304

435. Wang H, Tian $\mathrm{H}$ (2012) A fast galerkin method with efficient matrix assembly and storage for a peridynamic model. J Comput Phys 231(23):7730-7738

436. Prakash N, Stewart RJ (2020) A multi-threaded method to assemble a sparse stiffness matrix for quasi-static solutions of linearized bond-based peridynamics. Journal of Peridynamics and Nonlocal Modeling, pp 1-35

437. Cassell A, Hobbs R (1976) Numerical stability of dynamic relaxation analysis of non-linear structures. Int J Numer Meth Eng 10(6):1407-1410

438. Topping B, Khan A (1994) Parallel computation schemes for dynamic relaxation. Eng Comput 11(6):513-548

439. Kilic B, Madenci E (2010) An adaptive dynamic relaxation method for quasi-static simulations using the peridynamic theory. Theoret Appl Fract Mech 53(3):194-204

440. Shiihara Y, Tanaka S, Yoshikawa N (2019) Fast quasi-implicit nosb peridynamic simulation based on fire algorithm. Mech Eng J 6(3):18-00363

441. Bitzek E, Koskinen P, Gähler F, Moseler M, Gumbsch P (2006) Structural relaxation made simple. Phys Rev Lett 97(17):170201

442. Diehl P, Lipton R (2021) Quasistatic fracture using nonlinernonlocal elastostatics with explicit tangent stiffness matrix

443. Jafarzadeh S, Wang L, Larios A, Bobaru F (2021) A fast convolution-based method for peridynamic transient diffusion in arbitrary domains. Comput Methods Appl Mech Eng 375:113633

444. Jafarzadeh S, Mousavi F, Larios A, Bobaru F (2021) A general and fast convolution-based method for peridynamics: applications to elasticity and brittle fracture

445. Saad Y, Schultz MH (1986) Gmres: A generalized minimal residual algorithm for solving nonsymmetric linear systems. SIAM J Sci Stat Comput 7(3):856-869

446. Arnoldi W (1951) The principle of minimized iteration in the solution of the matrix eigenvafue problem. Quart Appl Math 9:1729

447. Saad Y (1981) Krylov subspace methods for solving large unsymmetric linear systems. Math Comput 37(155):105-126

448. Nguyen N-H, Nguyen VP, Wu J-Y, Le T-H-H, Ding Y et al (2019) Mesh-based and meshfree reduced order phase-field mod- els for brittle fracture: One dimensional problems. Materials 12(11): 1858

449. Kerfriden P, Goury O, Rabczuk T, Bordas SP-A (2013) A partitioned model order reduction approach to rationalise computational expenses in nonlinear fracture mechanics. Comput Methods Appl Mech Eng 256:169-188

450. Kane C, Marsden JE, Ortiz M (1999) Symplectic-energymomentum preserving variational integrators. J Math Phys 40(7):3353-3371

451. Shao Y, Duan Q, Qiu S (2021) Adaptive analysis for phase-field model of brittle fracture of functionally graded materials. Engineering Fracture Mechanics, p 107783

452. Geelen R, Plews J, Tupek M, Dolbow J (2020) An extended/generalized phase-field finite element method for crack growth with global-local enrichment. Int J Numer Meth Eng 121(11):2534-2557

453. Ouchi H, Katiyar A, Foster JT, Sharma MM et al (2017) A peridynamics model for the propagation of hydraulic fractures in naturally fractured reservoirs. SPE J 22(04):1-082

454. Ni T, Zaccariotto M, Zhu Q-Z, Galvanetto U (2019) Coupling of fem and ordinary state-based peridynamics for brittle failure analysis in 3d. Mechanics of Advanced Materials and Structures, pp 1-16

455. Bobaru F, Silling SA (2004) Peridynamic 3d models of nanofiber networks and carbon nanotube-reinforced composites. In: AIP Conference Proceedings, vol. 712, pp. 1565-1570, American Institute of Physics

456. Jafarzadeh S, Chen Z, Zhao J, Bobaru F (2019) Pitting, lacy covers, and pit merger in stainless steel: $3 \mathrm{~d}$ peridynamic models. Corros Sci 150:17-31

457. Bobaru F, Ha YD, Hu W (2012) Damage progression from impact in layered glass modeled with peridynamics. Cent Eur J Eng 2(4):551-561

458. Hu W, Wang Y, Yu J, Yen C-F, Bobaru F (2013) Impact damage on a thin glass plate with a thin polycarbonate backing. Int J Impact Eng 62:152-165

459. Breitenfeld M (2014) Quasi-static non-ordinary state-based peridynamics for the modeling of 3D fracture. Ph.D thesis, University of Illinois at Urbana-Champaign

460. Liu W, Hong J-W (2012) Discretized peridynamics for brittle and ductile solids. Int J Numer Meth Eng 89(8):1028-1046

461. Dally T, Weinberg K (2017) The phase-field approach as a tool for experimental validations in fracture mechanics. Continuum Mech Thermodyn 29(4):947-956

462. Noii N, Wick T (2019) A phase-field description for pressurized and non-isothermal propagating fractures. Comput Methods Appl Mech Eng 351:860-890

463. Weinberg K, Dally T, Schuß S, Werner M, Bilgen C (2016) Modeling and numerical simulation of crack growth and damage with a phase field approach. GAMM-Mitteilungen 39(1):55-77

464. Agwai A, Guven I, Madenci E (2011) Predicting crack propagation with peridynamics: a comparative study. Int J Fract 171(1):65

465. Ha YD, Bobaru F (2010) Studies of dynamic crack propagation and crack branching with peridynamics. Int J Fract 162(1-2):229244

466. Agrawal V, Dayal K (2017) Dependence of equilibrium griffith surface energy on crack speed in phase-field models for fracture coupled to elastodynamics. Int J Fract 207(2):243-249

467. Yoshioka K, Naumov D, Kolditz O (2020) On crack opening computation in variational phase-field models for fracture. Comput Methods Appl Mech Eng 369:113210

468. Sato S, Awaji H, Kawamata K, Kurumada A, Oku T (1987) Fracture criteria of reactor graphite under multiaxial stesses. Nucl Eng Des 103(3):291-300

469. Cristiano A, Marcellan A, Long R, Hui C-Y, Stolk J, Creton C (2010) An experimental investigation of fracture by cavitation of 
model elastomeric networks. J Polym Sci, Part B: Polym Phys 48(13):1409-1422

470. Gómez F, Elices M, Berto F, Lazzarin P (2009) Fracture of vnotched specimens under mixed mode (i+ ii) loading in brittle materials. Int J Fract 159(2):121-135

471. Kimoto H, Usami S, Miyata H (1985) Flaw size dependence in fracture stress of glass and polycrystalline ceramics. Trans Jpn Soc Mech Eng (Ser A) 51(471):2482-2488

472. Spetz A, Denzer R, Tudisco E, Dahlblom O (2020) Phase-field fracture modelling of crack nucleation and propagation in porous rock. Int J Fract 224:31-46

473. Silling SA, Weckner O, Askari E, Bobaru F (2010) Crack nucleation in a peridynamic solid. Int J Fract 162(1-2):219-227

474. Littlewood DJ (2011) A nonlocal approach to modeling crack nucleation in aa 7075-t651. ASME Int Mech Eng Congr Expos 54945:567-576

475. Niazi S, Chen Z, Bobaru F (2021) Crack nucleation in brittle and quasi-brittle materials: a peridynamic analysis. Theoret Appl Fract Mech 112:102855

476. Mang K (2021) Phase-field fracture modeling, numerical solution, and simulations for compressible and incompressible solids. Ph.D thesis, Leibniz University Hannover

477. Bang D, Madenci E (2017) Peridynamic modeling of hyperelastic membrane deformation. Journal of Engineering Materials and Technology, 139(3)

478. Waxman R, Guven I (2020) Implementation of a neo-hookean material model in state-based peridynamics to represent nylon bead behavior during high-speed impact. In: AIAA Scitech 2020 Forum, p 0725

479. Ogden RW (1997) Non-linear elastic deformations. Courier Corporation, North Chelmsford

480. Rivlin R (1948) Large elastic deformations of isotropic materials iv. further developments of the general theory. Philos Trans R Soc London. Ser A, Math Phys Sci 241(835):379-397

481. Ahadi A, Melin S (2018) Capturing nanoscale effects by peridynamics. Mech Adv Mater Struct 25(13):1115-1120

482. Bitzek E, Kermode JR, Gumbsch P (2015) Atomistic aspects of fracture. Int J Fract 191:13-30

483. Patil SP, Heider Y, Padilla CAH, Cruz-Chú ER, Markert B (2016) A comparative molecular dynamics-phase-field modeling approach to brittle fracture. Comput Methods Appl Mech Eng 312:117-129

484. Buehler MJ (2008) Atomistic modeling of materials failure. Springer, Berlin

485. Du Q (2016) Nonlocal calculus of variations and well-posedness of peridynamics. In: Handbook of peridynamic modeling, pp 101124, Chapman and Hall/CRC

486. Gu X, Madenci E, Zhang Q (2018) Revisit of non-ordinary statebased peridynamics. Eng Fract Mech 190:31-52

487. Madenci E, Dorduncu M, Barut A, Phan N (2018) A state-based peridynamic analysis in a finite element framework. Eng Fract Mech 195:104-128

488. Madenci E, Dorduncu M, Barut A, Phan N (2018) Weak form of peridynamics for nonlocal essential and natural boundary conditions. Comput Methods Appl Mech Eng 337:598-631

489. Prudhomme S, Diehl P (2020) On the treatment of boundary conditions for bond-based peridynamic models. Comput Methods Appl Mech Eng 372:113391

490. Mei T, Zhao J, Liu Z, Peng X, Chen Z, Bobaru F (2021) The role of boundary conditions on convergence properties of peridynamic model for transient heat transfer. J Sci Comput 87(2):1-22

491. D'Elia M, Li X, Seleson P, Tian X, Yu Y (2019) A review of localto-nonlocal coupling methods in nonlocal diffusion and nonlocal mechanics. arXiv preprint arXiv: 1912.06668
492. Silling SA (2017) Stability of peridynamic correspondence material models and their particle discretizations. Comput Methods Appl Mech Eng 322:42-57

493. Foster JT, Xu X (2018) A generalized, ordinary, finite deformation constitutive correspondence model for peridynamics. Int J Solids Struct 141:245-253

494. Deepak B, Pranesh R, Erdogan M (2020) An approach for incorporating classical continuum damage models in state-based peridynamics. J Non-Linear Mech 126:103564

495. Chowdhury SR, Roy P, Roy D, Reddy J (2019) A modified peridynamics correspondence principle: Removal of zero-energy deformation and other implications. Computer Methods in Applied Mechanics and Engineering

496. Silling SA (2011) A coarsening method for linear peridynamics. International Journal for Multiscale Computational Engineering, 9(6)

497. Delorme R, Diehl P, Tabiai I, Lebel LL, Lévesque M (2020) Extracting constitutive mechanical parameters in linear elasticity using the virtual fields method within the ordinary state-based peridynamic framework. Journal of Peridynamics and Nonlocal Modeling, pp 1-25

498. Le Q, Bobaru F (2018) Surface corrections for peridynamic models in elasticity and fracture. Comput Mech 61(4):499-518

499. Madenci E, Oterkus E (2014) Coupling of the peridynamic theory and finite element method. Peridynamic theory and its applications. Springer, Berlin, pp 191-202

500. Shen S, Yang Z, Han F, Cui J, Zhang J (2021) Peridynamic modeling with energy-based surface correction for fracture simulation of random porous materials. Theoret Appl Fract Mech 114:102987

501. Gerstle W, Sau N, Silling S (2005) Peridynamic modeling of plain and reinforced concrete structures. In: Proceedings of 18th International Conference on Structural Mechanics in Reactor Technology

502. Tan Y, Liu Q, Zhang L, Liu L, Lai X (2020) Peridynamics model with surface correction near insulated cracks for transient heat conduction in functionally graded materials. Materials 13(6):1340

503. Florin B, Youn DH (2011) Adaptive refinement and multiscale modeling in 2d peridynamics. J Multiscale Comput Eng 9:635659

504. Yunzhe T, Xiaochuan T, Qiang D (2019) Nonlocal models with heterogeneous localization and their application to seamless localnonlocal coupling. Multiscale Model Simul 17:1052-1075

505. Wildman RA, Gazonas GA (2014) A finite difference-augmented peridynamics methodfor reducing wave dispersion. Int $\mathrm{J}$ Fract 190:39-52

506. Yolum U, Taştan A, Güler MA (2016) A peridynamic model for ductile fracture of moderately thick plates. Procedia Struct Integr 2:3713-3720

507. Conradie J, Becker T, Turner D (2019) Peridynamic approach to predict ductile and mixed-mode failure. R D J 35:1-8

508. Behzadinasab M (2019) Peridynamic modeling of large deformation and ductile fracture. Ph.D thesis, UT Austin

509. Behzadinasab M, Foster JT (2020) A semi-lagrangian constitutive correspondence framework for peridynamics. J Mech Phys Solids 137:103862

510. Chen Z, Niazi S, Bobaru F (2019) A peridynamic model for brittle damage and fracture in porous materials. Int J Rock Mech Min Sci 122:104059

511. Freimanis A, Paeglitis A (2017) Mesh sensitivity in peridynamic quasi-static simulations. Procedia Eng 172:284-291

512. Wang F, Ma Y, Guo Y, Huang W (2019) Studies on quasi-static and fatigue crack propagation behaviours in friction stir welded joints using peridynamic theory. Advances in Materials Science and Engineering, 2019

513. Biner S, Hu SY (2009) Simulation of damage evolution in composites: a phase-field model. Acta Mater 57(7):2088-2097 
514. Doan DH, Bui TQ, Duc ND, Fushinobu K (2016) Hybrid phase field simulation of dynamic crack propagation in functionally graded glass-filled epoxy. Compos B Eng 99:266-276

515. Feng D-C, Wu J-Y (2018) Phase-field regularized cohesive zone model (czm) and size effect of concrete. Eng Fract Mech 197:6679

516. Yang Z-J, Li B-B, Wu J-Y (2019) X-ray computed tomography images based phase-field modeling of mesoscopic failure in concrete. Eng Fract Mech 208:151-170

517. Nguyen VP, Wu J-Y (2018) Modeling dynamic fracture of solids with a phase-field regularized cohesive zone model. Comput Methods Appl Mech Eng 340:1000-1022

518. Santillán D, Mosquera JC, Cueto-Felgueroso L (2017) Phase-field model for brittle fracture. validation with experimental results and extension to dam engineering problems. Eng Fract Mech 178:109-125

519. Bourdin B (1999) Image segmentation with a finite element method. Math Model Numer Anal 33(2):229-244

520. Feng X, Prohl A (2003) Numerical analysis of the Allen-Cahn equation and approximation for mean curvature flows. Numer Math 94:33-65
521. Feng X, Prohl A (2004) Analysis of a fully discrete finite element method for the phase field model and approximation of its sharp interface limits. Math Comp 73:541-567

522. Kolditz L (2021) $\Gamma$-convergence of a pressurized phase-field fracture model. Bachelor's thesis, Leibniz Universität Hannover

523. Kolditz L, Mang K (2021) On the relation of Gamma-convergence parameters for pressure-driven quasi-static phase-field fracture. Examples and Counterexamples, in review

524. Jodlbauer D (2021) Parallel multigrid solvers for nonlinear coupled field problems. Ph.D thesis, Johannes Kepler University Linz

525. Hrennikoff A (1941) Solution of problems of elasticity by the framework method

526. Courant R et al., (1994) Variational methods for the solution of problems of equilibrium and vibrations. Lecture notes in pure and applied mathematics, pp 1-1

527. Zienkiewicz OC, Taylor RL, Nithiarasu P, Zhu J (1977) The finite element method, vol 3. McGraw-hill, London

Publisher's Note Springer Nature remains neutral with regard to jurisdictional claims in published maps and institutional affiliations. 